\title{
Implications of Yukawa unification for the Higgs sector in supersymmetric grand-unified models
}

\author{
Paul Langacker and Nir Polonsky \\ Department of Physics, University of Pennsylvania, Philadelphia, Pennsylvania, 19104, USA
}

(February 1994, UPR-0594T)

\begin{abstract}
The $S U(5)$ unification-scale relation $h_{b}=h_{\tau}$ between the $b$-quark and $\tau$ lepton Yukawa couplings severely constrains $\tan \beta$ and the $t$-quark mass (even more so if $h_{t}=h_{b}=h_{\tau}$ holds) in supersymmetric models. We examine the implications of these constraints for the Higgs sector assuming universal soft breaking terms, and emphasize that both of these relations impose unique characteristics in terms of symmetries and of the spectrum. We further study the $\tan \beta \approx 1$ scenario, which is suggested by $h_{b}=h_{\tau}$, and, in particular, the loop-induced mass of the light Higgs boson. We compare the effective potential and renormalization group methods and stress the two-loop ambiguities in the calculation of the mass. These and a large enhancement to the loop correction due to $t$-scalar left-right mixing considerably weaken the upper bound on the mass of the light Higgs boson that has been reported. Nevertheless, we find that for this scenario the Higgs boson is probably lighter than $110 \mathrm{GeV}$, and typically lighter than $100 \mathrm{GeV}$. Thus, it is in the mass range that may be relevant for LEPII. Our numerical results are presented in a self-contained manner in section $\square$. In separate appendices we discuss the global symmetries of the Higgs potential, the issue of false (color-breaking) vacua, which may be important for $\tan \beta \approx 1$, two-loop calculations, and the effect of an additional Higgs singlet. We show that the approximate constraints that are often used to eliminate color-breaking vacua are not always relevant.
\end{abstract}

PACS numbers: 12.10.Dm, 11.30.Pb, 14.80.Gt, 14.80.Ly 


\section{INTRODUCTION}

Supersymmetric grand-unified theories [1.2] (SGUT), though far from being proved, are an attractive framework on both observational and theoretical grounds. (Their relation to string theory remains at present poorly understood.) In the minimal models, which we assume hereafter, there is a grand desert between the weak and the unification scales $\left(M_{Z} \lesssim Q \lesssim \mathrm{TeV}\right.$ and $M_{G} \approx 10^{16}-10^{17} \mathrm{GeV}$, respectively). At the unification point $M_{G}$

$$
\alpha_{1}\left(M_{G}\right)=\alpha_{2}\left(M_{G}\right)=\alpha_{3}\left(M_{G}\right) \equiv \alpha_{G}
$$

(where $\alpha_{1}=\frac{5}{3} \alpha_{Y}$ and $\alpha_{G} \approx 0.04$ ), and, depending on the grand unifying group representations of the Higgs superfields that couple to matter, certain relations between the standard model Yukawa couplings hold. In the minimal models based on $S U(5), S O(10)$, and $E_{6}$ these representations are the fundamental ones, and one obtains [3]

$$
h_{\tau}\left(M_{G}\right)=h_{b}\left(M_{G}\right) .
$$

For some models based on $S O(10)$ (or larger groups) one has the stronger prediction

$$
h_{\tau}\left(M_{G}\right)=h_{b}\left(M_{G}\right)=h_{t}\left(M_{G}\right)
$$

but this only holds if one makes the additional assumption that the masses are generated by a single complex Higgs 10-plet. ( $h_{\tau, b, t}$ are the SM Yukawa couplings of the $\tau, b, t$-superfields, respectively.) One usually assumes that some perturbation modifies the coupling or the masses of the two light families where, in principle, similar relations should, but do not, hold . (See also Appendix D.) Also, such relations often do not hold in superstring unified models.

At the weak-scale the particle content is that of the minimal supersymmetric standard model (MSSM), i.e., that of a two Higgs doublet model but with each bosonic (fermionic) degree of freedom complemented by a fermionic (bosonic) one. In particular, the Higgs sector contains three Goldstone bosons and five physical degrees of freedom - two CP even $\left(h^{0}\right.$ and $\left.H^{0}\right)$ and one CP odd $\left(A^{0}\right)$ neutral, and one complex charged $\left(H^{+}\right)$Higgs bosons. The symmetries and spectrum of the Higgs scalars are strongly affected by the above GUT assumptions, and are the subject of our present study. However, a review of previous results in SGUT's, which establish the base for our present work, is appropriate.

The consistency of (11) with the data was pointed out recently in Ref. [4] and that of (2) and (3) in Ref. [5]. Relation (11), however, holds only if there is exactly one pair of Higgs doublets (but any number of SM singlets is allowed). We previously investigated the status of coupling constant unification in great detail [6] where we considered non-trivial matching conditions? that perturb relation (1) in a model dependent way. We concluded

\footnotetext{
${ }^{1}$ Since the Yukawa couplings corresponding to the third family are much larger than those of the two light families, relation (2) [or (3)] will be only slightly affected.

${ }^{2}$ Heavy degrees of freedom (which are model dependent) are integrated out of the effective theory
} 
that no significant constraints on the MSSM parameters can be deduced just by that relation. However, we find $]^{3}$ that (1) predicts for the strong coupling [using $\alpha\left(M_{Z}\right)^{-1}=127.9 \pm 0.1$ [7] and $s^{2}\left(M_{Z}\right)=0.2324 \pm 0.0003$ (for $m_{t}^{\text {pole }}=143 \mathrm{GeV}$ ) for the $(\overline{\mathrm{MS}})$ fine-structure constant and weak angle, respectively]

$$
\alpha_{s}\left(M_{Z}\right)=0.125 \pm 0.001 \pm 0.008+H_{\alpha_{s}}+3.2 \times 10^{-7} \mathrm{GeV}^{-2}\left[\left(m_{t}^{\text {pole }}\right)^{2}-(143 \mathrm{GeV})^{2}\right]
$$

where the $\pm 0.008( \pm 0.001)$ uncertainty in (4) is theoretical (due to the input parameter error bars). The former is due to the unknown values of the matching conditions (i.e., threshold and nonrenormalizable-operator effects) mentioned above. The function $H_{\alpha_{s}}$ is a correction from Yukawa interactions. It is negative but negligible, unless some Yukawa couplings are $\gtrsim 1$, for which we obtain $H_{\alpha_{s}} \approx-(0.001-0.003)$. The quadratic $m_{t}^{\text {pole }}$ dependence is induced by the correlation between the weak angle extracted from the data and the $t$-quark pole mass, $m_{t}^{\text {pole }}$. Thus, typically one predicts $\alpha_{s}\left(M_{Z}\right) \gtrsim 0.12$ with a significant dependence on $m_{t}^{\text {pole }}$. This observation is of importance when simultaneously considering (11) and (2) [or (3)] to predict the $b$-quark ( $\overline{\mathrm{MS}}$ running) mass $m_{b}$ [and/or the $t$-quark mass, if using (3)] in terms of the $\tau$-lepton mass. We find [using, e.g., Eq. (田) but with a more careful treatment of theoretical uncertainties to the $b$-quark mass prediction] that only a small region in the $\tan \beta-m_{t}^{\text {pole }}$ plane is allowed by the experimental range of $m_{b}$ [8], where $\tan \beta=\nu_{\text {up }} / \nu_{\text {down }}$ is the ratio of the two Higgs doublet expectation values. This holds even when non-trivial matching conditions at both scales are considered.

It is customary to assume that the MSSM spectrum is given to a good approximation near $M_{G}$ by a small number of universal supersymmetry-breaking soft parameters; i.e., a common scalar mass $m_{0}$, trilinear and bilinear (dimension-one) couplings $A_{0}$ and $B_{0}$, and a common gaugino mass $M_{\frac{1}{2}}$; in addition to a supersymmetric Higgs mass (i.e., the Higgsino mass), $\mu_{0}$ [2]. We will adopt these assumptions below. (For an alternative scenario, see, for example, Ref. [9].) However, our conclusions so far are largely (i.e., except for the the details of the matching conditions) independent of those assumptions and hold in any SGUT scenario with an approximate grand-desert in which the MSSM $\beta$-functions are valid. The universality assumption just strengthens the constraints on the $\tan \beta-m_{t}^{\text {pole }}$ plane; i.e., assuming universal initial conditions and requiring that the weak-scale MSSM (one-loop improved) scalar potential is consistent with a broken $S U(2) \times U(1)$ symmetry, we have to further constrain $\tan \beta \geq 1$ and $h_{t} \geq h_{b}$. We then find two allowed regions,

1. $\tan \beta=1+\Delta_{\beta}, 140 \lesssim m_{t}^{\text {pole }}(\lesssim 200) \mathrm{GeV}, h_{t} \approx 1 \gg h_{b}$;

2. $\tan \beta \approx 50 \pm 10,170 \pm 10 \lesssim m_{t}^{\text {pole }}(\lesssim 200) \mathrm{GeV}, h_{t} \gtrsim h_{b} \approx 1$.

at $M_{G}$ and some modification to the naive boundary conditions in (11) and (2) [or (3)] is required, in general. The appropriate matching functions may also include corrections from nonrenormalizable operators since $M_{G}$ is not too far from the Planck scale. Similarly, integrating out the MSSM new particle spectrum will correct the boundary conditions at $M_{Z}$.

${ }^{3}$ Eq. (四) updates the results in [6] for the effect of more recent precision electroweak data. 
The first of these solutions is consistent only with $h_{b}=h_{\tau}$, and has $\Delta_{\beta} \lesssim 1$ for $m_{t}^{\text {pole }} \lesssim 190$ $\mathrm{GeV}$. A part of the region which is described by the second solution is consistent with the stronger prediction $h_{\tau}=h_{b}=h_{t}$. A third solution with $3 \lesssim \tan \beta \lesssim 40$ exists for $m_{t}^{\text {pole }} \approx 215 \pm 10 \mathrm{GeV}$, which is the upper bound on $m_{t}^{\text {pole }}$ if the model is to stay perturbative up to $M_{G}$. However, such large values for $m_{t}$ are inconsistent with precision electroweak data. The allowed regions are illustrated in Fig. 1.

These results are in good agreement with those of Ref. [10 14], with any small differences due to different treatments of matching conditions and of the coupling constant unification condition. Arbitrarily relaxing both condition (1) and (2) independently [e.g., $\alpha_{s}\left(M_{Z}\right)=$ 0.11 and $h_{b}\left(M_{G}\right)=0.85 h_{\tau}\left(M_{G}\right)$ ] could invalidate our conclusions. However, the corrections to (11) and (2) are strongly correlated, and when treated as such the strong constraints on the $\tan \beta-m_{t}^{\text {pole }}$ plane hold 1 . In particular, the $m_{b} / m_{\tau}$ ratio is relatively insensitive to the details of the decoupling of the low-scale spectrum [8], and our conclusions would not be significantly modified if those thresholds were treated differently than in our (semianalytic) approximation (where we used six effective mass parameters). Let us stress that any treatment of theoretical uncertainties would be lacking unless the potentially important corrections at the high scale are accounted for in one form or another. These issues are summarized in Ref. [15,16, where the second reference updates some of the results of Ref. [6]:8]. Here, relevant results are again updated using a preliminary analysis of most recent precision data.

A two-parameter fit [allowing the light (SM-like) Higgs boson mass, $m_{h^{0}}$, to vary from $50-150 \mathrm{GeV}$ with a central value $m_{h^{0}}=M_{Z}$ ] to all $Z, W$, and neutral-current data yields

$$
\begin{gathered}
s^{2}\left(M_{Z}\right)=0.2324 \pm 0.0003-0.92 \times 10^{-7} \mathrm{GeV}^{-2}\left[\left(m_{t}^{\text {pole }}\right)^{2}-(143 \mathrm{GeV})^{2}\right] \\
m_{t}^{\text {pole }}=143_{-19}^{+17}+12.5 \ln \frac{m_{h^{0}}}{M_{Z}} \mathrm{GeV}
\end{gathered}
$$

which in turn implies $\tan \beta \lesssim 1.3$ (at one s.d.). Eqs. (5) and (6) update Ref. [17] where $m_{t}^{\text {pole }}=134_{-28}^{+23}+12.5 \ln \frac{m_{h 0}}{M_{Z}} \mathrm{GeV}$ was given (which also implied $\tan \beta \lesssim 1.3$ ).

It was pointed out by Barger et al. [12] that if indeed $m_{t}^{\text {pole }} \lesssim 160 \mathrm{GeV}$ (as suggested by the range given in Ref. [17] and here) then $m_{h^{0}}$ [in case (1)] is determined by the magnitude of the loop corrections, $\Delta_{h^{0}}$, and is $\lesssim 85 \mathrm{GeV}$. If so, the $b$-quark mass prediction in minimal $S U(5)$ (or similar) SGUT constrains the SM Higgs boson mass more significantly than the general MSSM (triviality) upper bound of $\sim 130 \mathrm{GeV}$ 18 20]. We agree qualitatively with this conclusion, but argue that two-loop ambiguities and important left-right $t$-scalar mixing effects, in addition to the higher $m_{t}^{\text {pole }}$ range, weaken the bound to $m_{h^{0}} \lesssim 110 \mathrm{GeV}$.

Below, we will establish the basis for and then pursue further numerical studies of the prediction for $m_{h^{0}}$ and its upper bound in the $\tan \beta \approx 1$ scenario. Rather than approximating the one-loop correction we will calculate $m_{h^{0}}$ for a given point in the parameter space using the supersymmetric spectrum (calculated numerically), and use monte-carlo routines to study the upper bound. In section 1 w we briefly review the MSSM Higgs potential and its

\footnotetext{
${ }^{4}$ That is unless one assumes a conspiracy of various corrections that are otherwise independent.
} 
minimization conditions. We specialize the discussion to the minimal SGUT scenarios, cases (1) and (2), and emphasize that the Higgs potential exhibits approximate global symmetries in these cases, on which we elaborate in Appendix A. We concentrate thereafter on the $\tan \beta \approx 1$ scenario. Some general features are described in section III. In particular, the large Higgsino mass parameter can generate false vacuum solutions with broken color and charge. We discuss that issue in greater detail in Appendix B. The calculation of the light Higgs boson mass in both the effective potential and renormalization group methods is discussed in section IV, where we use the comparison between the two methods to study the two-loop ambiguities in the calculation. The details of the two methods, as well as two-loop calculations, are further discussed in Appendix Q. Section $\nabla$ is reserved for further numerical studies of the Higgs boson mass, and we show that because of two-loop ambiguities and $\tilde{t}_{L}-\tilde{t}_{R}$ mixing $m_{h^{0}}$ can easily exceed $85 \mathrm{GeV}$ but, unless $m_{t}^{\text {pole }}$ is much heavier than the range suggested by (6), it remains in the range that may be relevant for LEPII. There is even some possibility that it is in the range still relevant for LEPI. We also discuss the relation between $t$-scalar mixing and color breaking. In particular, parameter ranges which correspond to large mixing (and large $m_{h^{0}}$ ) often have an unacceptable color-breaking global minimum, but not always. The discussion in section $\square$ is to a great extent independent of the other sections and can be read on its own. We will summarize our conclusions in section VI, where we also examine the implications for $m_{t}^{\text {pole }}$. In Appendix D we point out simple extensions of either the MSSM or the GUT in which our (constrained) analysis does not apply.

Below, we point out that (negative-energy) color-breaking local minima of the full scalar potential are generic. Approximate analytic constraints that are often used in the literature attempt to eliminate not only global but also local color-breaking minima, and are thus too strong. Furthermore, they are designed to eliminate only certain types of color-breaking minima, and are thus too weak. This is explicitly demonstrated in Appendix B, where we also compare analytic and numerical treatments of the problem.

We follow Ref. [8] in calculating the couplings (and the unification point), and Ref. [21] in treating the one-loop effective potential correction $\Delta V$, including contributions from all sectors. The boundary conditions at $M_{G}$ for $0 \leq m_{0} \leq 500 \mathrm{GeV},\left|A_{0}\right| \leq 3 m_{0}$, and $50 \leq M_{\frac{1}{2}} \leq 350 \mathrm{GeV}$, are picked at random. $m_{t}^{\text {pole }}$ values are picked at random but with a gaussian distribution defined by (6). For a given $m_{t}^{\text {pole }}, \tan \beta\left(M_{Z}\right)$ is picked at random within its allowed region [case (1)]. Values of $m_{t}^{\text {pole }}<155 \mathrm{GeV}$ are discarded so that $\tan \beta \gtrsim 1.1$ and the divergent limit is not reached (see below). We then solve iteratively for $\mu_{0}^{2}$ (and $B_{0}$ ). [The relevant renormalization group equations (RGE's) have been given by various authors [22].] The sign of $\mu_{0}$, which is a RG invariant, is picked at random as well. We explicitly verify that the potential is bounded from below; $S U(2) \times U(1)$ is properly broken; the lightest supersymmetric particle (LSP) is neutral (in practice, it is a neutralino); and that all the squared masses which correspond to physical scalars are positive before a point is accepted. We then find, in general, that we are in agreement with lower bounds coming from direct searches. (e.g., values of $M_{\frac{1}{2}} \lesssim 100 \mathrm{GeV}$, which would imply a too light chargino, are already excluded in our case by a tachionic $t$-scalar.) We do not impose a lower bound on $m_{h^{0}}$, which is the subject of our investigation. Our treatment of color and/or charge breaking (CCB) minima is discussed in Appendix B. 


\section{THE WEAK-SCALE HIGGS SECTOR}

The Higgs part of the MSSM (weak-scale) scalar potential reads 23, 18]

$$
\begin{gathered}
V\left(H_{1}, H_{2}\right)=\left(m_{H_{1}}^{2}+\mu^{2}\right)\left|H_{1}\right|^{2}+\left(m_{H_{2}}^{2}+\mu^{2}\right)\left|H_{2}\right|^{2} \\
+B \mu\left(H_{1} H_{2}+\text { h.c. }\right)+\frac{\lambda^{\mathrm{MSSM}}}{2}\left(\left|H_{2}\right|^{2}-\left|H_{1}\right|^{2}\right)^{2}+\Delta V,
\end{gathered}
$$

where $m_{H_{1}}^{2}, m_{H_{2}}^{2}$, and $B(\mu)$ are the soft (supersymmetric) mass parameters renormalized down to the weak scale, $m_{3}^{2} \equiv B \mu<0, \lambda^{\mathrm{MSSM}}=\frac{g_{2}^{2}+\frac{3}{5} g_{1}^{2}}{4}$, and we suppress $S U(2)$ indices. The one-loop correction 24, 18 $\Delta V=\Delta V^{\text {one-loop }}$ (which, in fact, is a threshold correction to the one-loop improved tree-level potential) can be absorbed to a good approximation in redefinitions of the tree-level parameters [21,25].

A broken $S U(2) \times U(1)$ (along with the constraint $m_{H_{1}}^{2}+m_{H_{2}}^{2}+2 \mu^{2} \geq 2\left|m_{3}^{2}\right|$ from vacuum stability) requires [23,18

$$
\left(m_{H_{1}}^{2}+\mu^{2}\right)\left(m_{H_{2}}^{2}+\mu^{2}\right) \leq\left|m_{3}^{2}\right|^{2}
$$

and the minimization conditions then give 23,18

$$
\begin{gathered}
\mu^{2}=\frac{m_{H_{1}}^{2}-m_{H_{2}}^{2} \tan ^{2} \beta}{\tan ^{2} \beta-1}-\frac{1}{2} M_{Z}^{2}, \\
m_{3}^{2}=-\frac{1}{2} \sin 2 \beta\left[m_{H_{1}}^{2}+m_{H_{2}}^{2}+2 \mu^{2}\right] .
\end{gathered}
$$

For $\tan \beta \rightarrow 1$ one has $h_{t} \gg h_{b}$, and hence cannot have $m_{H_{1}}^{2}=m_{H_{2}}^{2}$ (assuming universal initial conditions). Thus, $|\mu| \rightarrow \infty$ in that limit [case (1)], and the $S U(2) \times U(1)$ breaking is driven by the $B \mu$ term. In practice, we will stay away from the divergent limit in case (1) by taking $\tan \beta \gtrsim 1.1[21$. We do not expect significantly different results for $1 \leq \tan \beta \leq 1.1$; i.e., the divergence is either stabilized by model dependent finite-loop corrections or would exclude that region (see also below). For $\tan \beta \rightarrow \infty$ [case (2)] one has $B \mu \rightarrow 0$ so that the symmetry breaking is driven by $m_{H_{2}}^{2}<0$.

The $\tan \beta \rightarrow 1$ case corresponds to an approximate $S U(2)_{L+R}$ custodial symmetry of the vacuum [26,27, which we further discuss in Appendix A. The symmetry is broken at the loop level so that one expects $\tan \beta$ slightly above unity, in agreement with our cut $\tan \beta \gtrsim 1$.1. As a result of the symmetry, the CP-even Higgs mass matrix becomes

$$
M^{2} \approx \mu^{2} \times\left(\begin{array}{cc}
1 & -1 \\
-1 & 1
\end{array}\right)
$$

and it has a massless tree-level eigenvalue, $m_{h^{0}}^{T} \approx 0$. This is, of course, a well known result of the tree-level formula [23,18]

$$
m_{h^{0}}^{T}=\frac{1}{2}\left[m_{A^{0}}^{2}+M_{Z}^{2}-\sqrt{\left(m_{A^{0}}^{2}+M_{Z}^{2}\right)^{2}-4 m_{A^{0}}^{2} M_{Z}^{2} \cos ^{2} 2 \beta}\right]
$$


when taking $\beta=\frac{\pi}{4}$. The mass is then determined by the loop correction $m_{h^{0}} \approx \Delta_{h^{0}} \propto h_{t} m_{t}$ and is further studied in sections $\square$ and $\square$. On the other hand, in case (2) $\beta \rightarrow \frac{\pi}{2}$ and we have $m_{h^{0}}^{T} \approx M_{Z}$ (assuming $m_{A^{0}} \geq M_{Z}$ ). When adding the loop correction可 $m_{h^{0}} \lesssim \sqrt{2} M_{Z} \approx 130$ $\mathrm{GeV}$. The heavier CP-even Higgs boson mass eigenvalue equals approximately $\sqrt{2}|\mu|$ in case (1) and $m_{H^{0}} \approx m_{A^{0}}$ in case (2). The loop corrections are less relevant here as typically $m_{H^{0}}^{2} \gg \Delta_{H^{0}}^{2}$ (in particular, when $|\mu| \rightarrow \infty$ ).

The custodial symmetry (or the large $\mu$ parameter) dictates in case (1) a degeneracy $m_{A^{0}} \approx m_{H^{0}} \approx m_{H^{+}} \approx \sqrt{2}|\mu|$. [The tree-level corrections to that relation are of order $\left(M_{W, Z} / m_{A^{0}}\right)^{2}$.] That is, at a scale $Q \approx \sqrt{2}|\mu| \approx 2 \mathrm{TeV}$ the heavy Higgs doublet $H$ is decoupled, and the effective field theory below that scale has only one massless $\left(m_{h^{0}}^{T} \approx 0\right)$ Coleman-Weinberg SM-like $\left(\nu_{h^{0}}=\nu\right)$ Higgs doublet, $h$. Unlike in our case, in general $M_{Z} / m_{A^{0}}$ is not guaranteed to be small, and the decoupling scale $Q$ is much lower, typically below the scalar-quark thresholds.

The Higgs sector in case (2) exhibits an approximate $O_{4} \times O_{4}$ symmetry (see Appendix A]. This is a special case of the $m_{3}^{2} \rightarrow 0$ case discussed in Ref. [28]. To obtain $m_{3}^{2} \approx 0$ (starting with universal initial conditions and $h_{b} \approx h_{t}$ ) one would require large $M_{\frac{1}{2}}$ and $\left|\mu_{0}\right|$, which enable one to obtain the desired limit by adjusting large cancellations [29 31]. Once expectation values are acquired, $A^{0}$ and $H^{+}$are massive pseudo-Goldstone bosons of the broken $O_{4}$ symmetry. However, they are typically heavy (for $\nu_{\text {down }}=0$ the $O_{4}$ symmetry is restored) but lighter than the scalar quarks in this scenario (whose mass $m_{\tilde{q}} \approx$ $\left.\sqrt{m_{0}^{2}+6 M_{\frac{1}{2}}^{2}} \approx 1 \mathrm{TeV}\right)$.

Large $\tan \beta$ [case (2)] solutions are discussed in Ref. [29,30, 13, 32, 31]. They predict $m_{t}^{\text {pole }} \approx 180 \pm 15 \mathrm{GeV}$ [8,13], heavy scalars, and contain potentially troublesome loop corrections, e.g., to $m_{b}, b \rightarrow s \gamma$ [13,32]; and, in the absence of light scalars to counterbalance large $m_{t}$ contributions, also to $Z \rightarrow b \bar{b}$ (see Ref. [33]). The former can eliminate the motivation for any Yukawa coupling analysis. A different approach was recently presented in Ref. [31] where those large finite-loop corrections were used to construct models consistent with universality, a broken $S U(2) \times U(1)$, and $h_{t}=h_{b}=h_{\tau}$. Finite superpartner $(\sim 1 \mathrm{TeV})$ loops are adjusted to diminish $m_{b}$ by the right amount $(\sim 1 \mathrm{GeV})$, and $m_{b}$ is strongly dependent on the values of the soft parameters, a possible but undesirable situation. Much lower values of $m_{t}^{\text {pole }}$ are then preferred. Diminishing $m_{t}$ may rectify the situation with $Z \rightarrow b \bar{b}$, but the prediction for the $b \rightarrow s \gamma$ branching ratio can be significantly modified by the same superpartner loops [13]. In contrast, the $\tan \beta \approx 1$ [case (1)] solution constrains only $m_{t}^{\text {pole }} \gtrsim 140$ $\mathrm{GeV}\left(m_{t}^{\text {pole }} \gtrsim 155 \mathrm{GeV}\right.$ when imposing the $\left.\tan \beta \gtrsim 1.1 \mathrm{cut}\right)$, is in better agreement with (6), and predicts a light Higgs boson $\left(m_{h^{0}} \lesssim 110 \mathrm{GeV}\right)$. Cases (1) and (2) are summarized and compared in Table $\llbracket$. We hereafter study the $\tan \beta \approx 1$ [case (1)] solution only. We assume that finite-loop corrections to $m_{b}$ are at the most a few percent which is, in general, the case away from the large $\tan \beta$ limit, and thus do not alter our results. [The parameter space is sensitive to those corrections only in case (2).]

\footnotetext{
${ }^{5} \Delta_{h^{0}}$ is enhanced in this case by the large $m_{t}$ and the heavy $t$-scalars.
} 


\section{THE $\tan \beta \rightarrow 1$ SCENARIO}

Our primary motivation to study the $\tan \beta \approx 1$ solution to the MSSM is its consistency with condition (2). The large values of $\alpha_{s}\left(M_{Z}\right)$ implied by (11) [e.g., Eq. (4)] generate (too) large positive loop corrections to $h_{b} / h_{\tau}$, which need to be counterbalanced by large negative corrections $\propto h_{t}^{2}, h_{b}^{2}$. The latter are sufficient only if some of the the Yukawa couplings are large, i.e., near their unitarity upper bounds, which is the case in the allowed regions. [Also, $H_{\alpha_{s}}$ in (4) becomes non-negligible in that limit.] The large values of the Yukawa couplings can be understood in terms of a quasi-fixed point in the flow of the respective RGE's [34]. In particular, in case (1) $h_{t}$ is near its quasi-fixed point. The two issues, i.e., the consistency of $\tan \beta \approx 1$ with (2) and the $h_{t}$-RGE flow structure, are strongly related.

The smallness of $\Delta_{\beta}$ (or alternatively, the large $|\mu|$ ) can be understood in terms of an approximate $S U(2)_{L} \times S U(2)_{R}$ symmetry in the Higgs potential, which, if it exists at some scale, will be only slightly broken at $M_{Z}$, as is shown in Appendix $\mathrm{A}$. The $\tan \beta \approx 1$ scenario was previously studied in Ref. [35], where it was referred to as "highest classical degeneracy": in the $\tan \beta=1$ limit condition (8) becomes an equality. [In practice, we find that $\left(m_{H_{1}}^{2}+\mu^{2}\right)\left(m_{H_{2}}^{2}+\mu^{2}\right) / m_{3}^{4} \gtrsim 0.99$ for $\Delta_{\beta}<1$ and after the proper redefinitions.] It was subsequently studied in Ref. 36] in the context of a global MSSM (i.e., no high-scale assumptions), and its consistency with limits on $m_{h^{0}}$ was shown. The $\tan \beta \approx 1$ solution suggested by the minimal SGUT relation $h_{b}=h_{\tau}$ revived the interest in that corner of parameter space. More recently Ref. [37 39], motivated by the quasi-fixed point prediction to $m_{t}$, extended the discussion from the Higgs sector to the full parameter space.

In Figs. 2a and b we show the prediction for $\mu$ as a function of $m_{t}^{\text {pole }}$ and of $\tan \beta$, respectively. Typically $|\mu| \sim 1 \mathrm{TeV}$, depending on $\Delta_{\beta}$ and on the soft parameter scale. It is the large $|\mu|$ parameter that dictates the characteristics of the scenario (and not only for the Higgs sector). Before further elaborating on the calculation of $m_{h^{0}}$, let us briefly summarize some of the features that appear in our numerical studies, and which are of relevance for the discussion below. Though we agree on the characteristics with other authors, our emphasis and interpretation are different.

1. The Higgsino (with mass $\sim \mu$ ) is decoupled from the (much lighter) gauginos. The bino and the wino are the lightest neutralino and chargino, respectively. (The former is the LSP which has a bino fraction near unity.) The heavy Higgsino decouples from the $Z \rightarrow b \bar{b}$ vertex, and thus, smaller values of $m_{t}^{\text {pole }}$ are favored . However, $m_{t}^{\text {pole }}$ and $\tan \beta$ are correlated, and as $m_{t}^{\text {pole }}$ grows $|\mu|$ is diminished (see Fig. 2). Thus, in principle, Higgsino $-t$-scalar loops can still counterbalance large $m_{t}$ contributions to $Z \rightarrow b \bar{b}$ (see, for example, Ref. [33]) if the $t$-scalar is light enough. [A light $t$-scalar is, however, less likely if $|\mu|$ is small (see below) and from the $\rho$-parameter.]

2. The $t$-scalar mass-squared matrix is (we take all parameters to be real)

\footnotetext{
${ }^{6}$ The prediction for the $Z \rightarrow b \bar{b}$ branching ratio decreases with $m_{t}^{\text {pole }}$, and its measured value is $\sim 1.5$ s.d. higher than the SM prediction with $m_{t}^{\text {pole }} \approx 140 \mathrm{GeV}$. The discrepancy grows with $m_{t}^{\text {pole }}$ 40.
} 


$$
\left(\begin{array}{cc}
m_{\tilde{t}_{L}}^{2} & m_{t}\left(A_{t}+\mu / \tan \beta\right) \\
m_{t}\left(A_{t}+\mu / \tan \beta\right) & m_{\tilde{t}_{R}}^{2}
\end{array}\right),
$$

and similarly for the superpartners of the other fermions. The diagonal elements correspond to the left and right-handed $t$-scalar squared masses, $m_{\tilde{t}_{L, R}}^{2}$, which consist of soft, $F$, and $D$ terms. $A_{t}$ is the trilinear soft parameter preceeding the $h_{t} H_{2} \tilde{t}_{L} \tilde{t}_{R}$ term in the scalar potential, with $A_{t}\left(M_{G}\right)=A_{0}$. The prediction for the diagonal terms has no unique charachteristics in our case, and the left and right-handed masses are in the $\sim 100 \mathrm{GeV}-1 \mathrm{TeV}$ range. However, the mixing term is typically large (unless the fermion mass is $\approx 0$ as is the case for the light families). In the limit $|\mu| \rightarrow \infty$ there is a $\theta_{t}=\frac{\pi}{4}$ left-right mixing so that one of the eigenstates would have a negative mass squared, and the limit cannot be fully realized. In practice, the soft terms and $m_{t}|\mu|$ are often of the same order of magnitude, with a nearly degenerate mass-squared matrix. Thus, the requirement that (9) defines a physical minimum with no negative squared masses, corresponding to physical scalars, constrains $\mu$. Also, our previous comment regarding a light $t$-scalar and a small $\mu$-parameter is clear by observation of (12). It is interesting to note that by fine adjustments of the soft parameters [i.e., of the degeneracy in (12)] one may realize a scenario with a light $(\lesssim 45 \mathrm{GeV}) t$-scalar which is nearly decoupled from the $Z$ (e.g., $\sin \theta_{t} \approx 0.8$ ). (See, for example, Ref. [41].) However, such scenarios are often associated with false vacua (see below and Appendix B).

3. The absence of a negative $t$-scalar squared mass determines a $\left(m_{0}\right.$ and $\Delta_{\beta}$ dependent) lower bound on $M_{\frac{1}{2}}$. In particular, "no-scale" (i.e., $m_{0}=A_{0}=0$ ) SGUT models are strongly constrained $[$. Also, requiring that the light eigenstate of the $\tau$-scalar masssquared matrix (which carries charge) is not the LSP determines an upper bound on $M_{\frac{1}{2}}$ in the no-scale limit (see also Kane et al. [20]). These point at a correlation between the bounds on the soft parameters. A different class of correlations which take effect in the $h_{t} \rightarrow h_{t}^{\text {fixed }}$ limit is discussed by Carena et al. [37]. Strong correlations (e.g., the $m_{t}^{\text {pole }}-\tan \beta$ correlation? , the $h_{t} \rightarrow h_{t}^{\text {fixed }}$ ones, and those due to scalar mixings discussed here) do not allow elimination of the large- $|\mu|$ solutions (that correspond to large left-right mixing enhancement to $m_{h^{0}}$ - see below) on the basis of fine-tuning arguments. The naive notion of fine-tuning becomes obscure and misleading in the presence of strong correlations. (See also Ref. [37].) Rather, strong correlations make the scenario quite predictive.

4. We can rewrite the mixing term in (12) as $h_{t} H_{2}^{0} \hat{\Gamma}$ where $H_{2}^{0}=\nu_{u p} . \hat{\Gamma}$ is strongly constrained by requiring that the full scalar potential does not have a color and/or

7 The strict no-scale assumption $B_{0}=m_{0}=A_{0}=0$ is not consistent here. See Appendix A.

${ }^{8}$ No-scale models with $h_{b}=h_{\tau}$ were recently discussed in Ref. 42

${ }^{9}$ Note that even if we do not require $h_{b}=h_{\tau}, m_{t}^{\text {pole }}$ and the lower bound on $\tan \beta$ are correlated due to unitarity considerations. 
charge breaking global (GCCB) minimum, which is a different issue than whether the extremum defined by (9) is indeed a minimum. The issue of false vacua is of particular interest in the $\tan \beta \rightarrow 1$ scenario as $V\left(H_{1}, H_{2}\right) \rightarrow 0^{-}$in that limit. Below, we will consider that subject in some detail. In particular, see Appendix $\mathbb{B}$. We find that the absence of negative energy CCB minima implies the constraint, first described in Ref. [43],

$$
\left(\left|A_{t}\right|+s|\mu|\right)^{2} \leq 2\left(m_{\tilde{t}_{L}}^{2}+m_{\tilde{t}_{R}}^{2}\right)
$$

where $s=A_{t} \mu /\left|A_{t} \mu\right|,\left(\left|A_{t}\right|+s|\mu|\right)^{2}=\hat{\Gamma}^{2}$ (for $\tan \beta=1$ ), and $m_{\tilde{t}_{L, R}}^{2}$ consist of only the soft terms. (Note the importance of the trilinear parameter and of the relative sign.) Our numerical studies imply that (13) is (to a good approximation) a sufficient condition (in that region of parameter space) but is not necessary (i.e., it is too restrictive). For example, more than $80 \%$ of the points that are inconsistent with that constraint for $m_{t}^{\text {pole }} \gtrsim 165 \mathrm{GeV}$ correspond to CCB minima which are only local and are therefore safe. In Fig. 2 only filled squares correspond to (probably unacceptable) GCBB minima. Also observe in Fig. 2 that $\mu<0$ is preferred by CCB constraints. This is because typically $A_{t} \gg A_{0}$ for $A_{0}<0$ so that the contribution from a large and positive $\mu$ cannot be canceled in (13) (for our range of $A_{0}$ ).

\section{THE LOOP-INDUCED MASS}

Let us now proceed to discuss the mass of the SM-like higgs boson. The upper bound on $m_{h^{0}}$ is given, e.g., in Ref. [44,

$$
m_{h^{0}}^{2} \leq M_{Z}^{2} \cos ^{2} 2 \beta+\frac{3 \alpha m_{t}^{4}}{4 \pi s^{2}\left(1-s^{2}\right) M_{Z}^{2}}\left\{\ln \left(\frac{m_{\tilde{t}_{1}}^{2} m_{\tilde{t}_{2}}^{2}}{m_{t}^{4}}\right)+\Delta_{\theta_{t}}\right\}
$$

where

$$
\begin{gathered}
\Delta_{\theta_{t}}=\left(m_{\tilde{t}_{1}}^{2}-m_{\tilde{t}_{2}}^{2}\right) \frac{\sin ^{2} 2 \theta_{t}}{2 m_{t}^{2}} \ln \left(\frac{m_{\tilde{t}_{1}}^{2}}{m_{\tilde{t}_{2}}^{2}}\right) \\
+\left(m_{\tilde{t}_{1}}^{2}-m_{\tilde{t}_{2}}^{2}\right)^{2}\left(\frac{\sin ^{2} 2 \theta_{t}}{4 m_{t}^{2}}\right)^{2}\left[2-\frac{m_{\tilde{t}_{1}}^{2}+m_{\tilde{t}_{2}}^{2}}{m_{\tilde{t}_{1}}^{2}-m_{\tilde{t}_{2}}^{2}} \ln \left(\frac{m_{\tilde{t}_{1}}^{2}}{m_{\tilde{t}_{2}}^{2}}\right)\right],
\end{gathered}
$$

and where $m_{\tilde{t}_{i}}^{2}$ are the eigenvalues of the $t$-scalar mass-squared matrix, $\theta_{t}$ is the mixing angle, and we have neglected other loop contributions. (They are included in our numerical calculations below.) The tree-level mass squared, $m_{h^{0}}^{T}{ }^{2}$, and the loop correction, $\Delta_{h^{0}}^{2}$, are bounded by the first and second terms on the r.h.s. of Eq. (14), respectively. In the absence of mixings $\Delta_{\theta_{t}}=0$. For $\tan \beta \rightarrow 1$ one obtains $m_{h^{0}}^{T} \rightarrow 0$, and thus $m_{h^{0}} \approx \Delta_{h^{0}}$.

The upper bound on $\Delta_{h^{0}}$ scales as $m_{t}^{4}$ and depends on the overall scale of the $t$-scalar mass, the amount of left-right mixing, and the separation $\left|m_{\tilde{t}_{1}}^{2}-m_{\tilde{t}_{2}}^{2}\right|$. The large mixing and separation for $|\mu| \rightarrow \infty$ enhance $\Delta_{h^{0}}$ significantly (typically by $10-20 \mathrm{GeV}$ ) compared to the first term alone (which was all that was included in Ref. [12]). Condition (2) (and $h_{t}$ unitarity) correlate $m_{t}^{\text {pole }}$ and $\tan \beta$ in case (1) (see Ref. [8,16]), and $\Delta_{\beta}$ increases from $\sim 0$ for $m_{t}^{\text {pole }} \sim 140 \mathrm{GeV}$ to $\sim 1$ for $m_{t}^{\text {pole }} \sim 190 \mathrm{GeV}$. By increasing $\tan \beta$ one gradually departs 
from the $|\mu| \rightarrow \infty$ limit, and the enhancement to $\Delta_{h^{0}}$ from left-right mixing decreases. Thus, there is an interplay between $\Delta_{\theta_{t}}$ and the overall factor of $m_{t}^{4}$. (Of course, $m_{h^{0}}^{T}$ grows with $\tan \beta$ and hence with $m_{t}^{\text {pole }}$.)

As was pointed out in the previous section (see also Appendix B), $\mu$ and the amount of mixing $\hat{\Gamma}$ are subject to color and/or charge breaking (CCB) constraints. In this section points which are consistent with (13), i.e., which have no negative-energy CCB minima, are marked by a circle $-O$. Points which are inconsistent with (13) and therefore have either a local or global negative-energy CCB minimum are marked by a "diamond" $-\diamond$. The filled diamonds correspond to a global color breaking minimum (as determined using numerical procedures), which is (most likely) unacceptable. The open diamonds represent CCB minima which are only local (i.e., above the standard model minimum) and are therefore safe. Constraint (13) is always evaluated at the $t$-scalar scale, independent of the scale chosen for minimization. The same is true for the numerical search of global CCB (GCCB) minima.

Calculating $m_{h^{0}}$ is reduced (in our case) to a great extent to calculating $\Delta_{h^{0}}$, i.e., the deviation from what would be the case if the matter were supersymmetric at the weak scale [e.g., $m_{\tilde{t}_{1}}=m_{\tilde{t}_{2}}=m_{t}$ in (14) and (15)]. Two standard ways to perform the calculation are the effective potential method (EPM) (e.g., see Ref. [45, 44, 46, 36]) and the renormalization group method (RGM) (e.g., see Ref. 47,48,36]). The two methods correspond to a "run and diagonalize" and "diagonalize and run" algorithm, respectively. The algorithms and their implementations are discussed and compared in greater detail in Appendix Q . Here we would like to demonstrate that the EP and RG methods do agree (within their domains of validity), i.e., the results are not sensitive to the way one treats the threshold corrections. In particular, both methods exhibit a large $(10-20 \%)$ two-loop ambiguity from the presence of some twoloop residual terms in the calculations (next-to-leading and leading two-loop logarithms, as well as finite terms). We explicitly identify and discuss such terms in Appendix C. The residual logarithmic two-loop terms can be described in terms of a scale ambiguity (i.e., the scale dependence of the one-loop calculation is of two-loop order). Indeed, comparing the two methods and studying that ambiguity allow us to estimate the two-loop correction. However, the uncertainty remains.

In the EPM the ambiguity is explicitly related to a scale dependence, as one is required to specify a subtraction scale $Q$ (i.e., halt running at $Q$ and diagonalize mass matrices). Indeed, including $\Delta V^{\text {one-loop }}(Q)$ corrects for one-loop leading logarithms, and thus guarantees a scale independence of the calculation, but only to that order (and up to wave-function renormalization). It is suggestive to take $Q=M_{Z}$, i.e., the scale at which the physical inputs are given. Another attractive choice is a scale at which $\Delta V^{\text {two-loop }}(Q)$ is minimized. We will not attempt to find such a scale rigorously, but estimate $Q \approx 600 \mathrm{GeV}$. (One indeed expects such a $Q$ to be in the vicinity of some average scalar-quark scale, as for $\Delta V^{\text {one-loop }}$ in Ref. [49].) We will use the comparison between the two methods to justify our choice. (Of course, there is no one choice that is suitable for all sets of parameters.)

In the RGM, which sums the leading logarithms to all orders if one integrates numerically, the ambiguity is more apparent in terms of orders in perturbation theory (i.e., the order at which the summation is truncated in approximate iterative solutions). However, there is also an explicit scale dependence from the choice of decoupling scales for the heavy fields (i.e., decouple heavy eigenstates, calculate RGE's, and run to the next decoupling scale). We will decouple the heavy Higgs doublet $H$ at $m_{A^{0}} \approx 2.1 \mathrm{TeV}$ and other fields at lower 
thresholds $\square$. When decoupling $H$ we include appropriate matching conditions [48].

The EPM is more straightforward to implement (and is therefore more common in the literature) while the RGM requires a careful consideration of decoupling scales and matching conditions.

The scatter plot Fig. 3 displays the results of an EPM calculation with $Q=M_{Z}$. (When increasing $Q$ the soft mass parameters and $m_{t}$ decrease so that the EPM $m_{h^{0}}$ is diminished.) The scatter plot Fig. 1 displays the results of a RGM calculation (performed numerically). Comparing them, one observes a $10-20 \mathrm{GeV}$ difference between the respective Higgs boson masses (for a given point in the parameter space). However, this is not due to the different choices of an algorithm, but to the different residual two-loop dependences in the two calculations. To illustrate the maximum ambiguity we used the $t$-quark pole (running) mass in the calculations presented in Fig. 3 (Fig. 4). (The $\sim 5 \%$ difference in the $t$-quark masses is formally of two-loop order.) In both plots one can observe a separation into a heavier and a lighter Higgs boson branches. This is due to the enhancement to $m_{h^{0}}$ in some cases from $\tilde{t}_{L}-\tilde{t}_{R}$ mixing, and is further discussed in the following section.

To further compare the two methods, it is useful to consider the following exercise. The RGM calculation could be modified so that leading logarithms are not summed to all orders, e.g., one could use the one-loop leading-logarithm approximation formula of Ref. [47. Alternatively, we could minimize the unsummed $\ln \left(m_{\tilde{t}} / Q\right)$ leading (and/or next-toleading) logarithms in the EPM by choosing $Q \approx m_{\tilde{t}}$. In either case the residual terms in the different calculations are qualitatively brought on the same footing. We carried out that exercise numerically, and Fig. 5 (Fig. 6) compare the EPM with $Q=M_{Z}(Q=600 \mathrm{GeV})$ calculation to the RGM leading logarithm approximation (numerical calculation). Once the residual two-loop dependence of the calculation is properly adjusted, the two algorithms agree well, in particular for $Q \approx 600 \mathrm{GeV}$. [All calculations presented in Fig. 5 (Fig. 6) use the $t$-quark pole (running) mass.]

This all points at the most important source of uncertainty: residual two-loop (and wave-function) scale dependences and a $\sim 5 \% \alpha_{s}$ dependence of $m_{t}$. Two-loop terms fuel the ambiguity, which therefore cannot be removed in a satisfactory fashion within a one-loop calculation. (Even though we could identify some two-loop terms, the consistency of their removal is doubtful). Expanding the calculation to higher orders is not straightforward. We discuss two-loop calculations in Appendix O.

Figs. 3 and 14 define the theoretical ambiguity in determining the loop induced $m_{h^{0}}$ in this scenario. While the former sets the upper bound (for a given $m_{t}^{\text {pole }}$ ), the latter is a more accurate leading-logarithm calculation (and uses properly the running $t$-quark mass). However, within a one-loop calculation, and in particular when next-to-leading logarithms and some finite terms are comparable to higher-loop leading logarithms (which is the case here - see Appendix ( ), neither method provides a more consistent calculation then the other. As we argue in Appendix Q, the former is also an upper bound on twoloop calculations, which most probably diminish $m_{h^{0}}$. We do not think that any stronger statements are justified at present. We continue to discuss the prediction and its upper

\footnotetext{
${ }^{10}$ We checked that the details of the choices for those intermediate scales introduce a $\sim 1 \%$ uncertainty.
} 
bound in the next section.

A different issue is that of the constraints on the $t$-scalar left-right mixing enhancement to $\Delta_{h^{0}}$. In the EPM [e.g., Eq. (14)] the enhancement is a straightforward result of diagonalizing the CP-even mass matrix. In the RGM one has to define the decoupling scale for the $t$ scalars. If $m_{\tilde{t}_{1}} \gg m_{\tilde{t}_{2}}$ then one would decouple only $\tilde{t}_{1}$ while $\tilde{t}_{2}$ loops could still contribute to $\Delta_{h^{0}}$. (In practice, the effect is accounted for in the RGM by a set of appropriate matching conditions.) However, the amount of mixing $\hat{\Gamma}$ (and thus the enhancement) is strongly constrained by the physical vacuum.

Requiring that the SM extremum is a minimum (i.e., no negative squared masses corresponding to physical degrees of freedom), and furthermore, is the global minimum (i.e., there is no deeper minimum that does not conserve color and/or charge) strongly constrains $\hat{\Gamma}$. Constraints imposed by the latter may be evaded $\amalg$ if the non-SM global minimum is separated from the SM local minimum by a tunneling time greater than the age of the universe [50]. Constraint (13) (naively) attempts to eliminate all, i.e., local and global, negative-energy CCB minima in that region of parameter space and is too strong. The points marked in the scatter plots by only a an open "diamond" survive a more careful analysis, i.e., they correspond to only local minima [12. We find, using numerical methods, that for $m_{t}^{\text {pole }} \lesssim 165 \mathrm{GeV}\left(165 \mathrm{GeV} \lesssim m_{t}^{\text {pole }}\right)$ roughly $80 \%$ (15\%) of the points that fail to satisfy constraint (13) correspond to (probably unacceptable) GCCB minima of the (oneloop improved) tree-level scalar potential. The enhancement is then partially washed away by requiring consistency with the physical vacuum. The possibilty of coexistence of the two vacua (with a tunneling time greater than the age of the universe) is, at the present stage of our calculation, a remaining ambiguity. We further discuss those issues in the next section and, in greater detail, in Appendix B.

\section{THE PREDICTION FOR THE HIGGS BOSON MASS AND ITS UPPER BOUND}

In this section we present and discuss our numerical results. In section [V] we discussed various methods to calculate the mass (to one-loop). The effective potential method (EPM) with a subtraction scale $Q=M_{Z}$ (e.g., see Fig. 3) puts the weaker constraints on $m_{h^{0}}$ and is the conservative choice for the upper bound. In Fig. 7 we present monte-carlo distributions of $m_{h^{0}}$ using that method for $155 \leq m_{t}^{\text {pole }} \leq 165,165 \leq m_{t}^{\text {pole }} \leq 175$, and $175 \leq m_{t}^{\text {pole }} \leq 185$ $\mathrm{GeV}$. The upper bound can be read off the figures, i.e., $m_{h^{0}} \lesssim 101,106,114 \mathrm{GeV}$ for $m_{t}^{\text {pole }} \lesssim$ $165,175,185 \mathrm{GeV}$, respectively. (Note that these bounds are deduced from our monte-carlo calculations, and are not rigorous.) Nevertheless, we would like to stress that typically $m_{h^{0}}$ lies below its upper bound. Indeed, one cannot rule out the possibility (if $m_{t}^{\text {pole }}$ is heavy

\footnotetext{
${ }^{11}$ This claim, however, does not take into account finite-temperature effects.

12 In fact, all points are found to have at least a negative-energy CCB local minimum of type $M^{2}=0$ - see Appendix B. However, we find that in our region of the parameter space (13) is a useful tool to identify minima that compete with the standard model minimum.
} 
enough) that $m_{h^{0}}$ is just outside the range that LEPII may cover. However, this is not likely on both grounds, the $m_{t}^{\text {pole }}$ distribution [Eq. (6)] and the $m_{h^{0}}$ (theoretical) distribution (as reflected in Fig. (7).

The role of the large left-right mixing in the $t$-scalar sector can be seen in Figs. Ia and 7b, which exhibit two peaks in the mass distribution, a "logarithmic" and a "mixing" peak. The former is due to the diagonal entries in the $t$-scalar mass matrix [Eq. (12)] while the latter, which is at larger mass, is due to the mixing enhancement $\left[\Delta_{\theta_{t}}\right.$ in Eq. (15)]. The interplay between the mixing and the $m_{t}^{4}$ factor in the loop correction $\Delta_{h^{0}}$ [e.g., in Eq. (14)], and between $\Delta_{h^{0}}$ and the tree-level mass $m_{h^{0}}^{T}$ (that grows with $\tan \beta$ and, therefore, with $m_{t}^{\text {pole }}$ ) can be seen by comparing Figs. Za-b with Fig. Zc. The mixing enhancement plays an important role for smaller values of $m_{t}^{\text {pole }}(\Delta \beta \rightarrow 0)$. In fact, that interplay generates for a fixed set of soft parameters a local minimum in the $m_{h^{0}}$ distribution (not shown in the figures) near $m_{t}^{\text {pole }} \approx 165 \mathrm{GeV}$.

The left-right mixing enhancement is strongly constrained when requiring that the minimum of the full scalar potential is the physical one, i.e., no fields other than $H_{1}^{0}=\nu_{\text {down }}$ and $H_{2}^{0}=\nu_{u p}$ have non-vanishing expectation values (see section $\mathbb{I I}$ and Appendix B). Constraint (13) eliminates potentially dangerous points in the parameter space (marked by a "diamond" in Figs. (3 and (1), some of which, however, correspond to only a local colorbreaking minimum (marked by an open "diamond"). Those which correspond to global minima are marked by a filled "diamond". Fig. 8 is the same as Fig. 7 except that points which correspond to global color and/or charge breaking (GCCB) minima are omitted (the shaded areas have no negative-energy CCB minimum, global or local.) The mixing peak has diminished significantly but has not disappeared. Its presence is even stronger for larger values of $m_{t}^{\text {pole }}$ (see appendix $\mathbb{B}$ ). (Some caution is required: the cosmological history of the universe may accommodate such a global minimum.)

A different issue is that of the large two-loop ambiguity in the calculation pointed out in section $\mathbb{E V}$. For example, in Fig. 9 we show the same distributions as in Fig. (7, but in which the running (rather than the pole) $t$-quark mass was used to calculate $m_{h^{0}}$. (The running mass is $\sim 5 \%$ smaller.) Also, the distribution corresponding to the renormalization

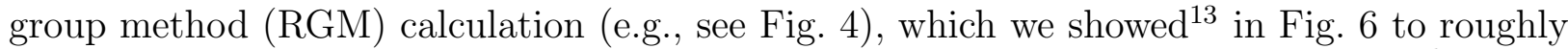
agree with the EPM calculation (with $Q \approx m_{\tilde{t}}$ ), is shown in Fig. 10 for $155 \leq m_{t}^{\text {pole }} \leq 165$ and $165 \leq m_{t}^{\text {pole }} \leq 175$, respectively. Again, the $t$-quark running mass is used. Also shown are the distributions when GCCB points are omitted (shaded areas) to be compared with the total areas (shaded and unshaded) in Fig. 8. Note the concentration at lighter masses compared to previous figures, even though the upper limits are only slightly changed. It should be stressed that had we not included the proper RGM matching functions [48] (which would roughly correspond to setting the left-right mixings to zero by hand) then the RGM distribution would sharply peak at the lower end of the $m_{h^{0}}$ range. Thus, the importance of the matching functions is obvious.

All, Figs. 7- 10, are consistent within the theoretical ambiguity. It is suggestive that a

13 The RGM calculation sums the leading logarithms to all orders in perturbation theory, while in the EPM calculation with $Q \approx m_{\tilde{t}}$ higher order terms are, in principle, minimized, and do not need to be summed. 
consistent two-loop calculation will reduce the upper bounds given by the EPM calculation (we choose conservatively Fig. 7), but probably not to lower values than suggested by the RGM (Fig. 10). Further study is required before any stronger conclusions can be drawn. Even so, for $m_{t}^{\text {pole }} \lesssim 175 \mathrm{GeV} m_{h^{0}}$ [in case (1)] is within the reach of LEPII, and possibly of the Tevatron (see Ref. [51]). If $m_{t}^{\text {pole }}$ is lighter, as suggested by electroweak precision data, our histograms imply that $m_{h^{0}}$ may still be relevant for Higgs searches at LEPI. (In particular, if two-loop corrections reduce $m_{h^{0}}$ as suggested by Fig. 10.)

\section{CONCLUSIONS}

To summarize, we worked in a constrained framework assuming (up to high and low-scale threshold corrections) $\alpha_{1}=\alpha_{2}=\alpha_{3}=\alpha_{G}, h_{b}=h_{\tau}$, and universal initial conditions. Also, we explicitly included the correlation between the $t$-quark mass and the weak angle from electroweak precision data. Within that framework, only $\tan \beta \approx 1$ and large $\tan \beta$ regions are allowed. In the large $\tan \beta$ region (which is allowed only for a large $m_{t}^{\text {pole }}$, unless $m_{b}$ is allowed to get large finite corrections from superpartner loops) $m_{h^{0}}$ is bounded by the triviality bound, and is probably not interesting for Higgs searches at LEPII.

In the $\tan \beta \approx 1$ region the tree-level $m_{h^{0}}^{T}$ nearly vanishes, but grows with $m_{t}^{\text {pole }}$. The loop correction to the mass is enhanced by large $\tilde{t}_{L}-\tilde{t}_{R}$ mixing terms. The latter are diminished with an increasing $m_{t}^{\text {pole }}$ but, as $m_{t}^{\text {pole }}$ grows, the overall factor of $m_{t}^{4}$ can compensate for that effect. The enhancement due to the large mixing is further constrained by requiring the physical vacuum to correspond to the global minimum of the full scalar potential. We find that for parameters corresponding to large mixing there is usually a color breaking minimum, but in many cases this is only a (presumably harmless) local minimum. We also pointed out that a two-loop ambiguity in the calculation of the Higgs boson mass is enhanced in our case and can be as large as $20 \mathrm{GeV}$. All these effects lead to the conservative bound $m_{h^{0}} \lesssim 100$ (110) GeV for $m_{t}^{\text {pole }} \lesssim 160$ (175) GeV. (We briefly discuss two-loop calculations in Appendix 9 , where we conclude that such a calculation will most probably strengthen the upper bound.) The Higgs boson mass in the class of models characterized by $\tan \beta \approx 1$ is then almost certainly within the reach of LEPII (and possibly within the reach of the Tevatron [51]). Furthermore, the Higgs boson is typically lighter than the upper bound, and may be light enough to still be discovered at LEPI.

Regarding the $t$-quark mass, one requires for $S U(2) \times U(1)$ breaking (in this scenario) $m_{t}^{\text {pole }} \gtrsim 140 \mathrm{GeV}$, and further requiring $\tan \beta \gtrsim 1.1$ (which is the case if the divergent limit is to be avoided, i.e., if the divergence is not stabilized by model-dependent finite terms) we obtain $m_{t}^{\text {pole }} \gtrsim 155 \mathrm{GeV}$. Eq. (6) and, in particular, the structure of the radiative corrections to the $Z \rightarrow b \bar{b}$ vertex, however, suggest that $m_{t}^{\text {pole }}$ is not much larger. (The latter can be evaded only if both the $t$-scalar and the Higgsino are light, which is not very likely in our case.) Also, we were able to understand the smallness of the parameter space allowed by $h_{b}=h_{\tau}$ in terms of a custodial symmetry. The symmetry (theoretical) argument suggests

$m_{t}^{\text {pole }} \lesssim 185 \mathrm{GeV}\left(\Delta_{\beta} \lesssim 1\right)$. In Appendix $\mathrm{D}$ we briefly examine extended Higgs sectors, and point out that the Higgs mass is a possible probe of such extensions if $m_{t}^{\text {pole }}$ (and/or $\tan \beta$ ) is known.

In Appendix B we point out that the full (one-loop improved) scalar potential generically possesses color and/or charge breaking negative-energy local minima. Analytic constraints 
that are often found in the literature attempt to eliminate all such local minima (i.e., are too strong) but by construction apply to only certain types of minima while ignoring others (i.e., are too weak). We give a general classification of the local minima and are able to further identify [in case (1)] those that can potentially compete with the standard model minimum.

\section{ACKNOWLEDGMENTS}

This work was supported by the US Department of Energy Grant No. DE-AC02-76ERO-3071. It is pleasure to thank J. Erler for useful discussions concerning electroweak observeables, and to A. Pomarol for useful discussions and for his comments on the manuscript.

\section{APPENDIX A: THE HIGGS SECTOR CUSTODIAL SYMMETRIES}

In this appendix we examine some symmetry aspects of the SGUT solutions, and in particular, of case (1). Some features of the latter, as well as the distinction between the two cases, can be better understood in terms of the custodial $S U(2)_{L+R}$ approximate symmetry of the SM lagrangian [26]; i.e., turning off hypercharge and flavor mixings, and if $h_{t}=h_{b}=h$, then one can rewrite the $t$ and $b$ Yukawa terms as an $S U(2)_{L} \times S U(2)_{R}$ invariant, i.e.,

$$
h\left(\begin{array}{c}
t_{L} \\
b_{L}
\end{array}\right)_{a} \epsilon_{a b}\left(\begin{array}{cc}
H_{1}^{0} & H_{2}^{+} \\
H_{1}^{-} & H_{2}^{0}
\end{array}\right)_{b c}\left(\begin{array}{c}
-b_{L}^{c} \\
t_{L}^{c}
\end{array}\right)_{c}
$$

(where in the $\mathrm{SM} H_{2}=i \tau_{2} H_{1}^{*}$ ) and $S U(2)_{L} \times S U(2)_{R} \rightarrow S U(2)_{L+R}$ for $\nu \neq 0$. However, $h_{t} \neq h_{b}$ and the different hypercharges of $t_{L}^{c}$ and $b_{L}^{c}$ explicitly break the left-right symmetry, and therefore the residual custodial symmetry.

In the MSSM, on the other hand, $H_{1}$ is distinct from $H_{2}$ and if $\nu_{\text {down }} \neq \nu_{u p} S U(2)_{L} \times$ $S U(2)_{R} \rightarrow U(1)_{T_{3 L}+T_{3 R}}$ [27. In particular, the $S U(2)_{L+R}$ symmetry is preserved if $\beta=\frac{\pi}{4}$ but is maximally broken if $\beta=\frac{\pi}{2}$. Ignoring hypercharge, we can then define for illustration two quantities,

$$
\begin{aligned}
B_{M} & \equiv \nu_{\text {down }} \frac{h_{t}-h_{b}}{m_{t}-m_{b}}, \\
B_{H} & \equiv m_{b} \frac{\tan \beta-1}{m_{t}-m_{b}},
\end{aligned}
$$

that measure the $S U(2)_{L+R}$ symmetry breaking in the matter and Higgs sectors, respectively. If we map the $\tan \beta-m_{t}^{\text {pole }}$ plane onto the $B_{M}-B_{H}$ plane, we find that cases (1) and (2) approximately correspond to $(1,0)$ and $(0,1)$, respectively. Thus, while the constrained $S O(10)$ solution realizes $m_{t} \neq m_{b}$ as an intrinsic property of the vacuum (i.e., $\nu_{u p} \gg \nu_{\text {down }}$ ) and the grand-unified symmetry protects $B_{M} \approx 0$ [29], the $\tan \beta \approx 1$ case realizes the $S U(2)_{L+R}$ (explicit) breaking as a scaling phenomenon (i.e., $B_{M} \propto h_{t}^{\text {fixed }}$ ) and the vacuum respects the symmetry (up to loop corrections). 
The symmetry that is spontaneously broken in case (2) is a $O_{4} \times O_{4}$ symmetry. Haber and Pomarol observed [28] that for $m_{3} \rightarrow 0$ [which is the situation in case (2)] there is no mixing between $H_{1}$ and $H_{2}$ and the Higgs sector respects $O_{4} \times O_{4}$ (up to gauge-coupling corrections). The symmetry is broken to $O_{3} \times O_{3}$ for $\nu_{\text {down }} \neq \nu_{u p} \neq 0$ and the six Goldstone bosons are the three SM Goldstone bosons, $A^{0}$, and $H^{ \pm}$. The symmetry is explicitly broken for $g_{2} \neq 0$ (so $m_{H^{+}}=M_{W}$ ) and is not exact even when neglecting gauge couplings (i.e., $m_{3} \neq 0$ ). Thus, $A^{0}$ and $H^{ \pm}$are massive pseudo-Goldstone bosons, i.e., $m_{H^{+}}^{2}-M_{W}^{2} \approx m_{A^{0}}^{2}=C \times m_{3}^{2}$. However, $C=-\frac{2}{\sin 2 \beta}$ and can be large, which is a manifestation of the fact that $O_{4} \times O_{4} \rightarrow O_{4} \times O_{3}$ for $\nu_{\text {down }}=0$. (The limit $m_{3} \rightarrow 0$ corresponds also to a $U(1)$ Peccei-Quinn symmetry [13].)

Our main interest in this work is, however, case (1), where from (9b) the Higgs-sector $S U(2)$ custodial symmetry constrains

$$
m_{H_{1}}^{2}+\mu^{2}=m_{H_{2}}^{2}+\mu^{2}=-m_{3}^{2} .
$$

This is realized by taking the limit $|\mu| \rightarrow \infty$, provided $|B| \approx|\mu|$ which in our formalism comes out as a prediction. (Indeed, many SGUT models can easily realize a large $B_{0}$, for example, see Ref. [52].) Relation (A3) results in a (tree-level) massless eigenvalue in the neutral CP-even mass matrix. (Note that the SM Goldstone bosons are also massless Goldstone bosons of the broken $S U(2) \times S U(2)$.)

The smallness of $\Delta_{\beta}$ is protected by the custodial symmetry, i.e., $\Delta_{\beta} \neq 0$ is explained by loop corrections. The broken symmetry in the Yukawa sector induces at the loop level a small breaking in the Higgs sector (e.g., $m_{H_{1}}^{2} \neq m_{H_{2}}^{2}$ ). The magnitude of the effect can be estimated by integrating $d \tan \beta / \tan \beta$, i.e.,

$$
\Delta_{\beta}\left(M_{Z}\right)=\Delta_{\beta}\left(M_{G}\right)-\frac{3}{16 \pi^{2}} \int_{\ln \frac{M_{G}}{M_{Z}}}^{0}\left(h_{t}^{2}-h_{b}^{2}\right) d \ln \frac{Q}{M_{Z}} \approx \Delta_{\beta}\left(M_{G}\right)+0.6,
$$

where we neglected $h_{\tau}$, as well as threshold corrections, took $h_{t} \approx 1, h_{b} \approx 0$, and assumed $\Delta_{\beta}<1$. Thus, even though the parameter space in case (1) is severely restricted, we can understand the restriction in terms of a symmetry.

\section{APPENDIX B: CONSTRAINTS ON COLOR AND CHARGE BREAKING MINIMA}

Let us consider the super-potential

$$
W=h_{t} \tilde{t}_{L} \tilde{t}_{R} H_{2}^{0}+\mu H_{1}^{0} H_{2}^{0},
$$

where we have performed an $S U(2)$ rotation so that $H_{2}^{+}$has no vacuum expectation value. (We do not distinguish our notation for a superfield from that of its scalar component.) It is easy to convince oneself that only those terms in the super-potential are relevant when searching for the global minimum of the scalar potential in the large $h_{t}$ limit [53]. Using standard techniques we arrive at the corresponding scalar potential:

$$
h_{t}^{2} V=M^{2}-\Gamma+\Lambda,
$$

where the bilinear, trilinear, and quartic terms are 


$$
\begin{gathered}
M^{2}=m_{1}^{2} H_{1}^{0^{2}}+m_{2}^{2} H_{2}^{0^{2}}+2 m_{3}^{2} H_{1}^{0} H_{2}^{0}+m_{\tilde{t}_{L}}^{2} \tilde{t}_{L}^{2}+m_{\tilde{t}_{R}}^{2} \tilde{t}_{R}^{2}, \\
\Gamma=\left|2\left(\left|A_{t}\right| H_{2}^{0}+s|\mu| H_{1}^{0}\right) \tilde{t}_{L} \tilde{t}_{R}\right|
\end{gathered}
$$

and

$$
\Lambda=\left(\tilde{t}_{L}^{2}+\tilde{t}_{R}^{2}\right) H_{2}^{02}+\tilde{t}_{L}^{2} \tilde{t}_{R}^{2}+\frac{1}{h_{t}^{2}}\left(\frac{\pi}{2} \times \text { “D - terms" }\right),
$$

respectively. All fields were scaled $\phi \rightarrow \phi / h_{t}$ and are taken to be real and positive (our phase choice for the fields, which fixed $m_{3}^{2}<0$ and $\Gamma>0$, i.e., maximized the negative contributions to $V$ ) and all parameters are real. $m_{1,2}^{2}=m_{H_{1,2}}^{2}+\mu^{2}, s=\mu A_{t} /\left|\mu A_{t}\right|$, and the expression for the " $D$-terms" is [53]

$$
\frac{12}{5} \alpha_{1}\left[-\frac{H_{1}^{0^{2}}}{2}+\frac{H_{2}^{0^{2}}}{2}+\frac{\tilde{t}_{L}^{2}}{6}-\frac{2 \tilde{t}_{R}^{2}}{3}\right]^{2}+\alpha_{2}\left[H_{1}^{0^{2}}-H_{2}^{0^{2}}+\tilde{t}_{L}^{2}\right]^{2}+\frac{4}{3} \alpha_{3}\left[\tilde{t}_{L}^{2}-\tilde{t}_{R}^{2}\right]^{2} .
$$

We can parametrize the four fields in terms of an overall scale $X$ and three angles $0 \leq$ $\alpha, \beta, \gamma \leq \frac{\pi}{2}: H_{1}^{0}=X \sin \alpha \cos \beta, H_{2}^{0}=X \sin \alpha \sin \beta, \tilde{t}_{R}=X \cos \alpha \cos \gamma, \tilde{t}_{L}=X \cos \alpha \sin \gamma$, and redefine

$$
h_{t}^{2} V(X)=M^{2}(\alpha, \beta, \gamma) X^{2}-\Gamma(\alpha, \beta, \gamma) X^{3}+\Lambda(\alpha, \beta, \gamma) X^{4}
$$

Then, for fixed angles, $V(X)$ will have a minimum for $X \neq 0$ provided the condition $32 M^{2} \Lambda<9 \Gamma^{2}$ is satisfied. In that case,

$$
X_{\text {min }}=\frac{3}{8} \frac{\Gamma}{\Lambda}\left[1+\left(1-\frac{32 M^{2} \Lambda}{9 \Gamma^{2}}\right)^{\frac{1}{2}}\right] \geq 0
$$

and

$$
h_{t}^{2} V_{\min }=-\frac{1}{2} X_{\min }^{2}\left(\frac{\Gamma}{2} X_{\min }-M^{2}\right)
$$

We only allow parameters for which a standard model (SM) minimum exists. The SM minimum corresponds to $\alpha=\frac{\pi}{2}$ and $\beta=\beta^{0}\left(\gamma\right.$ is irrelevant and $\tan \beta^{0}=\nu_{\text {up }} / \nu_{\text {down }}$ is the angle used to fix $\mu, m_{3}$, as well as the Yukawa couplings). It is easy to convince oneself that in that limit the $4 \times 4$ second-derivative matrix is $2 \times 2$ block diagonal (otherwise baryon number is violated). Thus, it is sufficient to confirm that the four physical eigenvalues are positive to ensure that it is a minimum. (This is done in our numerical calculations.) If these conditions are satisfied then the SM is at least a local (negative-energy) minimum, and one has $\Gamma_{\mathrm{SM}}=0, M_{\mathrm{SM}}^{2}<0$, and $(\mathrm{B} 8)$ and $(\mathbb{B} 9)$ reduce to the usual results $X_{\text {min }}^{\mathrm{SM}}=\sqrt{-M_{\mathrm{SM}}^{2} / 2 \Lambda_{\mathrm{SM}}}$, $h_{t}^{2} V_{\text {min }}^{\mathrm{SM}}=-M_{\mathrm{SM}}^{4} / 4 \Lambda_{\mathrm{SM}}$.

Let us now consider the possibility of additional CCB minima with $\cos \alpha \neq 0$. Since $m_{\tilde{t}_{L, R}}^{2}>0$ for the class of models we are considering this requires $\Gamma \neq 0$, which we assume hereafter. From (B/ $\mathrm{B} 7)$ - (B9) it is easy to classify the possible color and/or charge breaking (CCB) minima for definite $\alpha, \beta, \gamma$. One finds that for $\Gamma^{2} \leq 32 \Lambda M^{2} / 9$ there is no CCB 
minimum, while for $32 \Lambda M^{2} / 9<\Gamma^{2} \leq 4 \Lambda M^{2}$ the CCB minimum exists but has $V_{\min }^{\mathrm{CCB}}>0>$ $V_{\text {min }}^{\text {SM }}$, which is presumably safe. For $4 \Lambda M^{2}<\Gamma^{2}$ (including the more rare case $M^{2}<0$, that must fall in this category) there is a negative-value CCB minimum, which may however be either local (presumably safe), i.e., $V_{\text {min }}^{\mathrm{CCB}}>V_{\text {min }}^{\mathrm{SM}}$, or global (probably unacceptable), i.e., $V_{\text {min }}^{\mathrm{CCB}}<V_{\text {min }}^{\mathrm{SM}}$. Here, a sufficient (but not necessary) condition for an acceptable model is

$$
\Gamma^{2} \leq 4 \Lambda M^{2}
$$

which generalizes that of Ref. [53]. In principle, the above discussion holds for any number of fields (i.e., any number of angles), only the explicit expressions for $M^{2}, \Gamma$, and $\Lambda$ are more complicated. If constraint (B10) holds for every choice of $\alpha, \beta$, and $\gamma(\cos \alpha \neq 0)$ then there is no negative-valued color and/or charge breaking minimum, global (GCCB) or local. In the special case $M^{2}<0$ the constraint cannot be satisfied and there will be a negative-energy local CCB minimum, an observation made previously by Gunion et al. [53].

If (B10) does not hold, further investigation is needed to determine whether the minimum is global or local. It is straightforward to show that (B10) is satisfied for $\sin \beta=0$, i.e., there is no negative-valued CCB minimum for $H_{2}^{0}=0$ for the class of models we are considering. We will therefore restrict our attention to the case $H_{2}^{0} \neq 0$, in which case it is convenient to reparametrize $X=H_{2}^{0}$ and rescale all fields by an additional factor of $1 / H_{2}^{0}$. This simplifies the discussion and enables us to examine and generalize previous work on the subject 54,43,55,53.

By inspection (B10) cannot hold for all values of the fields and there is always (and not just for $M^{2}<0$, which is hard to achieve in dangerous directions) a local CCB minimum, e.g., for $M^{2}=0$ ( $\Lambda$ is positive definite). For example, one can choose

$$
\begin{gathered}
H_{1}^{0}=-\frac{m_{3}^{2}}{m_{1}^{2}}, \\
\tilde{t}_{L}=\frac{1}{m_{\tilde{t}_{L}}} \sqrt{\frac{1}{2}\left(\frac{m_{3}^{4}}{m_{1}^{2}}-m_{2}^{2}\right),} \\
\tilde{t}_{R}=\tilde{t}_{L} \frac{m_{\tilde{t}_{L}}}{m_{\tilde{t}_{R}}},
\end{gathered}
$$

which is always possible because of (8). We confirmed this in our numerical studies, i.e., there is a CCB (negative-valued) local minimum even if $V^{\mathrm{SM}}=V\left(\nu_{\text {down }}, \nu_{u p}\right)$ is the global minimum. (We do not consider additive constants.) Although such local minima may be of cosmological interest, the relevant question in our case is whether $V^{\mathrm{SM}}$ is the global minimum of the full (or more precisely, of the four-field) scalar potential. Similarly, we assume that positive-valued CCB minima would not be populated and are therefore harmless. The answer has to be given by numerical mapping of all minima, but it is still useful to review how one could derive analytic constraints from (B10), which are typically relevant only for specific regions of the parameter space.

For example, if we fix $H_{1}^{0}=0, \tilde{t}_{L}=\tilde{t}_{R}=1$, then (B10) gives the well known result [54]

$$
A_{t}^{2} \leq 3\left(m_{\tilde{t}_{L}}^{2}+m_{\tilde{t}_{R}}^{2}+m_{H_{2}}^{2}+\mu^{2}\right)
$$


The equal field direction was chosen so the positive $D$-term contribution $\propto 1 / h_{t}^{2}$ vanishes. This is not a relevant requirement for $h_{t} \approx 1$ (i.e., there may exist a deeper minimum with non vanishing $D$-terms). It is obvious that (B12) is not relevant when $|\mu| \rightarrow \infty$. We find that a more useful constraint is [taking $H_{1}^{0} \approx 1, \tilde{t}_{L} \approx \tilde{t}_{R} \equiv t \ll 1$ and using (Bत10)]

$$
\left(\left|A_{t}\right|+s|\mu|\right)^{2} \leq 2\left(m_{\tilde{t}_{L}}^{2}+m_{\tilde{t}_{R}}^{2}\right) .
$$

Note that if the order $t^{2}$ corrections to (B13) are not negligible then $V$ is less negative, which motivated our choice $t \ll 1$, i.e., the more dangerous direction. That constraint was also used by Drees et al. [43] to describe their numerical results (i.e., if we take $m_{1}=m_{2}$ in their formula). We confirmed in our numerical studies that (B13) and not (B12) is relevant in our case. [We found nearly all points to be consistent with (B12) for our ranges of the soft parameters.]

Finally, we perform numerical minimization of the potential (B2) and map all minima using monte-carlo routines. We find that $(\overline{B 13})$ is to a good approximation sufficient to avoid a GCCB minimum, but is not necessary. For example, only about $80 \%$ (15\%) of the points which are inconsistent with (B13) correspond to GCCB for $m_{t}^{\text {pole }} \lesssim 165 \mathrm{GeV}$ $\left(m_{t}^{\text {pole }} \gtrsim 165 \mathrm{GeV}\right)$. We compare the points consistent with $(\mathrm{B13})$ and with the numerical search in Fig. 8. The greater relevance of (B13) for $m_{t}^{\text {pole }} \lesssim 165 \mathrm{GeV}$ can be understood if we recall that the lower bound on $|\cos 2 \beta|$ increases with $m_{t}^{\text {pole }}$, and thus $V^{\text {sm }}$ becomes a deeper minimum. (The lower bound is related to $h_{t}$ unitarity, and that statement is independent of our $h_{b}=h_{\tau}$ assumption.) On the other hand, $|\mu|$ and the negative terms in $V^{\mathrm{CCB}}$ [e.g., Eq. (B2)] diminish with increasing $m_{t}^{\text {pole }}$. That interplay shifts the CCB minimum from a global to a local minimum.

Let us demonstrate that claim in a simple-minded example assuming $h_{t} \approx h_{t}^{\text {fixed }} \approx 1-1.1$. We define $\sin ^{2} \beta=2 m_{t}^{2} / h_{t}^{2} \nu^{2} \equiv x, 0.5 \leq x \leq 1\left[\nu=2 M_{W} / g_{2}\right]$. We can rewrite

$$
h_{t}^{2} V^{\mathrm{SM}}=-\frac{1}{8} M_{Z}^{2} h_{t}^{2} \nu^{2}[1-4 x(1-x)] .
$$

$\left|h_{t}^{2} V^{\mathrm{SM}}\right|$ sharply increases with $m_{t}^{\text {pole }}$ (i.e., with $x$ ). On the other hand, taking $m_{H_{2}}^{2} \approx M_{Z}^{2} \approx 0$ in (9a) we have

$$
\mu^{2} \approx m_{H_{1}}^{2}\left(\frac{1-x}{2 x-1}\right),
$$

and $\mu^{2}$ decreases from $+\infty$ to 0 in the above range of $x$. Now assume that constraint (B13) is not satisfied, i.e.,

$$
h_{t}^{2} V^{\mathrm{CCB}} \approx-\frac{1}{2} \kappa^{2} \mu^{2}\left(\kappa \mu^{2}-m_{\tilde{t}_{L}}^{2}-m_{\tilde{t}_{R}}^{2}\right) t^{2},
$$

where $t^{4} \ll t^{2}$ and $\kappa$ is a positive number in the range $\left[\frac{3}{8}, \frac{3}{4}\right]\left(X_{\min }=\kappa|\mu|\right)$. We also assumed $\left|A_{t}\right| \ll|\mu|$, and neglected $t^{4}$ terms. $\left(M^{2}<0\right.$ would increase $\kappa$.) To further simplify, consider the case $m_{0}=0$, so that $m_{\tilde{t}_{L}}^{2} \approx m_{\tilde{t}_{R}}^{2} \approx 6 M_{\frac{1}{2}}^{2} \approx 12 m_{H_{1}}^{2} \cdot V^{\mathrm{CCB}} \lesssim V^{\mathrm{SM}}$ in this case if

$$
\frac{1}{4 \kappa^{2}} \frac{M_{Z}^{2} h_{t}^{2} \nu^{2}}{m_{H_{1}}^{4} t^{2}} \lesssim\left(\frac{1-x}{2 x-1}\right) \frac{\left[\kappa\left(\frac{1-x}{2 x-1}\right)-24\right]}{1-4 x(1-x)} .
$$


The r.h.s. has to be positive, i.e., $x \rightarrow 0.5^{+}$or $m_{t}^{\text {pole }} \lesssim 150 \mathrm{GeV}$. Slightly increasing $m_{t}^{\text {pole }}$ would reverse the inequality.

Lastly, there are two caveats. The first is that we used in this section the (one-loop improved) tree-level potential. However, following Ref. [49] we perform the calculations at the $t$-scalar scale to eliminate large loop corrections. Secondly, a GCCB minimum may be "safe" if separated from the local standard-model minimum by a tunneling time greater than the age of the universe [50]. Such considerations are beyond the scope of our present work and would also require a consideration of finite-temperature effects.

\section{APPENDIX C: CALCULATION OF THE LOOP-INDUCED MASS}

\section{The EPM: Run and diagonalize}

A straightforward way to calculate $\Delta_{h^{0}}$ is $(a)$ to take the first and second derivatives of $\Delta V(Q)$ with respect to the neutral CP-even and CP-odd components of $H_{i}$ at the minimum; (b) to absorb the latter in $m_{A^{0}}$; and (c) to calculate the correction to (11): the effective potential method (EPM). Like Ref. [44, we will follow Ref. [45], however, we add to their expressions "D-term" [46] and Higgs-Higgsino and gauge-gaugino [36] contributions, which are typically $-(1-2)$ and $-(2-4) \mathrm{GeV}$, respectively. Note that $(i)$ all the parameters are taken at the subtraction scale $Q$. The mass matrices are thus calculated at the scale $Q$ and (ii) only then are diagonalized, i.e., a "run and diagonalize" algorithm. Our two

choices in section $\mathbb{\square}$ were $Q=M_{Z}$ and $Q=600 \mathrm{GeV} \sim m_{\tilde{t}}$. (Large $\ln \frac{m_{A^{0}}}{M_{Z}}$ logarithms are multiplied by small couplings and do not invalidate the loop expansion, i.e., the EPM. This is equivalent to the statement above that Higgs-Higgsino contributions are small.) One corrects for threshold effects by the subtraction of one-loop leading logarithms (included in $\Delta V$ ), and thus to that order the two choices are equivalent.

The $Q$ dependence of $m_{h^{0}}(Q)$ comes about from $(i)$ logarithmic wave-function dependence of $\nu_{i}(Q)$ and (ii) two-loop implicit field-independent dependences of $\Delta V(Q)=$ $\Delta V\left[m_{i}(Q), \alpha_{i}(Q), h_{t}(Q)\right]$ (where $m_{i}$ runs over the relevant mass parameters). Both are often underestimated. The importance of $(i)$ is obvious, e.g., from the $m_{t}^{4} / M_{Z}^{2}$ factor. The field-independent dependence (ii) was studied in Ref. [56], where it was shown to be of the order of two-loop (next-to-leading) logarithms. One can instead examine directly the expression for $\Delta_{h^{0}}$, e.g., the leading logarithm in (14) evolves with scale (neglecting $\Delta_{\theta_{t}}$ and multiplicative factors) roughly as

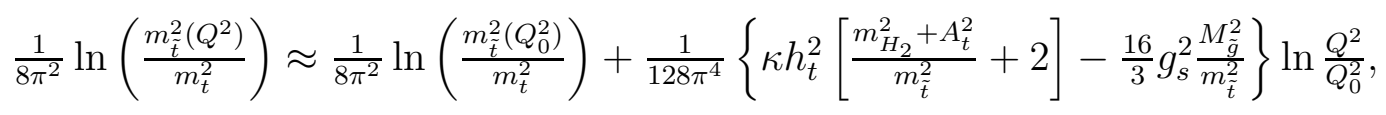

where $\kappa=1,2$ for $\tilde{t}_{L, R}$, respectively, and we took $m_{t}(Q) \approx m_{t}\left(Q_{0}\right)$, etc., $m_{\tilde{t}_{L}} \approx m_{\tilde{t}_{R}}$, and neglected all terms aside from $h_{t}$ and $g_{s}^{2}\left(=4 \pi \alpha_{s}\right)$ ones. ( $M_{\tilde{g}}$ is the gluino mass.) This is of course a rough estimate, as, in practice all the parameters scale with $Q$. However, it illustrates qualitatively the presence of residual two-loop logarithms which are enhanced by the large couplings and masses when evaluating $\Delta_{h^{0}}$. Note that typically the QCD term wins and the correction is positive near $Q=M_{Z}$.

Though formally of two-loop order, the residual $Q$ dependence of the EPM $m_{h^{0}}$ is (in our case) of order $10-20 \%$. The ambiguity in $m_{h^{0}}$ is reduced for $m_{h^{0}}^{T} \gg 0$. Also, for values 
of $\tan \beta$ outside the allowed region $\Delta_{\theta_{t}}$ is diminished (e.g., in the notation of Ref. [45] only $\Delta_{22}$ is important), $h_{t}<1$ (which also diminishes the wave-function renormalization), and thus, there is a smaller ambiguity not only in $m_{h^{0}}$ but also in $\Delta_{h^{0}}$. Nevertheless, one should be aware of such ambiguities, which are generically present in a calculation of that sort.

\section{The RGM: Diagonalize and run}

One could alternatively correct for thresholds by explicitly decoupling a particle below its threshold and redefining the effective field theory: the renormalization group method (RGM). Following Ref. [36,47], we diagonalize the Higgs mass matrices at scale $Q=m_{A^{0}}$, integrate out the heavy Higgs doublet, and define a one Higgs doublet model (1HDM) effective theory with a quartic coupling $\frac{\lambda}{2} h^{4}$. Note that $m_{A^{0}} \gg m_{\tilde{t}}$, so only the heavy Higgs doublet is integrated out at $Q=m_{A^{0}}$, and other heavy particles will be decoupled at lower thresholds. The boundary condition for $\lambda\left(m_{A^{0}}\right)$ is determined by the MSSM Higgs couplings at that scale and requires knowledge of $\tan \beta\left(m_{A^{0}}\right)$ (i.e., wave-function renormalization). Mixing effects are accounted for by a complicated set of matching conditions 44, 47]. $\lambda$ is then evolved down to $M_{Z}$, where $m_{h^{0}}=\sqrt{\lambda} \nu=2 \sqrt{\lambda} M_{W} / g_{2}$; i.e., a "diagonalize and run" algorithm.

One can solve the RGE's iteratively to get a semi-analytic approximation [36,47]. This is not an exact procedure, and at one-loop is accurate to leading logarithms (i.e., the accuracy of the EPM). Alternatively, if the RGM calculation is done by exact (or numerical) integration, then the leading logarithms are summed to all orders. In agreement with Ref. 36. we find that $\lambda\left(M_{Z}\right)$ (and thus $m_{h^{0}}$ ) is diminished when integrating the RGE's numerically. However, the effect of the numerical integration (Fig. 5) is more important than anticipated in Ref. 36] since $A^{0}$ is heavier than the mass-scale used there and because of the large couplings. The difference between the numerical treatment and the approximate formula is of order of two-loop (leading) logarithms. The summation of the leading logarithms modifies the scale dependence of the calculated mass.

A different scale dependence is via the details of the decoupling of heavy states. To ease the calculation, we do not diagonalize scalar-quark mass-squared matrices at an intermediate scale. Instead, we put in the appropriate matching conditions 48,47 by hand. These conditions are derived by expanding the EP assuming $\left|m_{t} \mu\right| \lesssim m_{\tilde{t_{L}}}^{2}$, etc., which still holds in our case $\left[m_{t}\left(m_{A^{0}}\right)<m_{t}\left(M_{Z}\right)\right]$. Nevertheless, we repeated the 1HDM RGM calculation but using the EPM with $Q \approx m_{A^{0}}$ to determine $m_{h^{0}}\left(m_{A^{0}}\right)$, and thus [using $\nu\left(m_{A^{0}}\right)$ ] the boundary condition for $\lambda\left(m_{A^{0}}\right)$. The agreement with the calculation using the boundary conditions of Ref. 48,47 is good.

\section{Validity of the comparison between the EPM and RGM}

Whether "running and diagonalizing" or vice versa leads to a slightly different answer. However, it was already noted in Ref. [36] that the difference between the two algorithms is small and of higher order, and can be ignored for the purpose of our discussion here. However, the inclusion of the appropriate RGM matching conditions is essential for the comparison between the two methods. 
More importantly, the RGM approximation of 1HDM breaks down if $|\mu|$ is not sufficiently large. (In such cases the more conventional 2HDM RGM is appropriate.) Too small scalarquark (diagonal) masses break down the expansion of Ref. [48,47] and too large scalar threshold-corrections can break down the EPM approximation. Indeed, one has to ensure that the scalar quarks are not heavier than about $1 \mathrm{TeV}$ in order not to invalidate the loop expansion which underlies the EP, as these large logarithms would be multiplied by large coupling: where the RGM and EPM are in sharp disagreement (e.g., in Figs. 5 and 6) correspond to either one of these cases.

\section{Two-loop calculations}

In this work we do not carry out a two-loop calculation of $\Delta_{h^{0}}$. Such a calculation is an elaborate task and attention should be paid to various issues, e.g., wave-function renormalization and the coupled running of the masses and couplings; pole masses vs. running masses; the multi-scale structure of the low-energy theory; the correct choice of matching conditions (logarithmic and non-logarithmic) given a choice of decoupling scales; one-loop finite pieces (not included in the EP) that may become important in certain regions of the parameter space; in addition to correct counting of powers of the couplings. In fact, an hybrid algorithm between the EPM and RGM is required.

We received recently two interesting papers where (partial) two-loop analyses were presented [58,59]. In this section we compare those results with the residual two-loop terms we identified above. We will take a somewhat critical point of view emphasizing that though the above studies (as well as the discussion here) pave the way to understanding $\Delta_{h^{0}}$ beyond one-loop order, they are not complete. We conclude that the one-loop leading-logarithm result is most probably an upper bound on the two-loop result, but we doubt whether any stronger conclusions can be drawn at the present.

Above, we presented the leading-logarithm result. In the EPM this is given by $\Delta V^{\text {one-loop }}$ and in the RGM one solves the RGE's to that order. We found no significant difference between the two methods. Next, we proceeded to calculate beyond the leading order and integrated the RGM RGE's numerically, summing the leading logarithms to all orders in perturbation theory, and, in particular, to two-loop order. We then found a $\sim-10-20 \%$ correction to $\Delta_{h^{0}}$. On the other hand, we identified in the EPM expressions next-to-leading two-loop logarithmic terms. Those terms come about from the scale dependence of the running parameters, and introduce a similar (but positive) correction to $\Delta_{h^{0}}$. Also, we noted the important $(\sim 10 \%)$ effect of $m_{t}^{\text {pole }} / m_{t}^{\text {running }} \approx\left[1+\left(4 \alpha_{s} / 3 \pi\right)\right.$ ] [e.g., $\left(m_{t}^{\text {running }}\right)^{4} \approx$ $\left.0.8\left(m_{t}^{\text {pole }}\right)^{4}\right]$. It is then suggestive that the two-loop leading logarithms and $m_{t}$ corrections would diminish the one-loop (leading-logarithm) result, while the next-to-leading logarithms would partially counter-balance that effect. Thus, we reach the conclusion that the one-loop leading-logarithm prediction for $m_{h^{0}}$ (which contain some next-to-leading logarithms) is an upper bound on the two-loop prediction. Any stronger conclusion would require a detailed

\footnotetext{
${ }^{14}$ This requirement is consistent with our choice of ranges for the soft parameters.
} 
consideration of the issues listed above. Furthermore, the significance of a detailed twoloop study in the context of SGUT's is not clear unless complemented by similar studies of threshold effects near the high-scale boundary.

Let us proceed with the issue of the orders in perturbation theory. It was already pointed out by Chankowski [57] that the inclusion of $\Delta V^{\text {one-loop }}$ in (7) with only one-loop RGE's for the (RG-improved) potential parameters is an inconsistent procedure when counting powers of couplings. These issues have recently received great attention in the context of theories with a single scalar field (i.e., $\phi^{4}$ or the SM) 60, 61]. It was explicitly shown that a RGimproved L-loop effective potential is consistent when the parameters are calculated using $(\mathrm{L}+1)$-loop RGE's 61.

The observations of Ref. [60,61] were recently applied to the MSSM by Kodaira et al. [58] who extended a previous work of Espinosa and Quiros 662. Indeed, they find that the two-loop RGM with one-loop EP slightly increase the one-loop RGM result. However, they impose the naive assumption of only one relevant scale $\left(M_{S U S Y}\right)$ below which the SM RGE's are in effect (and the EP contain only a $m_{t}$ correction). Thus, the MSSM parameters are not treated on the same footing as the SM ones, and one expects some modification of their numerical results. However, we note that the correction is positive: recall that the difference between the two RGM approximations is the next-to-leading logarithms, which we found to be positive by examining the scale dependence of the result.

Hempfling and Hoang [59] explicitly calculate the two-loop RGM correction (using the methods of Ref. [47]), finding

$$
\delta \Delta_{h^{0}}^{2} \approx \frac{3}{\left(16 \pi^{2}\right)^{2}} 8 \sqrt{2} G_{\mu} m_{t}^{4} \ln \frac{M_{S U S Y}^{2}}{m_{t}^{2}}\left[\frac{16}{3} g_{s}^{2}-5 h_{t}^{2}\right],
$$

which roughly agrees with our Eq. (C1).

Those authors go further to derive a diagramatic two-loop result (in a simplified model) which diminishes $\Delta_{h^{0}}$ below its two-loop RGM prediction. They then show that, e.g., if the difference between the pole and running $t$-quark mass is accounted for in the RGM calculation by appropriate matching conditions, the two methods agree. This again stresses the need for adequate choice of matching conditions. However, it is not clear that there are no other effects of that order of magnitude that can slightly increase $\Delta_{h^{0}}$. [Also, we showed that scalar mixings which are neglected in the above two-loop calculations and in our Eq. (C1), are not apriori negligible.]

In short, all analyses support the conclusion that two-loop logarithms will diminish the one-loop leading logarithm result (in particular, if one properly distinguishes the $t$-quark pole mass from the running mass). However, it is not clear that the prediction of a complete two-loop analysis lies as low as suggested in Ref. [59] (or by our Fig. 10). (If indeed this is the case our upper bounds will be significantly strengthened.)

\section{APPENDIX D: EXTENDED HIGGS SECTORS}

If there is an extended heavy Higgs sector (as is assumed by some authors when trying to explain the light fermion spectrum) with large representations coupling to the third family (i.e., there is no flavor symmetry that forbids such couplings) then (2) does not hold and our 
constraints are evaded. Alternatively, some models extend the light Higgs sector by adding a SM singlet superfield, $S$, to the spectrum. This is the only addition to the Higgs sector which is consistent (i.e., without fine-tuned cancellations) with coupling constant unification.

The new singlet(s) would mix with the other neutral Higgs bosons and would thus contribute to $m_{h^{0}}^{T}$; e.g., [63],

$$
m_{h^{0}}^{T^{2}} \leq M_{Z}^{2} \cos ^{2} 2 \beta+\lambda_{s}^{2} \frac{\nu^{2}}{2} \sin ^{2} 2 \beta,
$$

where $\lambda_{s}\left(\kappa_{s}\right)$ is a new Yukawa coupling in the superpotential: $\lambda_{s} H_{1} H_{2} S\left(\kappa_{s} S^{3}\right)$. (We do not distinguish here our notation of superfields from that of their scalar components.) The MSSM limit with $m_{h^{0}}^{T}=0$ is recovered if $\lambda_{s} \rightarrow 0$, which is indeed the case if $h_{t} \approx h_{t}^{\text {fixed }}$ 64]. However, the new Yukawa couplings (i.e., $\lambda_{s} \neq 0$ ) enable one to realize (2) for somewhat smaller values of $h_{t}<h_{t}^{\text {fixed }}$ (and $\tan \beta>1$ ). The shift in the allowed region is shown in Fig. 11 for $\lambda_{s}=0.5$ and $\kappa_{s}=0$. Thus, $m_{h^{0}}^{T}$ can be large and $m_{h^{0}}$ can be heavy $\sim 150 \mathrm{GeV}$ [19,20.64 when loop corrections are added. (See also Ref. [65].) 


\section{REFERENCES}

[1] H. Georgi and S. L. Glashow, Phys. Rev. Lett. 32, 438 (1974); H. Georgi, H. R. Quinn, and S. Weinberg, Phys. Rev. Lett. 33451 (1974); E. Witten, Nucl. Phys. B188, 513 (1981); S. Dimopoulos and H. Georgi, ibid. 193, 150 (1981); N. Sakai, Z. Phys. C 11, 153 (1981); S. Dimopoulos, S. Raby, and F. Wilczek, Phys. Rev. D 24, 1681 (1981); W. J. Marciano and G. Senjanovic, ibid. 25, 3092 (1982); L. E. Ibanez and G. G. Ross, Phys. Lett. 105B, 439 (1982); M. B. Einhorn and D. R. T. Jones, Nucl. Phys. B196, 475 (1982).

[2] For reviews, see P. Langacker, Phys. Rep. 72, 185 (1981); in Proceedings of the Ninth Workshop on Grand Unification, Aix les Bains, France, 1988, ed. R. Barloutaud (World Scientific, Singapore, 1988) p.3; H. P. Nilles, Phys. Rep. 110, 1 (1984); in Testing The Standard Model, ed. M. Cvetič and P. Langacker (World Scientific, Singapore, 1991) p. 633; H. E. Haber and G. L. Kane, Phys. Rep. 117, 75 (1985). See also P. Nath et al., Applied N=1 Supergravity (World Scientific, Singapore, 1984); G. G. Ross, Grand Unified Theories (Benjamin, New York, 1984); R. N. Mohapatra, Unification and Supersymmetry (Springer, New York, 1986, 1992). For recent reviews, see, for example, H. E. Haber, Santa Cruz Report No. SCIPP 92/33 (1993); J. L. Lopez, Texas A\&M Report No. CTP-TAMU-42/93 (1993); R. Arnowitt and P. Nath, Texas A\&M Report No. CTP-TAMU-52/93 (1993).

[3] M. S. Chanowitz, J. Ellis, and M. Gaillard, Nucl. Phys. B128, 506 (1977); A. J. Buras, J. Ellis, M. K. Gaillard, and D. V. Nanopoulos, ibid. 135, 66 (1978).

[4] P. Langacker and M. Luo, Phys. Rev. D 44, 817 (1991); J. Ellis, S. Kelley and D. V. Nanopoulos, Phys. Lett. B249, 441 (1990); U. Amaldi, W. de Boer and H. Fürstenau, ibid. 260, 447 (1991); F. Anselmo, L. Cifarelli, A. Peterman and A. Zichichi, Nouvo Cimento 104A, 1817 (1991).

[5] B. Ananthanarayan, G. Lazarides and Q. Shafi, Phys. Rev. D 44, 1613 (1991); M. Bando, T. Kugo, N. Maekawa and H. Nakano, Mod. Phys. Lett. A 7, 3379 (1992); H. Arason et al., Phys. Rev. Lett. 67, 2933 (1991); A. Giveon, L. J. Hall and U. Sarid, Phys. Lett. B271, 138 (1991); S. Kelley, J. L. Lopez, and D. V. Nanopoulos, ibid. 274, 387 (1992).

[6] P. Langacker and N. Polonsky, Phys. Rev. D 47, 4028 (1993).

[7] G. Degrassi, S. Fanchiotti, and A. Sirlin, Nucl. Phys. B351, 49 (1991).

[8] P. Langacker and N. Polonsky, Phys. Rev. D 49, 1454 (1994).

[9] M. Dine, R. Leigh, and A. Kagan, Phys. Rev. D 48, 4269 (1993); Y. Nir and N. Seiberg, Phys. Lett. B309, 337 (1993); P. Pouliot and N. Seiberg, ibid. 318, 169 (1993).

[10] V. Barger, M. S. Berger, and P. Ohmann, Phys. Rev. D 47, 1093 (1993).

[11] M. Carena, S. Pokorski, and C. E. M. Wagner, Nucl. Phys. B406, 59 (1993); W. A. Bardeen, M. Carena, S. Pokorski, and C. E. M. Wagner, Max Planck Report No. MPIPH-93-58 (1993).

[12] V. Barger, M. S. Berger, P. Ohmann, and R. J. Phillips, Phys. Lett. B314, 351 (1993).

[13] L. J. Hall, R. Rattazzi, and U. Sarid, LBL Report No. LBL-33997 (1993).

[14] G. L. Kane, C. Kolda, L. Roszkowski, and J. D. Wells, private communications and Michigan Report No. UM-TH-93-24 (1993).

[15] N. Polonsky, in proceedings of the International Workshop on Supersymmetry and Uni- 
fication of Fundamental Interactions, Boston, MA, 1993, ed. P. Nath (World Scientific, Singapore, 1993) p. 398.

[16] N. Polonsky, Pennsylvania Report No. UPR-0588T (1993).

[17] P. Langacker, in Recent Directions in Particle Theory, ed. J. Harvey and J. Polchinski (World Scientific, Singapore, 1993) p. 141.

[18] For reviews, see M. Sher, Phys. Rep. 179, 273 (1989); J. F. Gunion, H. E. Haber, G. Kane, and S. Dawson, The Higgs Hunter's Guide (Addison-Wesley, Reading, 1990).

[19] J. R. Espinosa and M. Quiros, Phys. Lett. B302, 51 (1993).

[20] G. L. Kane, C. Kolda, and J. D. Wells, Phys. Rev. Lett. 70, 2686 (1993).

[21] M. Bando, T. Kugo, N. Maekawa and H. Nakano, Mod. Phys. Lett. A 7, 3379 (1992); M. Bando, N. Maekawa, H. Nakano, and J. Sato, ibid. 8, 2729 (1993).

[22] See, for example, J. E. Björkman and D. R. T. Jones, Nucl. Phys. B259 533 (1985); N. K. Flack, Z. Phys. C 30, 247 (1986); M. Drees and M. M. Nojiri, Nucl. Phys. B369, 54 (1992). See also Ref. [10, 38,57,477, as well as D. J. Castano, E. J. Piard, and P. Ramond, Florida Report No. UFIFT-HEP-93-18.

[23] K. Inoue, A. Kakuto, H. Komatsu, and S. Takeshita, Prog. Theor. Phys. 67, 1889 (1982).

[24] S. Coleman and E. Weinberg, Phys. Rev. D 7, 1888 (1973); S. Weinberg, ibid. 2887 (1973).

[25] R. Arnowitt and P. Nath, Phys. Rev. D 46, 3981 (1992).

[26] S. Weinberg, Phys. Rev. D 19, 1277 (1979); L. Susskind, ibid. 20, 2619 (1979); P. Sikivie et al., Nucl. Phys. B173, 189 (1980).

[27] R. Barbieri and L. Maiani, Nucl. Phys. B244, 32 (1983).

[28] H. E. Haber and A. Pomarol, Phys. Lett. B302, 435 (1993).

[29] M. Olechowski and S. Pokorski, Phys. Lett. B214, 393 (1988); Nucl. Phys. B404, 590 (1993).

[30] B. Ananthanarayan, G. Lazarides, and Q. Shafi, Phys. Lett. B300, 245 (1993); B. Ananthanarayan and Q. Shafi, Bartol Report No. BA-93-25 (1993).

[31] M. Carena, M. Olechowski, S. Pokorski, and C. E. M. Wagner, CERN Report No. CERN-TH-7163-94 (1994).

[32] A. E. Nelson and L. Randall, Phys. Lett. B316, 516 (1993).

[33] M. Boulware and D. Finnel, Phys. Rev. D 442054 (1991).

[34] C. T. Hill, Phys. Rev. D 24, 691 (1981). See also M. Cvetič and C. R. Preitschopf, Nucl. Phys. B272, 490 (1986).

[35] E. Franco and A. Morelli, Nouvo Cimento 96A, 257 (1986).

[36] M. A. Diaz and H. E. Haber, Phys. Rev. D 46, 3086 (1992).

[37] M. Carena, M. Olechowski, S. Pokorski, and C. E. M. Wagner, CERN Report No. CERN-TH-7060-93 (1993).

[38] V. Barger, M. S. Berger, and P. Ohmann, Madison Report No. MAD/PH/801 (1993).

[39] Similar issues were also studied by B. Ananthanarayan, K. S. Babu, and Q. Shafi, private communications. [After completion of this work we received their paper B. Ananthanarayan et al., Bartol Report No. BA-94-03 (1994).]

[40] We thank J. Erler for focusing our attention on that point.

[41] T. Kon and T. Nonaka, Seikei Report No. ITP-SU-93/05 (1993), and references therein.

[42] J. F. Gunion and H. Pois, UC Davis Report No. UCD-94-01 (1994). 
[43] M. Drees, M. Glück, and K. Grassie, Phys. Lett. 157B, 164 (1985).

[44] J. L. Lopez and D. V. Nanopoulos, Phys. Lett. B266, 397 (1991).

[45] J. Ellis, G. Ridolfi, and F. Zwirner, Phys. Lett B257, 83 (1991); ibid. 262, 477 (1991).

[46] A. Brignole, Phys. Lett. B281, 284 (1992).

[47] H. E. Haber and R. Hempfling, Phys. Rev. D 48, 4280 (1993).

[48] R. Hempfling, DESY Report No. DESY-93-012 (1993).

[49] G. Gamberini, G. Ridolfi, and F. Zwirner, Nucl. Phys. B331, 331 (1990).

[50] M. Claudson, L. J. Hall, and I. Hinchliffe, Nucl. Phys. B228, 501 (1983);

[51] A. Stange, W. Marciano, and S. Willenbrock, Phys. Rev. D 49, 1354 (1994).

[52] G. F. Giudice and E. Roulet, Phys. Lett. B315, 107 (1993), and references therein.

[53] J. F. Gunion, H. E. Haber, and M. Sher, Nucl. Phys. B306, 1 (1988).

[54] J. M. Frere, D. R. T. Jones, and S. Raby, Nucl. Phys. B222, 11 (1983); L. AlvarezGaume, J. Polchinski, and M. B. Wise, ibid. 221, 495 (1983); C. Kounnas, A. B. Lahanas, D. V. Nanopoulos, and M. Quiros, ibid. 236, 438 (1984); J. P. Derendinger and C. A. Savoy, ibid. 237, 307 (1984).

[55] H. Komatsu, Phys. Lett. B215, 323 (1988).

[56] S. Kelley, J. L. Lopez, D. V. Nanopoulos, H. Pois, and K. Yuan, Nucl. Phys. B398, 3 (1993).

[57] P. H. Chankowski, Phys. Rev. D 41, 2877 (1990).

[58] J. Kodaira, Y. Yasui, and K. Sasaki, Hiroshima Report No. HUPD-9316 (1993).

[59] R. Hempfling and A. H. Hoang, DESY Report No. DESY-93-162 (1993).

[60] B. Kastening, Phys. Lett. B283, 287 (1992); C. Ford, D. R. T. Jones, and P. W. Stephenson, Nucl. Phys. B395, 17 (1993).

[61] M. Bando, T. Kugo, N. Maekawa, and H. Nakano, Phys. Lett. B301, 83 (1993).

[62] J. R. Espinosa and M. Quiros, Phys. Lett. B266, 389 (1991).

[63] L. Durand and J. Lopez, Phys. Lett. B217, 463 (1989); M. Drees, Int. J. Mod. Phys. A 4, 3635 (1989).

[64] T. Elliott, S. F. King, and P. L. White, Southampton Report No. SHEP-92-93-21 (1993); and references therein.

[65] After completion of this work we received a paper by B. C. Allanach and S. F. King, Southampton Report No. SHEP-93-94-15 (1994), where similar issues are discussed. 


\section{FIGURES}

FIG. 1. The $t$-quark pole mass (in $\mathrm{GeV}$ ) - $\tan \beta$ plane is divided into five different regions. Two areas (low- and high-tan $\beta$ branches) are consistent with perturbative two-Yukawa ( $b$ and $\tau$ ) unification and with an upper bound of $\frac{4.45}{0.85} \mathrm{GeV}$ for the $b$-quark current mass. The 0.85 factor takes into account $\mathrm{a} \sim \pm 15 \%$ theoretical uncertainty in the numerical calculation due to the matching conditions. Between the two branches the $b$-quark mass is too high. For a too low (high) $\tan \beta$, the $t$-quark ( $b$-quark) Yukawa coupling diverges. The region where all three (third-family) Yukawa couplings unify (dashed line) intersects the allowed high- $\tan \beta$ branch. [The three-Yukawa region is calculated without imposing any constraints on $m_{b}$, and is assigned a $\sim 5 \%$ uncertainty (shaded area) from corrections to the $h_{t} / h_{b}$ ratio.] The $t$-quark mass range suggested by the electroweak data and the $\tan \beta=1$ line are indicated (dotted lines) for comparison.

FIG. 2. The prediction for the Higgsino mass parameter $\mu$ (after absorbing $\Delta V$ in the redefined tree-level parameters) as a function of $(a)$ the $t$-quark pole mass and of $(b) \tan \beta$. (The $t$-quark mass and $\tan \beta$ are strongly correlated.) Filled squares indicate (probably unacceptable) points for which the SM minimum is only a local minimum. All masses are in $\mathrm{GeV}$

FIG. 3. A scatter plot of the Higgs boson mass (calculated in the EPM with the subtraction scale $Q$ at the $Z$-pole) vs. the $t$-quark pole mass. The $\circ$ indicate points which have no (potentially dangerous) negative-energy color and/or charge breaking minimum. The $\diamond$ have such a minimum but it is a (safe) local one, i.e., it lies above the standard-model minimum. Points indicated by a filled diamond have an unacceptable CCB global minimum. The $Z$ mass is indicated for comparison (dashed line). All masses are in $\mathrm{GeV}$.

FIG. 4. Same as in Fig. 3 except the mass is calculated using the RGM (numerically).

FIG. 5. The EPM (with the subtraction scale $Q$ at the $Z$-pole) calculation of the Higgs boson mass is compared with the mass calculated in the RGM but using the Haber and Hempfling leading logarithm formula. All masses are in GeV. Points corresponding to global GCCB minima (indicated by a filled diamond in Figs. 3 and 4) are omitted.

FIG. 6. Same as in Fig. 5, except $Q=600 \mathrm{GeV}$ and the RGM calculation is carried out numerically.

FIG. 7. The Higgs boson mass (in $\mathrm{GeV}$ ) distribution in a sample monte-carlo calculation (using the EPM with the subtraction scale $Q$ at the $Z$-pole) for the $t$-quark pole mass in the range (a) $[155,165],(b)[165,175],(c)[175,185] \mathrm{GeV}$. 
FIG. 8. Same as in Fig. 7 except for the omission of points which correspond to a global color and/or charge breaking minimum (marked by a filled diamond in Fig. 3). For comparison, the shaded areas indicate points which are consistent with the constraint (13) (marked by a circle in Fig. 3). The difference between the two distributions is points with only a local CCB minimum unnecessarily excluded by (13) (marked by open diamonds in Fig. 3).

FIG. 9. Same as Fig. 7 except for substituting the $t$-quark running (rather than pole) mass in the mass expressions for the scalars.

FIG. 10. Same as in Fig. 7a-b except using the RGM (with numerical integration). The distributions omitting points which correspond to a global color and/or charge breaking minimum (marked by a filled diamond in Fig. 4) (lightly shaded areas) are also indicated, and should be compared with the total (shaded and unshaded) areas in Fig. 8. (For a $t$-quark heavier than about $180 \mathrm{GeV}$ the $1 \mathrm{HDM}$ assumption that underlies the calculation is not always accurate - see Appendix C.)

FIG. 11. The low $\tan \beta$ branch in the presence of an additional Higgs field with $\lambda_{s}=0.5$ and $\kappa_{s}=0$. The allowed area when there is no singlet field $\left(\lambda_{s}=0\right)$ is shown for comparison (shaded region). the $t$-quark pole mass (given in $\mathrm{GeV}$ ) range suggested by precision data and $\tan \beta=1$ are indicated (dotted lines). 


\section{TABLES}

TABLE I. The allowed regions in the $\tan \beta-m_{t}^{\text {pole }}$ plane. Relations (1) and (2) are also consistent with the parameter space described by case (2) except that $h_{t} \geq h_{b} . m_{\tilde{q}}$ stands for a typical scalar-quark mass. Finite corrections $\propto \tan \beta M_{\frac{1}{2}} \mu / m_{0}^{2}$ could modify the allowed $m_{t}^{\text {pole }}$ range in case (2).

\begin{tabular}{ccc}
\hline \hline & case $(1)$ & case $(2)$ \\
\hline \hline GUT relations & $(1)$ and $(2)$ & $(1)$ and (3) \\
The allowed $m_{t}^{\text {pole }}$ range $(\mathrm{GeV})$ & $140-\sim 190$ & $\sim 180 \pm 15$ \\
The allowed tan $\beta$ range & $1-2$ & $50 \pm 10$ \\
Yukawa couplings & $h_{t} \approx 1 \gg h_{b}$ & $h_{t} \approx h_{b} \approx 1$ \\
The Higgs potential (approximate) symmetry & $S U(2)_{L} \times S U(2)_{R}$ & $O_{4} \times O_{4}$ \\
The vacuum (approximate) symmetry & $S U(2)_{L+R}$ & $O_{3} \times O_{3}$ \\
$S U(2) \times U(1)$ breaking "messenger" & $m_{3}^{2}$ & $m_{H_{2}}^{2}$ \\
The large mass parameter & Higgsino mass parameter & scalar-quark masses \\
$m_{h^{0}}^{T}$ & $m_{h^{0}}^{T} \approx 0$ & $m_{h^{0}}^{T} \approx M_{Z}$ \\
$\Delta_{h^{0}}$ enhancement & left-right mixings & large $m_{t}$ and the heavy scalars \\
$m_{H^{0}}$ & $m_{H^{0}} \approx m_{A^{0}}$ & $m_{H^{0}} \approx m_{A^{0}}$ \\
$A^{0}, H^{+}$ & heavy & massive pseudo-Goldstone bosons \\
& $m_{A^{0}} \approx \sqrt{2}|\mu| \approx 2$ TeV & $m_{A^{0}} \ll m_{\tilde{q}}$ \\
\hline \hline
\end{tabular}




\title{
Implications of Yukawa unification for the Higgs sector in supersymmetric grand-unified models
}

\author{
Paul Langacker and Nir Polonsky \\ Department of Physics, University of Pennsylvania, Philadelphia, Pennsylvania, 19104, USA
}

(February 1994, UPR-0594T)

\begin{abstract}
The $S U(5)$ unification-scale relation $h_{b}=h_{\tau}$ between the $b$-quark and $\tau$ lepton Yukawa couplings severely constrains $\tan \beta$ and the $t$-quark mass (even more so if $h_{t}=h_{b}=h_{\tau}$ holds) in supersymmetric models. We examine the implications of these constraints for the Higgs sector assuming universal soft breaking terms, and emphasize that both of these relations impose unique characteristics in terms of symmetries and of the spectrum. We further study the $\tan \beta \approx 1$ scenario, which is suggested by $h_{b}=h_{\tau}$, and, in particular, the loop-induced mass of the light Higgs boson. We compare the effective potential and renormalization group methods and stress the two-loop ambiguities in the calculation of the mass. These and a large enhancement to the loop correction due to $t$-scalar left-right mixing considerably weaken the upper bound on the mass of the light Higgs boson that has been reported. Nevertheless, we find that for this scenario the Higgs boson is probably lighter than $110 \mathrm{GeV}$, and typically lighter than $100 \mathrm{GeV}$. Thus, it is in the mass range that may be relevant for LEPII. Our numerical results are presented in a self-contained manner in section $V$. In separate appendices we discuss the global symmetries of the Higgs potential, the issue of false (color-breaking) vacua, which may be important for $\tan \beta \approx 1$, two-loop calculations, and the effect of an additional Higgs singlet. We show that the approximate constraints that are often used to eliminate color-breaking vacua are not always relevant.
\end{abstract}

PACS numbers: 12.10.Dm, 11.30.Pb, 14.80.Gt, 14.80.Ly 


\section{INTRODUCTION}

Supersymmetric grand-unified theories [1,2] (SGUT), though far from being proved, are an attractive framework on both observational and theoretical grounds. (Their relation to string theory remains at present poorly understood.) In the minimal models, which we assume hereafter, there is a grand desert between the weak and the unification scales ( $M_{Z} \lesssim Q \lesssim \mathrm{TeV}$ and $M_{G} \approx 10^{16}-10^{17} \mathrm{GeV}$, respectively). At the unification point $M_{G}$

$$
\alpha_{1}\left(M_{G}\right)=\alpha_{2}\left(M_{G}\right)=\alpha_{3}\left(M_{G}\right) \equiv \alpha_{G}
$$

(where $\alpha_{1}=\frac{5}{3} \alpha_{Y}$ and $\alpha_{G} \approx 0.04$ ), and, depending on the grand unifying group representations of the Higgs superfields that couple to matter, certain relations between the standard model Yukawa couplings hold. In the minimal models based on $S U(5), S O(10)$, and $E_{6}$ these representations are the fundamental ones, and one obtains [3]

$$
h_{\tau}\left(M_{G}\right)=h_{b}\left(M_{G}\right) .
$$

For some models based on $S O(10)$ (or larger groups) one has the stronger prediction

$$
h_{\tau}\left(M_{G}\right)=h_{b}\left(M_{G}\right)=h_{t}\left(M_{G}\right)
$$

but this only holds if one makes the additional assumption that the masses are generated by a single complex Higgs 10-plet. ( $h_{\tau, b, t}$ are the SM Yukawa couplings of the $\tau, b, t$-superfields, respectively.) One usually assumes that some perturbation modifies the coupling or the masses of the two light families where, in principle, similar relations should, but do not, hold ${ }^{1}$. (See also Appendix D.) Also, such relations often do not hold in superstring unified models.

At the weak-scale the particle content is that of the minimal supersymmetric standard model (MSSM), i.e., that of a two Higgs doublet model but with each bosonic (fermionic) degree of freedom complemented by a fermionic (bosonic) one. In particular, the Higgs sector contains three Goldstone bosons and five physical degrees of freedom - two CP even $\left(h^{0}\right.$ and $\left.H^{0}\right)$ and one $\mathrm{CP}$ odd $\left(A^{0}\right)$ neutral, and one complex charged $\left(H^{+}\right)$Higgs bosons. The symmetries and spectrum of the Higgs scalars are strongly affected by the above GUT assumptions, and are the subject of our present study. However, a review of previous results in SGUT's, which establish the base for our present work, is appropriate.

The consistency of (1) with the data was pointed out recently in Ref. [4] and that of (2) and (3) in Ref. [5]. Relation (1), however, holds only if there is exactly one pair of Higgs doublets (but any number of SM singlets is allowed). We previously investigated the status of coupling constant unification in great detail [6] where we considered non-trivial matching conditions ${ }^{2}$ that perturb relation (1) in a model dependent way. We concluded

\footnotetext{
${ }^{1}$ Since the Yukawa couplings corresponding to the third family are much larger than those of the two light families, relation (2) [or (3)] will be only slightly affected.

${ }^{2}$ Heavy degrees of freedom (which are model dependent) are integrated out of the effective theory
} 
that no significant constraints on the MSSM parameters can be deduced just by that relation. However, we find ${ }^{3}$ that (1) predicts for the strong coupling [using $\alpha\left(M_{Z}\right)^{-1}=127.9 \pm 0.1$ [7] and $s^{2}\left(M_{Z}\right)=0.2324 \pm 0.0003$ (for $m_{t}^{\text {pole }}=143 \mathrm{GeV}$ ) for the $(\overline{\mathrm{MS}}$ ) fine-structure constant and weak angle, respectively]

$$
\alpha_{s}\left(M_{Z}\right)=0.125 \pm 0.001 \pm 0.008+H_{\alpha_{s}}+3.2 \times 10^{-7} \mathrm{GeV}^{-2}\left[\left(m_{t}^{\text {pole }}\right)^{2}-(143 \mathrm{GeV})^{2}\right]
$$

where the $\pm 0.008( \pm 0.001)$ uncertainty in (4) is theoretical (due to the input parameter error bars). The former is due to the unknown values of the matching conditions (i.e., threshold and nonrenormalizable-operator effects) mentioned above. The function $H_{\alpha_{s}}$ is a correction from Yukawa interactions. It is negative but negligible, unless some Yukawa couplings are $\gtrsim 1$, for which we obtain $H_{\alpha_{s}} \approx-(0.001-0.003)$. The quadratic $m_{t}^{\text {pole }}$ dependence is induced by the correlation between the weak angle extracted from the data and the $t$-quark pole mass, $m_{t}^{\text {pole }}$. Thus, typically one predicts $\alpha_{s}\left(M_{Z}\right) \gtrsim 0.12$ with a significant dependence on $m_{t}^{\text {pole }}$. This observation is of importance when simultaneously considering (1) and (2) [or (3)] to predict the $b$-quark ( $\overline{\mathrm{MS}}$ running) mass $m_{b}$ [and/or the $t$-quark mass, if using (3)] in terms of the $\tau$-lepton mass. We find [using, e.g., Eq. (4) but with a more careful treatment of theoretical uncertainties to the $b$-quark mass prediction] that only a small region in the $\tan \beta-m_{t}^{\text {pole }}$ plane is allowed by the experimental range of $m_{b}[8]$, where $\tan \beta=\nu_{\text {up }} / \nu_{\text {down }}$ is the ratio of the two Higgs doublet expectation values. This holds even when non-trivial matching conditions at both scales are considered.

It is customary to assume that the MSSM spectrum is given to a good approximation near $M_{G}$ by a small number of universal supersymmetry-breaking soft parameters; i.e., a common scalar mass $m_{0}$, trilinear and bilinear (dimension-one) couplings $A_{0}$ and $B_{0}$, and a common gaugino mass $M_{\frac{1}{2}}$; in addition to a supersymmetric Higgs mass (i.e., the Higgsino mass), $\mu_{0}$ [2]. We will adopt these assumptions below. (For an alternative scenario, see, for example, Ref. [9].) However, our conclusions so far are largely (i.e., except for the the details of the matching conditions) independent of those assumptions and hold in any SGUT scenario with an approximate grand-desert in which the MSSM $\beta$-functions are valid. The universality assumption just strengthens the constraints on the $\tan \beta-m_{t}^{\text {pole }}$ plane; i.e., assuming universal initial conditions and requiring that the weak-scale MSSM (one-loop improved) scalar potential is consistent with a broken $S U(2) \times U(1)$ symmetry, we have to further constrain $\tan \beta \geq 1$ and $h_{t} \geq h_{b}$. We then find two allowed regions,

1. $\tan \beta=1+\Delta_{\beta}, 140 \lesssim m_{t}^{\text {pole }}(\lesssim 200) \mathrm{GeV}, h_{t} \approx 1 \gg h_{b}$;

2. $\tan \beta \approx 50 \pm 10,170 \pm 10 \lesssim m_{t}^{\text {pole }}(\lesssim 200) \mathrm{GeV}, h_{t} \gtrsim h_{b} \approx 1$.

at $M_{G}$ and some modification to the naive boundary conditions in (1) and (2) [or (3)] is required, in general. The appropriate matching functions may also include corrections from nonrenormalizable operators since $M_{G}$ is not too far from the Planck scale. Similarly, integrating out the MSSM new particle spectrum will correct the boundary conditions at $M_{Z}$.

${ }^{3} \mathrm{Eq}$. (4) updates the results in [6] for the effect of more recent precision electroweak data. 
The first of these solutions is consistent only with $h_{b}=h_{\tau}$, and has $\Delta_{\beta} \lesssim 1$ for $m_{t}^{\text {pole }} \lesssim 190$ $\mathrm{GeV}$. A part of the region which is described by the second solution is consistent with the stronger prediction $h_{\tau}=h_{b}=h_{t}$. A third solution with $3 \lesssim \tan \beta \lesssim 40$ exists for $m_{t}^{\text {pole }} \approx 215 \pm 10 \mathrm{GeV}$, which is the upper bound on $m_{t}^{\text {pole }}$ if the model is to stay perturbative up to $M_{G}$. However, such large values for $m_{t}$ are inconsistent with precision electroweak data. The allowed regions are illustrated in Fig. 1.

These results are in good agreement with those of Ref. [10-14], with any small differences due to different treatments of matching conditions and of the coupling constant unification condition. Arbitrarily relaxing both condition (1) and (2) independently [e.g., $\alpha_{s}\left(M_{Z}\right)=$ 0.11 and $\left.h_{b}\left(M_{G}\right)=0.85 h_{\tau}\left(M_{G}\right)\right]$ could invalidate our conclusions. However, the corrections to (1) and (2) are strongly correlated, and when treated as such the strong constraints on the $\tan \beta-m_{t}^{\text {pole }}$ plane hold ${ }^{4}$. In particular, the $m_{b} / m_{\tau}$ ratio is relatively insensitive to the details of the decoupling of the low-scale spectrum [8], and our conclusions would not be significantly modified if those thresholds were treated differently than in our (semianalytic) approximation (where we used six effective mass parameters). Let us stress that any treatment of theoretical uncertainties would be lacking unless the potentially important corrections at the high scale are accounted for in one form or another. These issues are summarized in Ref. [15,16], where the second reference updates some of the results of Ref. $[6,8]$. Here, relevant results are again updated using a preliminary analysis of most recent precision data.

A two-parameter fit [allowing the light (SM-like) Higgs boson mass, $m_{h^{0}}$, to vary from $50-150 \mathrm{GeV}$ with a central value $m_{h^{0}}=M_{Z}$ ] to all $Z, W$, and neutral-current data yields

$$
\begin{gathered}
s^{2}\left(M_{Z}\right)=0.2324 \pm 0.0003-0.92 \times 10^{-7} \mathrm{GeV}^{-2}\left[\left(m_{t}^{\text {pole }}\right)^{2}-(143 \mathrm{GeV})^{2}\right] \\
m_{t}^{\text {pole }}=143_{-19}^{+17}+12.5 \ln \frac{m_{h^{0}}}{M_{Z}} \mathrm{GeV}
\end{gathered}
$$

which in turn implies $\tan \beta \lesssim 1.3$ (at one s.d.). Eqs. (5) and (6) update Ref. [17] where $m_{t}^{\text {pole }}=134_{-28}^{+23}+12.5 \ln \frac{m_{h 0}}{M_{Z}} \mathrm{GeV}$ was given (which also implied $\tan \beta \lesssim 1.3$ ).

It was pointed out by Barger et al. [12] that if indeed $m_{t}^{\text {pole }} \lesssim 160 \mathrm{GeV}$ (as suggested by the range given in Ref. [17] and here) then $m_{h^{0}}$ [in case (1)] is determined by the magnitude of the loop corrections, $\Delta_{h^{0}}$, and is $\lesssim 85 \mathrm{GeV}$. If so, the $b$-quark mass prediction in minimal $S U(5)$ (or similar) SGUT constrains the SM Higgs boson mass more significantly than the general MSSM (triviality) upper bound of $\sim 130 \mathrm{GeV}$ [18-20]. We agree qualitatively with this conclusion, but argue that two-loop ambiguities and important left-right $t$-scalar mixing effects, in addition to the higher $m_{t}^{\text {pole }}$ range, weaken the bound to $m_{h^{0}} \lesssim 110 \mathrm{GeV}$.

Below, we will establish the basis for and then pursue further numerical studies of the prediction for $m_{h^{0}}$ and its upper bound in the $\tan \beta \approx 1$ scenario. Rather than approximating the one-loop correction we will calculate $m_{h^{0}}$ for a given point in the parameter space using the supersymmetric spectrum (calculated numerically), and use monte-carlo routines to study the upper bound. In section II we briefly review the MSSM Higgs potential and its

\footnotetext{
${ }^{4}$ That is unless one assumes a conspiracy of various corrections that are otherwise independent.
} 
minimization conditions. We specialize the discussion to the minimal SGUT scenarios, cases (1) and (2), and emphasize that the Higgs potential exhibits approximate global symmetries in these cases, on which we elaborate in Appendix A. We concentrate thereafter on the $\tan \beta \approx 1$ scenario. Some general features are described in section III. In particular, the large Higgsino mass parameter can generate false vacuum solutions with broken color and charge. We discuss that issue in greater detail in Appendix B. The calculation of the light Higgs boson mass in both the effective potential and renormalization group methods is discussed in section IV, where we use the comparison between the two methods to study the two-loop ambiguities in the calculation. The details of the two methods, as well as two-loop calculations, are further discussed in Appendix C. Section V is reserved for further numerical studies of the Higgs boson mass, and we show that because of two-loop ambiguities and $\tilde{t}_{L}-\tilde{t}_{R}$ mixing $m_{h^{0}}$ can easily exceed $85 \mathrm{GeV}$ but, unless $m_{t}^{\text {pole }}$ is much heavier than the range suggested by (6), it remains in the range that may be relevant for LEPII. There is even some possibility that it is in the range still relevant for LEPI. We also discuss the relation between $t$-scalar mixing and color breaking. In particular, parameter ranges which correspond to large mixing (and large $m_{h^{0}}$ ) often have an unacceptable color-breaking global minimum, but not always. The discussion in section $\mathrm{V}$ is to a great extent independent of the other sections and can be read on its own. We will summarize our conclusions in section VI, where we also examine the implications for $m_{t}^{\text {pole }}$. In Appendix D we point out simple extensions of either the MSSM or the GUT in which our (constrained) analysis does not apply.

Below, we point out that (negative-energy) color-breaking local minima of the full scalar potential are generic. Approximate analytic constraints that are often used in the literature attempt to eliminate not only global but also local color-breaking minima, and are thus too strong. Furthermore, they are designed to eliminate only certain types of color-breaking minima, and are thus too weak. This is explicitly demonstrated in Appendix B, where we also compare analytic and numerical treatments of the problem.

We follow Ref. [8] in calculating the couplings (and the unification point), and Ref. [21] in treating the one-loop effective potential correction $\Delta V$, including contributions from all sectors. The boundary conditions at $M_{G}$ for $0 \leq m_{0} \leq 500 \mathrm{GeV},\left|A_{0}\right| \leq 3 m_{0}$, and $50 \leq M_{\frac{1}{2}} \leq 350 \mathrm{GeV}$, are picked at random. $m_{t}^{\text {pole }}$ values are picked at random but with a gaussian distribution defined by $(6)$. For a given $m_{t}^{\text {pole }}, \tan \beta\left(M_{Z}\right)$ is picked at random within its allowed region [case (1)]. Values of $m_{t}^{\text {pole }}<155 \mathrm{GeV}$ are discarded so that $\tan \beta \gtrsim 1.1$ and the divergent limit is not reached (see below). We then solve iteratively for $\mu_{0}^{2}$ (and $B_{0}$ ). [The relevant renormalization group equations (RGE's) have been given by various authors [22].] The sign of $\mu_{0}$, which is a RG invariant, is picked at random as well. We explicitly verify that the potential is bounded from below; $S U(2) \times U(1)$ is properly broken; the lightest supersymmetric particle (LSP) is neutral (in practice, it is a neutralino); and that all the squared masses which correspond to physical scalars are positive before a point is accepted. We then find, in general, that we are in agreement with lower bounds coming from direct searches. (e.g., values of $M_{\frac{1}{2}} \lesssim 100 \mathrm{GeV}$, which would imply a too light chargino, are already excluded in our case by a tachionic $t$-scalar.) We do not impose a lower bound on $m_{h^{\circ}}$, which is the subject of our investigation. Our treatment of color and/or charge breaking (CCB) minima is discussed in Appendix B. 


\section{THE WEAK-SCALE HIGGS SECTOR}

The Higgs part of the MSSM (weak-scale) scalar potential reads [23,18]

$$
\begin{gathered}
V\left(H_{1}, H_{2}\right)=\left(m_{H_{1}}^{2}+\mu^{2}\right)\left|H_{1}\right|^{2}+\left(m_{H_{2}}^{2}+\mu^{2}\right)\left|H_{2}\right|^{2} \\
+B \mu\left(H_{1} H_{2}+\text { h.c. }\right)+\frac{\lambda^{\mathrm{MSSM}}}{2}\left(\left|H_{2}\right|^{2}-\left|H_{1}\right|^{2}\right)^{2}+\Delta V,
\end{gathered}
$$

where $m_{H_{1}}^{2}, m_{H_{2}}^{2}$, and $B(\mu)$ are the soft (supersymmetric) mass parameters renormalized down to the weak scale, $m_{3}^{2} \equiv B \mu<0, \lambda^{\mathrm{MSSM}}=\frac{g_{2}^{2}+\frac{2}{5} g_{1}^{2}}{4}$, and we suppress $S U(2)$ indices. The one-loop correction $[24,18] \Delta V=\Delta V^{\text {one-loop }}$ (which, in fact, is a threshold correction to the one-loop improved tree-level potential) can be absorbed to a good approximation in redefinitions of the tree-level parameters [21,25].

A broken $S U(2) \times U(1)$ (along with the constraint $m_{H_{1}}^{2}+m_{H_{2}}^{2}+2 \mu^{2} \geq 2\left|m_{3}^{2}\right|$ from vacuum stability) requires $[23,18]$

$$
\left(m_{H_{1}}^{2}+\mu^{2}\right)\left(m_{H_{2}}^{2}+\mu^{2}\right) \leq\left|m_{3}^{2}\right|^{2},
$$

and the minimization conditions then give $[23,18]$

$$
\begin{gathered}
\mu^{2}=\frac{m_{H_{1}}^{2}-m_{H_{2}}^{2} \tan ^{2} \beta}{\tan ^{2} \beta-1}-\frac{1}{2} M_{Z}^{2}, \\
m_{3}^{2}=-\frac{1}{2} \sin 2 \beta\left[m_{H_{1}}^{2}+m_{H_{2}}^{2}+2 \mu^{2}\right] .
\end{gathered}
$$

For $\tan \beta \rightarrow 1$ one has $h_{t} \gg h_{b}$, and hence cannot have $m_{H_{1}}^{2}=m_{H_{2}}^{2}$ (assuming universal initial conditions). Thus, $|\mu| \rightarrow \infty$ in that limit [case (1)], and the $S U(2) \times U(1)$ breaking is driven by the $B \mu$ term. In practice, we will stay away from the divergent limit in case (1) by taking $\tan \beta \gtrsim 1.1$ [21]. We do not expect significantly different results for $1 \leq \tan \beta \leq 1.1$; i.e., the divergence is either stabilized by model dependent finite-loop corrections or would exclude that region (see also below). For $\tan \beta \rightarrow \infty$ [case (2)] one has $B \mu \rightarrow 0$ so that the symmetry breaking is driven by $m_{H_{2}}^{2}<0$.

The $\tan \beta \rightarrow 1$ case corresponds to an approximate $S U(2)_{L+R}$ custodial symmetry of the vacuum $[26,27]$, which we further discuss in Appendix A. The symmetry is broken at the loop level so that one expects $\tan \beta$ slightly above unity, in agreement with our cut $\tan \beta \gtrsim 1.1$. As a result of the symmetry, the CP-even Higgs mass matrix becomes

$$
M^{2} \approx \mu^{2} \times\left(\begin{array}{cc}
1 & -1 \\
-1 & 1
\end{array}\right),
$$

and it has a massless tree-level eigenvalue, $m_{h^{0}}^{T} \approx 0$. This is, of course, a well known result of the tree-level formula [23,18]

$$
m_{h^{0}}^{T}{ }^{2}=\frac{1}{2}\left[m_{A^{0}}^{2}+M_{Z}^{2}-\sqrt{\left(m_{A^{0}}^{2}+M_{Z}^{2}\right)^{2}-4 m_{A^{0}}^{2} M_{Z}^{2} \cos ^{2} 2 \beta}\right]
$$


when taking $\beta=\frac{\pi}{4}$. The mass is then determined by the loop correction $m_{h^{0}} \approx \Delta_{h^{0}} \propto h_{t} m_{t}$ and is further studied in sections IV and V. On the other hand, in case (2) $\beta \rightarrow \frac{\pi}{2}$ and we have $m_{h^{0}}^{T} \approx M_{Z}$ (assuming $m_{A^{0}} \geq M_{Z}$ ). When adding the loop correction ${ }^{5} m_{h^{0}} \lesssim \sqrt{2} M_{Z} \approx 130$ $\mathrm{GeV}$. The heavier CP-even Higgs boson mass eigenvalue equals approximately $\sqrt{2}|\mu|$ in case (1) and $m_{H^{0}} \approx m_{A^{0}}$ in case $(2)$. The loop corrections are less relevant here as typically $m_{H^{\circ}}^{2} \gg \Delta_{H^{0}}^{2}$ (in particular, when $\left.|\mu| \rightarrow \infty\right)$.

The custodial symmetry (or the large $\mu$ parameter) dictates in case (1) a degeneracy $m_{A^{0}} \approx m_{H^{0}} \approx m_{H^{+}} \approx \sqrt{2}|\mu|$. [The tree-level corrections to that relation are of order $\left(M_{W, Z} / m_{A^{0}}\right)^{2}$.] That is, at a scale $Q \approx \sqrt{2}|\mu| \approx 2 \mathrm{TeV}$ the heavy Higgs doublet $H$ is decoupled, and the effective field theory below that scale has only one massless $\left(m_{h^{0}}^{T} \approx 0\right)$ Coleman-Weinberg SM-like $\left(\nu_{h^{0}}=\nu\right)$ Higgs doublet, $h$. Unlike in our case, in general $M_{Z} / m_{A^{0}}$ is not guaranteed to be small, and the decoupling scale $Q$ is much lower, typically below the scalar-quark thresholds.

The Higgs sector in case (2) exhibits an approximate $O_{4} \times O_{4}$ symmetry (see Appendix A). This is a special case of the $m_{3}^{2} \rightarrow 0$ case discussed in Ref. [28]. To obtain $m_{3}^{2} \approx 0$ (starting with universal initial conditions and $h_{b} \approx h_{t}$ ) one would require large $M_{\frac{1}{2}}$ and $\left|\mu_{0}\right|$, which enable one to obtain the desired limit by adjusting large cancellations [29-31]. Once expectation values are acquired, $A^{0}$ and $H^{+}$are massive pseudo-Goldstone bosons of the broken $O_{4}$ symmetry. However, they are typically heavy (for $\nu_{\text {down }}=0$ the $O_{4}$ symmetry is restored) but lighter than the scalar quarks in this scenario (whose mass $m_{\tilde{q}} \approx$ $\left.\sqrt{m_{0}^{2}+6 M_{\frac{1}{2}}^{2}} \approx 1 \mathrm{TeV}\right)$

Large $\tan \beta$ [case (2)] solutions are discussed in Ref. [29,30,13,32,31]. They predict $m_{t}^{\text {pole }} \approx 180 \pm 15 \mathrm{GeV}[8,13]$, heavy scalars, and contain potentially troublesome loop corrections, e.g., to $m_{b}, b \rightarrow s \gamma[13,32]$; and, in the absence of light scalars to counterbalance large $m_{t}$ contributions, also to $Z \rightarrow b \bar{b}$ (see Ref. [33]). The former can eliminate the motivation for any Yukawa coupling analysis. A different approach was recently presented in Ref. [31] where those large finite-loop corrections were used to construct models consistent with universality, a broken $S U(2) \times U(1)$, and $h_{t}=h_{b}=h_{\tau}$. Finite superpartner $(\sim 1 \mathrm{TeV})$ loops are adjusted to diminish $m_{b}$ by the right amount $(\sim 1 \mathrm{GeV})$, and $m_{b}$ is strongly dependent on the values of the soft parameters, a possible but undesirable situation. Much lower values of $m_{t}^{\text {pole }}$ are then preferred. Diminishing $m_{t}$ may rectify the situation with $Z \rightarrow b \bar{b}$, but the prediction for the $b \rightarrow s \gamma$ branching ratio can be significantly modified by the same superpartner loops [13]. In contrast, the $\tan \beta \approx 1$ [case (1)] solution constrains only $m_{t}^{\text {pole }} \gtrsim 140$ $\mathrm{GeV}\left(m_{t}^{\text {pole }} \gtrsim 155 \mathrm{GeV}\right.$ when imposing the $\tan \beta \gtrsim 1.1$ cut $)$, is in better agreement with (6), and predicts a light Higgs boson $\left(m_{h^{0}} \lesssim 110 \mathrm{GeV}\right)$. Cases $(1)$ and $(2)$ are summarized and compared in Table I. We hereafter study the $\tan \beta \approx 1$ [case (1)] solution only. We assume that finite-loop corrections to $m_{b}$ are at the most a few percent which is, in general, the case away from the large $\tan \beta$ limit, and thus do not alter our results. [The parameter space is sensitive to those corrections only in case (2).]

\footnotetext{
${ }^{5} \Delta_{h^{0}}$ is enhanced in this case by the large $m_{t}$ and the heavy $t$-scalars.
} 
TABLE I. The allowed regions in the $\tan \beta-m_{t}^{\text {pole }}$ plane. Relations (1) and (2) are also consistent with the parameter space described by case (2) except that $h_{t} \geq h_{b} . m_{\tilde{q}}$ stands for a typical scalar-quark mass. Finite corrections $\propto \tan \beta M_{\frac{1}{2}} \mu / m_{0}^{2}$ could modify the allowed $m_{t}^{\text {pole }}$ range in case (2).

\begin{tabular}{ccc}
\hline \hline & case $(1)$ & case $(2)$ \\
\hline \hline GUT relations & $(1)$ and $(2)$ & $(1)$ and $(3)$ \\
The allowed $m_{t}^{\text {pole }}$ range $(\mathrm{GeV})$ & $140-\sim 190$ & $\sim 180 \pm 15$ \\
The allowed tan $\beta$ range & $1-2$ & $50 \pm 10$ \\
Yukawa couplings & $h_{t} \approx 1 \gg h_{b}$ & $h_{t} \approx h_{b} \approx 1$ \\
The Higgs potential (approximate) symmetry & $S U(2)_{L} \times S U(2)_{R}$ & $O_{4} \times O_{4}$ \\
The vacuum (approximate) symmetry & $S U(2)_{L+R}$ & $O_{3} \times O_{3}$ \\
$S U(2) \times U(1)$ breaking "messenger" & $m_{3}^{2}$ & $m_{H_{2}}^{2}$ \\
The large mass parameter & Higgsino mass parameter & scalar-quark masses \\
$m_{h^{0}}^{T}$ & $m_{h^{0}}^{T} \approx 0$ & $m_{h^{0}}^{T} \approx M_{Z}$ \\
$\Delta_{h^{0}}$ enhancement & left-right mixings & large $m_{t}$ and the heavy scalars \\
$m_{H^{0}}$ & $m_{H^{0}} \approx m_{A^{0}}$ & $m_{H^{0}} \approx m_{A^{0}}$ \\
$A^{0}, H^{+}$ & heavy & massive pseudo-Goldstone bosons \\
& $m_{A^{0}} \approx \sqrt{2}|\mu| \approx 2$ TeV & $m_{A^{0}} \ll m_{\tilde{q}}$
\end{tabular}

\section{THE $\tan \beta \rightarrow 1$ SCENARIO}

Our primary motivation to study the $\tan \beta \approx 1$ solution to the MSSM is its consistency with condition (2). The large values of $\alpha_{s}\left(M_{Z}\right)$ implied by (1) [e.g., Eq. (4)] generate (too) large positive loop corrections to $h_{b} / h_{\tau}$, which need to be counterbalanced by large negative corrections $\propto h_{t}^{2}, h_{b}^{2}$. The latter are sufficient only if some of the the Yukawa couplings are large, i.e., near their unitarity upper bounds, which is the case in the allowed regions. [Also, $H_{\alpha_{s}}$ in (4) becomes non-negligible in that limit.] The large values of the Yukawa couplings can be understood in terms of a quasi-fixed point in the flow of the respective RGE's [34]. In particular, in case (1) $h_{t}$ is near its quasi-fixed point. The two issues, i.e., the consistency of $\tan \beta \approx 1$ with $(2)$ and the $h_{t}$-RGE flow structure, are strongly related.

The smallness of $\Delta_{\beta}$ (or alternatively, the large $|\mu|$ ) can be understood in terms of an approximate $S U(2)_{L} \times S U(2)_{R}$ symmetry in the Higgs potential, which, if it exists at some scale, will be only slightly broken at $M_{Z}$, as is shown in Appendix A. The $\tan \beta \approx 1$ scenario was previously studied in Ref. [35], where it was referred to as "highest classical degeneracy": in the $\tan \beta=1$ limit condition (8) becomes an equality. [In practice, we find that $\left(m_{H_{1}}^{2}+\mu^{2}\right)\left(m_{H_{2}}^{2}+\mu^{2}\right) / m_{3}^{4} \gtrsim 0.99$ for $\Delta_{\beta}<1$ and after the proper redefinitions.] It was subsequently studied in Ref. [36] in the context of a global MSSM (i.e., no high-scale assumptions), and its consistency with limits on $m_{h^{0}}$ was shown. The $\tan \beta \approx 1$ solution suggested by the minimal SGUT relation $h_{b}=h_{\tau}$ revived the interest in that corner of parameter space. More recently Ref. [37-39], motivated by the quasi-fixed point prediction to $m_{t}$, extended the discussion from the Higgs sector to the full parameter space.

In Figs. $2 \mathrm{a}$ and $\mathrm{b}$ we show the prediction for $\mu$ as a function of $m_{t}^{\text {pole }}$ and of $\tan \beta$, respectively. Typically $|\mu| \sim 1 \mathrm{TeV}$, depending on $\Delta_{\beta}$ and on the soft parameter scale. It is 
the large $|\mu|$ parameter that dictates the characteristics of the scenario (and not only for the Higgs sector). Before further elaborating on the calculation of $m_{h^{0}}$, let us briefly summarize some of the features that appear in our numerical studies, and which are of relevance for the discussion below. Though we agree on the characteristics with other authors, our emphasis and interpretation are different.

1. The Higgsino (with mass $\sim \mu$ ) is decoupled from the (much lighter) gauginos. The bino and the wino are the lightest neutralino and chargino, respectively. (The former is the LSP which has a bino fraction near unity.) The heavy Higgsino decouples from the $Z \rightarrow b \bar{b}$ vertex, and thus, smaller values of $m_{t}^{\text {pole }}$ are favored ${ }^{6}$. However, $m_{t}^{\text {pole }}$ and $\tan \beta$ are correlated, and as $m_{t}^{\text {pole }}$ grows $|\mu|$ is diminished (see Fig. 2). Thus, in principle, Higgsino - $t$-scalar loops can still counterbalance large $m_{t}$ contributions to $Z \rightarrow b \bar{b}$ (see, for example, Ref. [33]) if the $t$-scalar is light enough. [A light $t$-scalar is, however, less likely if $|\mu|$ is small (see below) and from the $\rho$-parameter.]

2. The $t$-scalar mass-squared matrix is (we take all parameters to be real)

$$
\left(\begin{array}{cc}
m_{\tilde{t}_{L}}^{2} & m_{t}\left(A_{t}+\mu / \tan \beta\right) \\
m_{t}\left(A_{t}+\mu / \tan \beta\right) & m_{\tilde{t}_{R}}^{2}
\end{array}\right),
$$

and similarly for the superpartners of the other fermions. The diagonal elements correspond to the left and right-handed $t$-scalar squared masses, $m_{\tilde{t}_{L, R}}^{2}$, which consist of soft, $F$, and $D$ terms. $A_{t}$ is the trilinear soft parameter preceeding the $h_{t} H_{2} \tilde{t}_{L} \tilde{t}_{R}$ term in the scalar potential, with $A_{t}\left(M_{G}\right)=A_{0}$. The prediction for the diagonal terms has no unique charachteristics in our case, and the left and right-handed masses are in the $\sim 100 \mathrm{GeV}-1 \mathrm{TeV}$ range. However, the mixing term is typically large (unless the fermion mass is $\approx 0$ as is the case for the light families). In the limit $|\mu| \rightarrow \infty$ there is a $\theta_{t}=\frac{\pi}{4}$ left-right mixing so that one of the eigenstates would have a negative mass squared, and the limit cannot be fully realized. In practice, the soft terms and $m_{t}|\mu|$ are often of the same order of magnitude, with a nearly degenerate mass-squared matrix. Thus, the requirement that (9) defines a physical minimum with no negative squared masses, corresponding to physical scalars, constrains $\mu$. Also, our previous comment regarding a light $t$-scalar and a small $\mu$-parameter is clear by observation of (12). It is interesting to note that by fine adjustments of the soft parameters [i.e., of the degeneracy in (12)] one may realize a scenario with a light $(\lesssim 45 \mathrm{GeV}) t$-scalar which is nearly decoupled from the $Z$ (e.g., $\sin \theta_{t} \approx 0.8$ ). (See, for example, Ref. [41].) However, such scenarios are often associated with false vacua (see below and Appendix $\mathrm{B})$.

3. The absence of a negative $t$-scalar squared mass determines a ( $m_{0}$ and $\Delta_{\beta}$ dependent) lower bound on $M_{\frac{1}{2}}$. In particular, "no-scale" (i.e., $m_{0}=A_{0}=0$ ) SGUT models are

${ }^{6}$ The prediction for the $Z \rightarrow b \bar{b}$ branching ratio decreases with $m_{t}^{\text {pole }}$, and its measured value is $\sim 1.5$ s.d. higher than the SM prediction with $m_{t}^{\text {pole }} \approx 140 \mathrm{GeV}$. The discrepancy grows with $m_{t}^{\text {pole }}$ $[40]$. 
strongly constrained ${ }^{7} 8$. Also, requiring that the light eigenstate of the $\tau$-scalar masssquared matrix (which carries charge) is not the LSP determines an upper bound on $M_{\frac{1}{2}}$ in the no-scale limit (see also Kane et al. [20]). These point at a correlation between the bounds on the soft parameters. A different class of correlations which take effect in the $h_{t} \rightarrow h_{t}^{\text {fixed }}$ limit is discussed by Carena et al. [37]. Strong correlations (e.g., the $m_{t}^{\text {pole }}-\tan \beta$ correlation $^{9}$, the $h_{t} \rightarrow h_{t}^{\text {fixed }}$ ones, and those due to scalar mixings discussed here) do not allow elimination of the large- $|\mu|$ solutions (that correspond to large left-right mixing enhancement to $m_{h^{0}}$ - see below) on the basis of fine-tuning arguments. The naive notion of fine-tuning becomes obscure and misleading in the presence of strong correlations. (See also Ref. [37].) Rather, strong correlations make the scenario quite predictive.

4. We can rewrite the mixing term in (12) as $h_{t} H_{2}^{0} \hat{\Gamma}$ where $H_{2}^{0}=\nu_{u p} . \hat{\Gamma}$ is strongly constrained by requiring that the full scalar potential does not have a color and/or charge breaking global (GCCB) minimum, which is a different issue than whether the extremum defined by (9) is indeed a minimum. The issue of false vacua is of particular interest in the $\tan \beta \rightarrow 1$ scenario as $V\left(H_{1}, H_{2}\right) \rightarrow 0^{-}$in that limit. Below, we will consider that subject in some detail. In particular, see Appendix B. We find that the absence of negative energy CCB minima implies the constraint, first described in Ref. [43],

$$
\left(\left|A_{t}\right|+s|\mu|\right)^{2} \leq 2\left(m_{\tilde{t}_{L}}^{2}+m_{\tilde{t}_{R}}^{2}\right)
$$

where $s=A_{t} \mu /\left|A_{t} \mu\right|,\left(\left|A_{t}\right|+s|\mu|\right)^{2}=\hat{\Gamma}^{2}$ (for $\tan \beta=1$ ), and $m_{\tilde{t}_{L, R}}^{2}$ consist of only the soft terms. (Note the importance of the trilinear parameter and of the relative sign.) Our numerical studies imply that (13) is (to a good approximation) a sufficient condition (in that region of parameter space) but is not necessary (i.e., it is too restrictive). For example, more than $80 \%$ of the points that are inconsistent with that constraint for $m_{t}^{\text {pole }} \gtrsim 165 \mathrm{GeV}$ correspond to CCB minima which are only local and are therefore safe. In Fig. 2 only filled squares correspond to (probably unacceptable) GCBB minima. Also observe in Fig. 2 that $\mu<0$ is preferred by CCB constraints. This is because typically $A_{t} \gg A_{0}$ for $A_{0}<0$ so that the contribution from a large and positive $\mu$ cannot be canceled in (13) (for our range of $A_{0}$ ).

\section{THE LOOP-INDUCED MASS}

Let us now proceed to discuss the mass of the SM-like higgs boson. The upper bound on $m_{h^{0}}$ is given, e.g., in Ref. [44],

\footnotetext{
${ }^{7}$ The strict no-scale assumption $B_{0}=m_{0}=A_{0}=0$ is not consistent here. See Appendix A.

${ }^{8}$ No-scale models with $h_{b}=h_{\tau}$ were recently discussed in Ref. [42]

${ }^{9}$ Note that even if we do not require $h_{b}=h_{\tau}, m_{t}^{\text {pole }}$ and the lower bound on $\tan \beta$ are correlated due to unitarity considerations.
} 


$$
m_{h^{0}}^{2} \leq M_{Z}^{2} \cos ^{2} 2 \beta+\frac{3 \alpha m_{t}^{4}}{4 \pi s^{2}\left(1-s^{2}\right) M_{Z}^{2}}\left\{\ln \left(\frac{m_{\tilde{t}_{1}}^{2} m_{\tilde{t}_{2}}^{2}}{m_{t}^{4}}\right)+\Delta_{\theta_{t}}\right\}
$$

where

$$
\begin{gathered}
\Delta_{\theta_{t}}=\left(m_{\tilde{t}_{1}}^{2}-m_{\tilde{t}_{2}}^{2}\right) \frac{\sin ^{2} 2 \theta_{t}}{2 m_{t}^{2}} \ln \left(\frac{m_{\tilde{t}_{1}}^{2}}{m_{\tilde{t}_{2}}^{2}}\right) \\
+\left(m_{\tilde{t}_{1}}^{2}-m_{\tilde{t}_{2}}^{2}\right)^{2}\left(\frac{\sin ^{2} 2 \theta_{t}}{4 m_{t}^{2}}\right)^{2}\left[2-\frac{m_{\tilde{t}_{1}}^{2}+m_{\tilde{t}_{2}}^{2}}{m_{\tilde{t}_{1}}^{2}-m_{\tilde{t}_{2}}^{2}} \ln \left(\frac{m_{\tilde{t}_{1}}^{2}}{m_{\tilde{t}_{2}}^{2}}\right)\right],
\end{gathered}
$$

and where $m_{\tilde{t}_{i}}^{2}$ are the eigenvalues of the $t$-scalar mass-squared matrix, $\theta_{t}$ is the mixing angle, and we have neglected other loop contributions. (They are included in our numerical calculations below.) The tree-level mass squared, $m_{h^{0}}{ }^{2}$, and the loop correction, $\Delta_{h^{0}}^{2}$, are bounded by the first and second terms on the r.h.s. of Eq. (14), respectively. In the absence of mixings $\Delta_{\theta_{t}}=0$. For $\tan \beta \rightarrow 1$ one obtains $m_{h^{0}}^{T} \rightarrow 0$, and thus $m_{h^{0}} \approx \Delta_{h^{0}}$.

The upper bound on $\Delta_{h}$ scales as $m_{t}^{4}$ and depends on the overall scale of the $t$-scalar mass, the amount of left-right mixing, and the separation $\left|m_{\tilde{t}_{1}}^{2}-m_{\tilde{t}_{2}}^{2}\right|$. The large mixing and separation for $|\mu| \rightarrow \infty$ enhance $\Delta_{h^{0}}$ significantly (typically by $10-20 \mathrm{GeV}$ ) compared to the first term alone (which was all that was included in Ref. [12]). Condition (2) (and $h_{t}$ unitarity) correlate $m_{t}^{\text {pole }}$ and $\tan \beta$ in case (1) (see Ref. [8,16]), and $\Delta_{\beta}$ increases from $\sim 0$ for $m_{t}^{\text {pole }} \sim 140 \mathrm{GeV}$ to $\sim 1$ for $m_{t}^{\text {pole }} \sim 190 \mathrm{GeV}$. By increasing $\tan \beta$ one gradually departs from the $|\mu| \rightarrow \infty$ limit, and the enhancement to $\Delta_{h^{0}}$ from left-right mixing decreases. Thus, there is an interplay between $\Delta_{\theta_{t}}$ and the overall factor of $m_{t}^{4}$. (Of course, $m_{h^{0}}^{T}$ grows with $\tan \beta$ and hence with $m_{t}^{\text {pole }}$.)

As was pointed out in the previous section (see also Appendix B), $\mu$ and the amount of mixing $\hat{\Gamma}$ are subject to color and/or charge breaking (CCB) constraints. In this section points which are consistent with (13), i.e., which have no negative-energy CCB minima, are marked by a circle - O. Points which are inconsistent with (13) and therefore have either a local or global negative-energy CCB minimum are marked by a "diamond"- $\diamond$. The filled diamonds correspond to a global color breaking minimum (as determined using numerical procedures), which is (most likely) unacceptable. The open diamonds represent CCB minima which are only local (i.e., above the standard model minimum) and are therefore safe. Constraint (13) is always evaluated at the $t$-scalar scale, independent of the scale chosen for minimization. The same is true for the numerical search of global CCB (GCCB) minima.

Calculating $m_{h^{0}}$ is reduced (in our case) to a great extent to calculating $\Delta_{h^{0}}$, i.e., the deviation from what would be the case if the matter were supersymmetric at the weak scale [e.g., $m_{\tilde{t}_{1}}=m_{\tilde{t}_{2}}=m_{t}$ in (14) and (15)]. Two standard ways to perform the calculation are the effective potential method (EPM) (e.g., see Ref. $[45,44,46,36])$ and the renormalization group method (RGM) (e.g., see Ref. [47,48,36]). The two methods correspond to a "run and diagonalize" and "diagonalize and run" algorithm, respectively. The algorithms and their implementations are discussed and compared in greater detail in Appendix C. Here we would like to demonstrate that the EP and RG methods do agree (within their domains of validity), i.e., the results are not sensitive to the way one treats the threshold corrections. In particular, both methods exhibit a large $(10-20 \%)$ two-loop ambiguity from the presence of some twoloop residual terms in the calculations (next-to-leading and leading two-loop logarithms, as well as finite terms). We explicitly identify and discuss such terms in Appendix C. The 
residual logarithmic two-loop terms can be described in terms of a scale ambiguity (i.e., the scale dependence of the one-loop calculation is of two-loop order). Indeed, comparing the two methods and studying that ambiguity allow us to estimate the two-loop correction. However, the uncertainty remains.

In the EPM the ambiguity is explicitly related to a scale dependence, as one is required to specify a subtraction scale $Q$ (i.e., halt running at $Q$ and diagonalize mass matrices). Indeed, including $\Delta V^{\text {one-loop }}(Q)$ corrects for one-loop leading logarithms, and thus guarantees a scale independence of the calculation, but only to that order (and up to wave-function renormalization). It is suggestive to take $Q=M_{Z}$, i.e., the scale at which the physical inputs are given. Another attractive choice is a scale at which $\Delta V^{\text {two-loop }}(Q)$ is minimized. We will not attempt to find such a scale rigorously, but estimate $Q \approx 600 \mathrm{GeV}$. (One indeed expects such a $Q$ to be in the vicinity of some average scalar-quark scale, as for $\Delta V^{\text {one-loop }}$ in Ref. [49].) We will use the comparison between the two methods to justify our choice. (Of course, there is no one choice that is suitable for all sets of parameters.)

In the RGM, which sums the leading logarithms to all orders if one integrates numerically, the ambiguity is more apparent in terms of orders in perturbation theory (i.e., the order at which the summation is truncated in approximate iterative solutions). However, there is also an explicit scale dependence from the choice of decoupling scales for the heavy fields (i.e., decouple heavy eigenstates, calculate RGE's, and run to the next decoupling scale). We will decouple the heavy Higgs doublet $I$ at $m_{A^{0}} \approx 2.1 \mathrm{TeV}$ and other fields at lower thresholds ${ }^{10}$. When decoupling $H$ we include appropriate matching conditions [48].

The EPM is more straightforward to implement (and is therefore more common in the literature) while the RGM requires a careful consideration of decoupling scales and matching conditions.

The scatter plot Fig. 3 displays the results of an EPM calculation with $Q=M_{Z}$. (When increasing $Q$ the soft mass parameters and $m_{t}$ decrease so that the EPM $m_{h}$ is diminished.) The scatter plot Fig. 4 displays the results of a RGM calculation (performed numerically). Comparing them, one observes a $10-20 \mathrm{GeV}$ difference between the respective Higgs boson masses (for a given point in the parameter space). However, this is not due to the different choices of an algorithm, but to the different residual two-loop dependences in the two calculations. To illustrate the maximum ambiguity we used the $t$-quark pole (running) mass in the calculations presented in Fig. 3 (Fig. 4). (The $\sim 5 \%$ difference in the $t$-quark masses is formally of two-loop order.) In both plots one can observe a separation into a heavier and a lighter Higgs boson branches. This is due to the enhancement to $m_{h^{0}}$ in some cases from $\tilde{t}_{L}-\tilde{t}_{R}$ mixing, and is further discussed in the following section.

To further compare the two methods, it is useful to consider the following exercise. The RGM calculation could be modified so that leading logarithms are not summed to all orders, e.g., one could use the one-loop leading-logarithm approximation formula of Ref. [47]. Alternatively, we could minimize the unsummed $\ln \left(m_{\tilde{t}} / Q\right)$ leading (and/or next-toleading) logarithms in the EPM by choosing $Q \approx m_{\tilde{t}}$. In either case the residual terms in the different calculations are qualitatively brought on the same footing. We carried out that

\footnotetext{
${ }^{10}$ We checked that the details of the choices for those intermediate scales introduce a $\sim 1 \%$ uncertainty.
} 
exercise numerically, and Fig. 5 (Fig. 6) compare the EPM with $Q=M_{Z}(Q=600 \mathrm{GeV})$ calculation to the RGM leading logarithm approximation (numerical calculation). Once the residual two-loop dependence of the calculation is properly adjusted, the two algorithms agree well, in particular for $Q \approx 600 \mathrm{GeV}$. [All calculations presented in Fig. 5 (Fig. 6) use the $t$-quark pole (running) mass.]

This all points at the most important source of uncertainty: residual two-loop (and wave-function) scale dependences and a $\sim 5 \% \alpha_{s}$ dependence of $m_{t}$. Two-loop terms fuel the ambiguity, which therefore cannot be removed in a satisfactory fashion within a one-loop calculation. (Even though we could identify some two-loop terms, the consistency of their removal is doubtful). Expanding the calculation to higher orders is not straightforward. We discuss two-loop calculations in Appendix C.

Figs. 3 and 4 define the theoretical ambiguity in determining the loop induced $m_{h^{0}}$ in this scenario. While the former sets the upper bound (for a given $m_{t}^{\text {pole }}$ ), the latter is a more accurate leading-logarithm calculation (and uses properly the running $t$-quark mass). However, within a one-loop calculation, and in particular when next-to-leading logarithms and some finite terms are comparable to higher-loop leading logarithms (which is the case here - see Appendix C), neither method provides a more consistent calculation then the other. As we argue in Appendix C, the former is also an upper bound on twoloop calculations, which most probably diminish $m_{h^{0}}$. We do not think that any stronger statements are justified at present. We continue to discuss the prediction and its upper bound in the next section.

A different issue is that of the constraints on the $t$-scalar left-right mixing enhancement to $\Delta_{h^{0}}$. In the EPM [e.g., Eq. (14)] the enhancement is a straightforward result of diagonalizing the CP-even mass matrix. In the RGM one has to define the decoupling scale for the $t$ scalars. If $m_{\tilde{t}_{1}} \gg m_{\tilde{t}_{2}}$ then one would decouple only $\tilde{t}_{1}$ while $\tilde{t}_{2}$ loops could still contribute to $\Delta_{h^{0}}$. (In practice, the effect is accounted for in the RGM by a set of appropriate matching conditions.) However, the amount of mixing $\hat{\Gamma}$ (and thus the enhancement) is strongly constrained by the physical vacuum.

Requiring that the SM extremum is a minimum (i.e., no negative squared masses corresponding to physical degrees of freedom), and furthermore, is the global minimum (i.e., there is no deeper minimum that does not conserve color and/or charge) strongly constrains $\hat{\Gamma}$. Constraints imposed by the latter may be evaded ${ }^{11}$ if the non-SM global minimum is separated from the SM local minimum by a tunneling time greater than the age of the universe [50]. Constraint (13) (naively) attempts to eliminate all, i.e., local and global, negative-energy CCB minima in that region of parameter space and is too strong. The points marked in the scatter plots by only a an open "diamond" survive a more careful analysis, i.e., they correspond to only local minima ${ }^{12}$. We find, using numerical methods, that for $m_{t}^{\text {pole }} \lesssim 165 \mathrm{GeV}\left(165 \mathrm{GeV} \lesssim m_{t}^{\text {pole }}\right)$ roughly $80 \%(15 \%)$ of the points that fail to

\footnotetext{
${ }^{11}$ This claim, however, does not take into account finite-temperature effects.

${ }^{12}$ In fact, all points are found to have at least a negative-energy CCB local minimum of type $M^{2}=0$ - see Appendix B. However, we find that in our region of the parameter space (13) is a useful tool to identify minima that compete with the standard model minimum.
} 
satisfy constraint (13) correspond to (probably unacceptable) GCCB minima of the (oneloop improved) tree-level scalar potential. The enhancement is then partially washed away by requiring consistency with the physical vacuum. The possibilty of coexistence of the two vacua (with a tunneling time greater than the age of the universe) is, at the present stage of our calculation, a remaining ambiguity. We further discuss those issues in the next section and, in greater detail, in Appendix B.

\section{THE PREDICTION FOR THE HIGGS BOSON MASS AND ITS UPPER BOUND}

In this section we present and discuss our numerical results. In section IV we discussed various methods to calculate the mass (to one-loop). The effective potential method (EPM) with a subtraction scale $Q=M_{Z}$ (e.g., see Fig. 3) puts the weaker constraints on $m_{h^{0}}$ and is the conservative choice for the upper bound. In Fig. 7 we present monte-carlo distributions of $m_{h^{0}}$ using that method for $155 \leq m_{t}^{\text {pole }} \leq 165,165 \leq m_{t}^{\text {pole }} \leq 175$, and $175 \leq m_{t}^{\text {pole }} \leq 185$ $\mathrm{GeV}$. The upper bound can be read off the figures, i.e., $m_{h^{0}} \lesssim 101,106,114 \mathrm{GeV}$ for $m_{t}^{\text {pole } \lesssim}$ $165,175,185 \mathrm{GeV}$, respectively. (Note that these bounds are deduced from our monte-carlo calculations, and are not rigorous.) Nevertheless, we would like to stress that typically $m_{h^{0}}$ lies below its upper bound. Indeed, one cannot rule out the possibility (if $m_{t}^{\text {pole }}$ is heavy enough) that $m_{h^{0}}$ is just outside the range that LEPII may cover. However, this is not likely on both grounds, the $m_{t}^{\text {pole }}$ distribution [Eq. (6)] and the $m_{h^{0}}$ (theoretical) distribution (as reflected in Fig. 7).

The role of the large left-right mixing in the $t$-scalar sector can be seen in Figs. 7a and 7b, which exhibit two peaks in the mass distribution, a "logarithmic" and a "mixing" peak. The former is due to the diagonal entries in the $t$-scalar mass matrix [Eq. (12)] while the latter, which is at larger mass, is due to the mixing enhancement $\left[\Delta_{\theta_{t}}\right.$ in Eq. (15)]. The interplay between the mixing and the $m_{t}^{4}$ factor in the loop correction $\Delta_{h^{0}}$ [e.g., in Eq. (14)], and between $\Delta_{h^{0}}$ and the tree-level mass $m_{h^{0}}^{T}$ (that grows with $\tan \beta$ and, therefore, with $m_{t}^{\text {pole }}$ ) can be seen by comparing Figs. 7a-b with Fig. 7c. The mixing enhancement plays an important role for smaller values of $m_{t}^{\text {pole }}(\Delta \beta \rightarrow 0)$. In fact, that interplay generates for a fixed set of soft parameters a local minimum in the $m_{h^{0}}$ distribution (not shown in the figures) near $m_{t}^{\text {pole }} \approx 165 \mathrm{GeV}$.

The left-right mixing enhancement is strongly constrained when requiring that the minimum of the full scalar potential is the physical one, i.e., no fields other than $H_{1}^{0}=\nu_{\text {down }}$ and $H_{2}^{0}=\nu_{u p}$ have non-vanishing expectation values (see section III and Appendix B). Constraint (13) eliminates potentially dangerous points in the parameter space (marked by a "diamond" in Figs. 3 and 4), some of which, however, correspond to only a local colorbreaking minimum (marked by an open "diamond"). Those which correspond to global minima are marked by a filled "diamond". Fig. 8 is the same as Fig. 7 except that points which correspond to global color and/or charge breaking (GCCB) minima are omitted (the shaded areas have no negative-energy CCB minimum, global or local.) The mixing peak has diminished significantly but has not disappeared. Its presence is even stronger for larger values of $m_{t}^{\text {pole }}$ (see appendix B). (Some caution is required: the cosmological history of the universe may accommodate such a global minimum.) 
A different issue is that of the large two-loop ambiguity in the calculation pointed out in section IV. For example, in Fig. 9 we show the same distributions as in Fig. 7, but in which the running (rather than the pole) $t$-quark mass was used to calculate $m_{h^{0}}$. (The running mass is $\sim 5 \%$ smaller.) Also, the distribution corresponding to the renormalization group method (RGM) calculation (e.g., see Fig. 4), which we showed ${ }^{13}$ in Fig. 6 to roughly agree with the EPM calculation (with $Q \approx m_{\tilde{t}}$ ), is shown in Fig. 10 for $155 \leq m_{t}^{\text {pole }} \leq 165$ and $165 \leq m_{t}^{\text {pole }} \leq 175$, respectively. Again, the $t$-quark running mass is used. Also shown are the distributions when GCCB points are omitted (shaded areas) to be compared with the total areas (shaded and unshaded) in Fig. 8. Note the concentration at lighter masses compared to previous figures, even though the upper limits are only slightly changed. It should be stressed that had we not included the proper RGM matching functions [48] (which would roughly correspond to setting the left-right mixings to zero by hand) then the RGM distribution would sharply peak at the lower end of the $m_{h}$ range. Thus, the importance of the matching functions is obvious.

All, Figs. 7 - 10, are consistent within the theoretical ambiguity. It is suggestive that a consistent two-loop calculation will reduce the upper bounds given by the EPM calculation (we choose conservatively Fig. 7), but probably not to lower values than suggested by the RGM (Fig. 10). Further study is required before any stronger conclusions can be drawn. Even so, for $m_{t}^{\text {pole }} \lesssim 175 \mathrm{GeV} m_{h^{0}}$ [in case (1)] is within the reach of LEPII, and possibly of the Tevatron (see Ref. [51]). If $m_{t}^{\text {pole }}$ is lighter, as suggested by electroweak precision data, our histograms imply that $m_{h^{0}}$ may still be relevant for Higgs searches at LEPI. (In particular, if two-loop corrections reduce $m_{h^{0}}$ as suggested by Fig. 10.)

\section{CONCLUSIONS}

To summarize, we worked in a constrained framework assuming (up to high and low-scale threshold corrections) $\alpha_{1}=\alpha_{2}=\alpha_{3}=\alpha_{G}, h_{b}=h_{\tau}$, and universal initial conditions. Also, we explicitly included the correlation between the $t$-quark mass and the weak angle from electroweak precision data. Within that framework, only $\tan \beta \approx 1$ and large $\tan \beta$ regions are allowed. In the large $\tan \beta$ region (which is allowed only for a large $m_{t}^{\text {pole }}$, unless $m_{b}$ is allowed to get large finite corrections from superpartner loops) $m_{h^{0}}$ is bounded by the triviality bound, and is probably not interesting for Higgs searches at LEPII.

In the $\tan \beta \approx 1$ region the tree-level $m_{h^{0}}^{T}$ nearly vanishes, but grows with $m_{t}^{\text {pole }}$. The loop correction to the mass is enhanced by large $\tilde{t}_{L}-\tilde{t}_{R}$ mixing terms. The latter are diminished with an increasing $m_{t}^{\text {pole }}$ but, as $m_{t}^{\text {pole }}$ grows, the overall factor of $m_{t}^{4}$ can compensate for that effect. The enhancement due to the large mixing is further constrained by requiring the physical vacuum to correspond to the global minimum of the full scalar potential. We find that for parameters corresponding to large mixing there is usually a color breaking minimum, but in many cases this is only a (presumably harmless) local minimum. We also

\footnotetext{
${ }^{13}$ The RGM calculation sums the leading logarithms to all orders in perturbation theory, while in the EPM calculation with $Q \approx m_{\tilde{t}}$ higher order terms are, in principle, minimized, and do not need to be summed.
} 
pointed out that a two-loop ambiguity in the calculation of the Higgs boson mass is enhanced in our case and can be as large as $20 \mathrm{GeV}$. All these effects lead to the conservative bound $m_{h^{0}} \lesssim 100(110) \mathrm{GeV}$ for $m_{t}^{\text {pole }} \lesssim 160$ (175) GeV. (We briefly discuss two-loop calculations in Appendix $\mathrm{C}$, where we conclude that such a calculation will most probably strengthen the upper bound.) The Higgs boson mass in the class of models characterized by $\tan \beta \approx 1$ is then almost certainly within the reach of LEPII (and possibly within the reach of the Tevatron [51]). Furthermore, the Higgs boson is typically lighter than the upper bound, and may be light enough to still be discovered at LEPI.

Regarding the $t$-quark mass, one requires for $S U(2) \times U(1)$ breaking (in this scenario) $m_{t}^{\text {pole }} \gtrsim 140 \mathrm{GeV}$, and further requiring $\tan \beta \gtrsim 1.1$ (which is the case if the divergent limit is to be avoided, i.e., if the divergence is not stabilized by model-dependent finite terms) we obtain $m_{t}^{\text {pole }} \gtrsim 155 \mathrm{GeV}$. Eq. (6) and, in particular, the structure of the radiative corrections

to the $Z \rightarrow b \bar{b}$ vertex, however, suggest that $m_{t}^{\text {pole }}$ is not much larger. (The latter can be evaded only if both the $t$-scalar and the Higgsino are light, which is not very likely in our case.) Also, we were able to understand the smallness of the parameter space allowed by $h_{b}=h_{\tau}$ in terms of a custodial symmetry. The symmetry (theoretical) argument suggests $m_{t}^{\text {pole }} \lesssim 185 \mathrm{GeV}\left(\Delta_{\beta} \lesssim 1\right)$. In Appendix D we briefly examine extended Higgs sectors, and point out that the Higgs mass is a possible probe of such extensions if $m_{t}^{\text {pole }}(\mathrm{and} / \mathrm{or} \tan \beta)$ is known.

In Appendix B we point out that the full (one-loop improved) scalar potential generically possesses color and/or charge breaking negative-energy local minima. Analytic constraints that are often found in the literature attempt to eliminate all such local minima (i.e., are too strong) but by construction apply to only certain types of minima while ignoring others (i.e., are too weak). We give a general classification of the local minima and are able to further identify [in case (1)] those that can potentially compete with the standard model minimum.

\section{ACKNOWLEDGMENTS}

This work was supported by the US Department of Energy Grant No. DE-AC02-76ERO-3071. It is pleasure to thank J. Erler for useful discussions concerning electroweak observeables, and to A. Pomarol for useful discussions and for his comments on the manuscript.

\section{APPENDIX A: THE HIGGS SECTOR CUSTODIAL SYMMETRIES}

In this appendix we examine some symmetry aspects of the SGUT solutions, and in particular, of case (1). Some features of the latter, as well as the distinction between the two cases, can be better understood in terms of the custodial $S U(2)_{L+R}$ approximate symmetry of the SM lagrangian [26]; i.e., turning off hypercharge and flavor mixings, and if $h_{t}=h_{b}=h$, then one can rewrite the $t$ and $b$ Yukawa terms as an $S U(2)_{L} \times S U(2)_{R}$ invariant, i.e.,

$$
h\left(\begin{array}{c}
t_{L} \\
b_{L}
\end{array}\right)_{a} \epsilon_{a b}\left(\begin{array}{cc}
H_{1}^{0} & H_{2}^{+} \\
H_{1}^{-} & H_{2}^{0}
\end{array}\right)_{b c}\left(\begin{array}{c}
-b_{L}^{c} \\
t_{L}^{c}
\end{array}\right)_{c}
$$


(where in the SM $H_{2}=i \tau_{2} H_{1}^{*}$ ) and $S U(2)_{L} \times S U(2)_{R} \rightarrow S U(2)_{L+R}$ for $\nu \neq 0$. However, $h_{t} \neq h_{b}$ and the different hypercharges of $t_{L}^{c}$ and $b_{L}^{c}$ explicitly break the left-right symmetry, and therefore the residual custodial symmetry.

In the MSSM, on the other hand, $H_{1}$ is distinct from $H_{2}$ and if $\nu_{\text {down }} \neq \nu_{\text {up }} S U(2)_{L} \times$ $S U(2)_{R} \rightarrow U(1)_{T_{3 L}+T_{3 R}}[27]$. In particular, the $S U(2)_{L+R}$ symmetry is preserved if $\beta=\frac{\pi}{4}$ but is maximally broken if $\beta=\frac{\pi}{2}$. Ignoring hypercharge, we can then define for illustration two quantities,

$$
\begin{aligned}
B_{M} & \equiv \nu_{\text {down }} \frac{h_{t}-h_{b}}{m_{t}-m_{b}}, \\
B_{H} & \equiv m_{b} \frac{\tan \beta-1}{m_{t}-m_{b}},
\end{aligned}
$$

that measure the $S U(2)_{L+R}$ symmetry breaking in the matter and Higgs sectors, respectively. If we map the $\tan \beta-m_{t}^{\text {pole }}$ plane onto the $B_{M}-B_{H}$ plane, we find that cases (1) and (2) approximately correspond to $(1,0)$ and $(0,1)$, respectively. Thus, while the constrained $S O(10)$ solution realizes $m_{t} \neq m_{b}$ as an intrinsic property of the vacuum (i.e., $\nu_{u p} \gg \nu_{\text {down }}$ ) and the grand-unified symmetry protects $B_{M} \approx 0[29]$, the $\tan \beta \approx 1$ case realizes the $S U(2)_{L+R}$ (explicit) breaking as a scaling phenomenon (i.e., $B_{M} \propto h_{t}^{\text {fixed }}$ ) and the vacuum respects the symmetry (up to loop corrections).

The symmetry that is spontaneously broken in case (2) is a $O_{4} \times O_{4}$ symmetry. Haber and Pomarol observed [28] that for $m_{3} \rightarrow 0$ [which is the situation in case (2)] there is no mixing between $\mathrm{H}_{1}$ and $\mathrm{H}_{2}$ and the Higgs sector respects $\mathrm{O}_{4} \times \mathrm{O}_{4}$ (up to gauge-coupling corrections). The symmetry is broken to $O_{3} \times O_{3}$ for $\nu_{\text {down }} \neq \nu_{u p} \neq 0$ and the six Goldstone bosons are the three SM Goldstone bosons, $A^{0}$, and $H^{ \pm}$. The symmetry is explicitly broken for $g_{2} \neq 0$ (so $m_{H^{+}}=M_{W}$ ) and is not exact even when neglecting gauge couplings (i.e., $m_{3} \neq 0$ ). Thus, $A^{0}$ and $H^{ \pm}$are massive pseudo-Goldstone bosons, i.e., $m_{H^{+}}^{2}-M_{W}^{2} \approx m_{A^{0}}^{2}=C \times m_{3}^{2}$. However, $C=-\frac{2}{\sin 2 \beta}$ and can be large, which is a manifestation of the fact that $O_{4} \times O_{4} \rightarrow O_{4} \times O_{3}$ for $\nu_{\text {down }}=0$. (The limit $m_{3} \rightarrow 0$ corresponds also to a $U(1)$ Peccei-Quinn symmetry [13].)

Our main interest in this work is, however, case (1), where from (9b) the Higgs-sector $S U(2)$ custodial symmetry constrains

$$
m_{H_{1}}^{2}+\mu^{2}=m_{H_{2}}^{2}+\mu^{2}=-m_{3}^{2} .
$$

This is realized by taking the limit $|\mu| \rightarrow \infty$, provided $|B| \approx|\mu|$ which in our formalism comes out as a prediction. (Indeed, many SGUT models can easily realize a large $B_{0}$, for example, see Ref. [52].) Relation (A3) results in a (tree-level) massless eigenvalue in the neutral CP-even mass matrix. (Note that the SM Goldstone bosons are also massless Goldstone bosons of the broken $S U(2) \times S U(2)$.)

The smallness of $\Delta_{\beta}$ is protected by the custodial symmetry, i.e., $\Delta_{\beta} \neq 0$ is explained by loop corrections. The broken symmetry in the Yukawa sector induces at the loop level a small breaking in the Higgs sector (e.g., $m_{H_{1}}^{2} \neq m_{H_{2}}^{2}$ ). The magnitude of the effect can be estimated by integrating $d \tan \beta / \tan \beta$, i.e.,

$$
\Delta_{\beta}\left(M_{Z}\right)=\Delta_{\beta}\left(M_{G}\right)-\frac{3}{16 \pi^{2}} \int_{\ln \frac{M_{G}}{M_{Z}}}^{0}\left(h_{t}^{2}-h_{b}^{2}\right) d \ln \frac{Q}{M_{Z}} \approx \Delta_{\beta}\left(M_{G}\right)+0.6,
$$


where we neglected $h_{\tau}$, as well as threshold corrections, took $h_{t} \approx 1, h_{b} \approx 0$, and assumed $\Delta_{\beta}<1$. Thus, even though the parameter space in case (1) is severely restricted, we can understand the restriction in terms of a symmetry.

\section{APPENDIX B: CONSTRAINTS ON COLOR AND CHARGE BREAKING MINIMA}

Let us consider the super-potential

$$
W=h_{t} \tilde{t}_{L} \tilde{t}_{R} H_{2}^{0}+\mu H_{1}^{0} H_{2}^{0},
$$

where we have performed an $S U(2)$ rotation so that $H_{2}^{+}$has no vacuum expectation value. (We do not distinguish our notation for a superfield from that of its scalar component.) It is easy to convince oneself that only those terms in the super-potential are relevant when

searching for the global minimum of the scalar potential in the large $h_{t}$ limit [53]. Using standard techniques we arrive at the corresponding scalar potential:

$$
h_{t}^{2} V=M^{2}-\Gamma+\Lambda
$$

where the bilinear, trilinear, and quartic terms are

$$
\begin{gathered}
M^{2}=m_{1}^{2} H_{1}^{0^{2}}+m_{2}^{2} H_{2}^{0^{2}}+2 m_{3}^{2} H_{1}^{0} H_{2}^{0}+m_{\tilde{t}_{L}}^{2} \tilde{t}_{L}^{2}+m_{\tilde{t}_{R}}^{2} \tilde{t}_{R}^{2}, \\
\Gamma=\left|2\left(\left|A_{t}\right| H_{2}^{0}+s|\mu| H_{1}^{0}\right) \tilde{t}_{L} \tilde{t}_{R}\right|,
\end{gathered}
$$

and

$$
\Lambda=\left(\tilde{t}_{L}^{2}+\tilde{t}_{R}^{2}\right) H_{2}^{0^{2}}+\tilde{t}_{L}^{2} \tilde{t}_{R}^{2}+\frac{1}{h_{t}^{2}}\left(\frac{\pi}{2} \times “ D-\text { terms" }\right),
$$

respectively. All fields were scaled $\phi \rightarrow \phi / h_{t}$ and are taken to be real and positive (our phase choice for the fields, which fixed $m_{3}^{2}<0$ and $\Gamma>0$, i.e., maximized the negative contributions to $V$ ) and all parameters are real. $m_{1,2}^{2}=m_{H_{1,2}}^{2}+\mu^{2}, s=\mu A_{t} /\left|\mu A_{t}\right|$, and the expression for the "D-terms" is [53]

$$
\frac{12}{5} \alpha_{1}\left[-\frac{H_{1}^{0^{2}}}{2}+\frac{H_{2}^{0^{2}}}{2}+\frac{\tilde{t}_{L}^{2}}{6}-\frac{2 \tilde{t}_{R}^{2}}{3}\right]^{2}+\alpha_{2}\left[H_{1}^{0^{2}}-H_{2}^{0^{2}}+\tilde{t}_{L}^{2}\right]^{2}+\frac{4}{3} \alpha_{3}\left[\tilde{t}_{L}^{2}-\tilde{t}_{R}^{2}\right]^{2} .
$$

We can parametrize the four fields in terms of an overall scale $X$ and three angles $0 \leq$ $\alpha, \beta, \gamma \leq \frac{\pi}{2}: H_{1}^{0}=X \sin \alpha \cos \beta, H_{2}^{0}=X \sin \alpha \sin \beta, \tilde{t}_{R}=X \cos \alpha \cos \gamma, \tilde{t}_{L}=X \cos \alpha \sin \gamma$, and redefine

$$
h_{t}^{2} V(X)=M^{2}(\alpha, \beta, \gamma) X^{2}-\Gamma(\alpha, \beta, \gamma) X^{3}+\Lambda(\alpha, \beta, \gamma) X^{4}
$$

Then, for fixed angles, $V(X)$ will have a minimum for $X \neq 0$ provided the condition $32 M^{2} \Lambda<9 \Gamma^{2}$ is satisfied. In that case, 


$$
X_{\text {min }}=\frac{3}{8} \frac{\Gamma}{\Lambda}\left[1+\left(1-\frac{32 M^{2} \Lambda}{9 \Gamma^{2}}\right)^{\frac{1}{2}}\right] \geq 0
$$

and

$$
h_{t}^{2} V_{\min }=-\frac{1}{2} X_{\min }^{2}\left(\frac{\Gamma}{2} X_{\min }-M^{2}\right)
$$

We only allow parameters for which a standard model (SM) minimum exists. The SM minimum corresponds to $\alpha=\frac{\pi}{2}$ and $\beta=\beta^{0}\left(\gamma\right.$ is irrelevant and $\tan \beta^{0}=\nu_{\text {up }} / \nu_{\text {down }}$ is the angle used to fix $\mu, m_{3}$, as well as the Yukawa couplings). It is easy to convince oneself that in that limit the $4 \times 4$ second-derivative matrix is $2 \times 2$ block diagonal (otherwise baryon number is violated). Thus, it is sufficient to confirm that the four physical eigenvalues are positive to ensure that it is a minimum. (This is done in our numerical calculations.) If these conditions are satisfied then the SM is at least a local (negative-energy) minimum, and one has $\Gamma_{\mathrm{SM}}=0, M_{\mathrm{SM}}^{2}<0$, and (B8) and (B9) reduce to the usual results $X_{\text {min }}^{\mathrm{SM}}=\sqrt{-M_{\mathrm{SM}}^{2} / 2 \Lambda_{\mathrm{SM}}}$, $h_{t}^{2} V_{\min }^{\mathrm{SM}}=-M_{\mathrm{SM}}^{4} / 4 \Lambda_{\mathrm{SM}}$.

Let us now consider the possibility of additional CCB minima with $\cos \alpha \neq 0$. Since $m_{\tilde{t}_{L, R}}^{2}>0$ for the class of models we are considering this requires $\Gamma \neq 0$, which we assume hereafter. From (B7) - (B9) it is easy to classify the possible color and/or charge breaking (CCB) minima for definite $\alpha, \beta, \gamma$. One finds that for $\Gamma^{2} \leq 32 \Lambda M^{2} / 9$ there is no CCB minimum, while for $32 \Lambda M^{2} / 9<\Gamma^{2} \leq 4 \Lambda M^{2}$ the CCB minimum exists but has $V_{\min }^{\mathrm{CCB}}>0>$ $V_{\text {min }}^{\text {SM }}$, which is presumably safe. For $4 \Lambda M^{2}<\Gamma^{2}$ (including the more rare case $M^{2}<0$, that must fall in this category) there is a negative-value CCB minimum, which may however be either local (presumably safe), i.e., $V_{\text {min }}^{\mathrm{CCB}}>V_{\text {min }}^{\text {sM }}$, or global (probably unacceptable), i.e., $V_{\min }^{\mathrm{CCB}}<V_{\min }^{\mathrm{SM}}$. Here, a sufficient (but not necessary) condition for an acceptable model is

$$
\Gamma^{2} \leq 4 \Lambda M^{2}
$$

which generalizes that of Ref. [53]. In principle, the above discussion holds for any number of fields (i.e., any number of angles), only the explicit expressions for $M^{2}, \Gamma$, and $\Lambda$ are more complicated. If constraint (B10) holds for every choice of $\alpha, \beta$, and $\gamma(\cos \alpha \neq 0)$ then there is no negative-valued color and/or charge breaking minimum, global (GCCB) or local. In the special case $M^{2}<0$ the constraint cannot be satisfied and there will be a negative-energy local CCB minimum, an observation made previously by Gunion et al. [53].

If (B10) does not hold, further investigation is needed to determine whether the minimum is global or local. It is straightforward to show that (B10) is satisfied for $\sin \beta=0$, i.e., there is no negative-valued CCB minimum for $H_{2}^{0}=0$ for the class of models we are considering. We will therefore restrict our attention to the case $H_{2}^{0} \neq 0$, in which case it is convenient to reparametrize $X=H_{2}^{0}$ and rescale all fields by an additional factor of $1 / H_{2}^{0}$. This simplifies the discussion and enables us to examine and generalize previous work on the subject $[54,43,55,53]$.

By inspection (B10) cannot hold for all values of the fields and there is always (and not just for $M^{2}<0$, which is hard to achieve in dangerous directions) a local CCB minimum, e.g., for $M^{2}=0$ ( $\Lambda$ is positive definite). For example, one can choose

$$
H_{1}^{0}=-\frac{m_{3}^{2}}{m_{1}^{2}},
$$




$$
\begin{gathered}
\tilde{t}_{L}=\frac{1}{m_{\tilde{t}_{L}}} \sqrt{\frac{1}{2}\left(\frac{m_{3}^{4}}{m_{1}^{2}}-m_{2}^{2}\right)}, \\
\tilde{t}_{R}=\tilde{t}_{L} \frac{m_{\tilde{t}_{L}}}{m_{\tilde{t}_{R}}},
\end{gathered}
$$

which is always possible because of (8). We confirmed this in our numerical studies, i.e., there is a CCB (negative-valued) local minimum even if $V^{\mathrm{SM}}=V\left(\nu_{\text {down }}, \nu_{\text {up }}\right)$ is the global minimum. (We do not consider additive constants.) Although such local minima may be of cosmological interest, the relevant question in our case is whether $V^{\mathrm{SM}}$ is the global minimum of the full (or more precisely, of the four-field) scalar potential. Similarly, we assume that positive-valued CCB minima would not be populated and are therefore harmless. The answer has to be given by numerical mapping of all minima, but it is still useful to review how one could derive analytic constraints from (B10), which are typically relevant only for specific regions of the parameter space.

For example, if we fix $H_{1}^{0}=0, \tilde{t}_{L}=\tilde{t}_{R}=1$, then (B10) gives the well known result [54]

$$
A_{t}^{2} \leq 3\left(m_{\tilde{t}_{L}}^{2}+m_{\tilde{t}_{R}}^{2}+m_{H_{2}}^{2}+\mu^{2}\right)
$$

The equal field direction was chosen so the positive $D$-term contribution $\propto 1 / h_{t}^{2}$ vanishes. This is not a relevant requirement for $h_{t} \approx 1$ (i.e., there may exist a deeper minimum with non vanishing $D$-terms). It is obvious that (B12) is not relevant when $|\mu| \rightarrow \infty$. We find that a more useful constraint is [taking $H_{1}^{0} \approx 1, \tilde{t}_{L} \approx \tilde{t}_{R} \equiv t \ll 1$ and using $(\mathrm{B} 10)$ ]

$$
\left(\left|A_{t}\right|+s|\mu|\right)^{2} \leq 2\left(m_{\tilde{t}_{L}}^{2}+m_{\tilde{t}_{R}}^{2}\right) .
$$

Note that if the order $t^{2}$ corrections to (B13) are not negligible then $V$ is less negative, which motivated our choice $t \ll 1$, i.e., the more dangerous direction. That constraint was also used by Drees et al. [43] to describe their numerical results (i.e., if we take $m_{1}=m_{2}$ in their formula). We confirmed in our numerical studies that (B13) and not (B12) is relevant in our case. [We found nearly all points to be consistent with (B12) for our ranges of the soft parameters.]

Finally, we perform numerical minimization of the potential (B2) and map all minima using monte-carlo routines. We find that (B13) is to a good approximation sufficient to avoid a GCCB minimum, but is not necessary. For example, only about $80 \%$ (15\%) of the points which are inconsistent with (B13) correspond to GCCB for $m_{t}^{\text {pole }} \lesssim 165 \mathrm{GeV}$ $\left(m_{t}^{\text {pole }} \gtrsim 165 \mathrm{GeV}\right)$. We compare the points consistent with (B13) and with the numerical search in Fig. 8. The greater relevance of (B13) for $m_{t}^{\text {pole }} \lesssim 165 \mathrm{GeV}$ can be understood if we recall that the lower bound on $|\cos 2 \beta|$ increases with $m_{t}^{\text {pole }}$, and thus $V^{s \mathrm{~m}}$ becomes a deeper minimum. (The lower bound is related to $h_{t}$ unitarity, and that statement is independent of our $h_{b}=h_{\tau}$ assumption.) On the other hand, $|\mu|$ and the negative terms in $V^{\mathrm{CCB}}$ [e.g., Eq. (B2)] diminish with increasing $m_{t}^{\text {pole }}$. That interplay shifts the CCB minimum from a global to a local minimum.

Let us demonstrate that claim in a simple-minded example assuming $h_{t} \approx h_{t}^{\text {fixed }} \approx 1-1.1$. We define $\sin ^{2} \beta=2 m_{t}^{2} / h_{t}^{2} \nu^{2} \equiv x, 0.5 \leq x \leq 1\left[\nu=2 M_{W} / g_{2}\right]$. We can rewrite 


$$
h_{t}^{2} V^{\mathrm{SM}}=-\frac{1}{8} M_{Z}^{2} h_{t}^{2} \nu^{2}[1-4 x(1-x)]
$$

$\left|h_{t}^{2} V^{s \mathrm{M}}\right|$ sharply increases with $m_{t}^{\text {pole }}$ (i.e., with $x$ ). On the other hand, taking $m_{H_{2}}^{2} \approx M_{Z}^{2} \approx 0$ in $(9 \mathrm{a})$ we have

$$
\mu^{2} \approx m_{H_{1}}^{2}\left(\frac{1-x}{2 x-1}\right)
$$

and $\mu^{2}$ decreases from $+\infty$ to 0 in the above range of $x$. Now assume that constraint (B13) is not satisfied, i.e.,

$$
h_{t}^{2} V^{\mathrm{CCB}} \approx-\frac{1}{2} \kappa^{2} \mu^{2}\left(\kappa \mu^{2}-m_{\tilde{t}_{L}}^{2}-m_{\tilde{t}_{R}}^{2}\right) t^{2}
$$

where $t^{4} \ll t^{2}$ and $\kappa$ is a positive number in the range $\left[\frac{3}{8}, \frac{3}{4}\right]\left(X_{\min }=\kappa|\mu|\right)$. We also assumed $\left|A_{t}\right| \ll|\mu|$, and neglected $t^{4}$ terms. $\left(M^{2}<0\right.$ would increase $\kappa$.) To further simplify, consider the case $m_{0}=0$, so that $m_{\tilde{t}_{L}}^{2} \approx m_{\tilde{t}_{R}}^{2} \approx 6 M_{\frac{1}{2}}^{2} \approx 12 m_{H_{1}}^{2} \cdot V^{\mathrm{CCB}} \lesssim V^{S M}$ in this case if

$$
\frac{1}{4 \kappa^{2}} \frac{M_{Z}^{2} h_{t}^{2} \nu^{2}}{m_{H_{1}}^{4} t^{2}} \lesssim\left(\frac{1-x}{2 x-1}\right) \frac{\left[\kappa\left(\frac{1-x}{2 x-1}\right)-24\right]}{1-4 x(1-x)}
$$

The r.h.s. has to be positive, i.e., $x \rightarrow 0.5^{+}$or $m_{t}^{\text {pole }} \lesssim 150 \mathrm{GeV}$. Slightly increasing $m_{t}^{\text {pole }}$ would reverse the inequality.

Lastly, there are two caveats. The first is that we used in this section the (one-loop improved) tree-level potential. However, following Ref. [49] we perform the calculations at the $t$-scalar scale to eliminate large loop corrections. Secondly, a GCCB minimum may be "safe" if separated from the local standard-model minimum by a tunneling time greater than the age of the universe [50]. Such considerations are beyond the scope of our present work and would also require a consideration of finite-temperature effects.

\section{APPENDIX C: CALCULATION OF THE LOOP-INDUCED MASS}

\section{The EPM: Run and diagonalize}

A straightforward way to calculate $\Delta_{h^{0}}$ is $(a)$ to take the first and second derivatives of $\Delta V(Q)$ with respect to the neutral $\mathrm{CP}$-even and $\mathrm{CP}$-odd components of $H_{i}$ at the minimum; (b) to absorb the latter in $m_{A^{0}}$; and (c) to calculate the correction to (11): the effective potential method (EPM). Like Ref. [44], we will follow Ref. [45], however, we add to their expressions "D-term" [46] and Higgs-Higgsino and gauge-gaugino [36] contributions, which are typically $-(1-2)$ and $-(2-4) \mathrm{GeV}$, respectively. Note that $(i)$ all the parameters are taken at the subtraction scale $Q$. The mass matrices are thus calculated at the scale $Q$ and (ii) only then are diagonalized, i.e., a "run and diagonalize" algorithm. Our two choices in section IV were $Q=M_{Z}$ and $Q=600 \mathrm{GeV} \sim m_{\tilde{t}}$. (Large $\ln \frac{m_{A^{0}}}{M_{Z}} \operatorname{logarithms}$ are multiplied by small couplings and do not invalidate the loop expansion, i.e., the EPM. This is equivalent to the statement above that Higgs-Higgsino contributions are small.) One 
corrects for threshold effects by the subtraction of one-loop leading logarithms (included in $\Delta V)$, and thus to that order the two choices are equivalent.

The $Q$ dependence of $m_{h^{0}}(Q)$ comes about from $(i)$ logarithmic wave-function dependence of $\nu_{i}(Q)$ and (ii) two-loop implicit field-independent dependences of $\Delta V(Q)=$ $\Delta V\left[m_{i}(Q), \alpha_{i}(Q), h_{t}(Q)\right]$ (where $m_{i}$ runs over the relevant mass parameters). Both are often underestimated. The importance of $(i)$ is obvious, e.g., from the $m_{t}^{4} / M_{Z}^{2}$ factor. The field-independent dependence (ii) was studied in Ref. [56], where it was shown to be of the order of two-loop (next-to-leading) logarithms. One can instead examine directly the expression for $\Delta_{h^{0}}$, e.g., the leading logarithm in (14) evolves with scale (neglecting $\Delta_{\theta_{t}}$ and multiplicative factors) roughly as

$$
\frac{1}{8 \pi^{2}} \ln \left(\frac{m_{\tilde{t}}^{2}\left(Q^{2}\right)}{m_{t}^{2}}\right) \approx \frac{1}{8 \pi^{2}} \ln \left(\frac{m_{\tilde{t}}^{2}\left(Q_{0}^{2}\right)}{m_{t}^{2}}\right)+\frac{1}{128 \pi^{4}}\left\{\kappa h_{t}^{2}\left[\frac{m_{H_{2}}^{2}+A_{t}^{2}}{m_{\tilde{t}}^{2}}+2\right]-\frac{16}{3} g_{s}^{2} \frac{M_{\tilde{\hat{\theta}}}^{2}}{m_{\tilde{t}}^{2}}\right\} \ln \frac{Q^{2}}{Q_{0}^{2}},
$$

where $\kappa=1,2$ for $\tilde{t}_{L, R}$, respectively, and we took $m_{t}(Q) \approx m_{t}\left(Q_{0}\right)$, etc., $m_{\tilde{t}_{L}} \approx m_{\tilde{t}_{R}}$, and neglected all terms aside from $h_{t}$ and $g_{s}^{2}\left(=4 \pi \alpha_{s}\right)$ ones. ( $M_{\tilde{g}}$ is the gluino mass.) This is of course a rough estimate, as, in practice all the parameters scale with $Q$. However, it illustrates qualitatively the presence of residual two-loop logarithms which are enhanced by the large couplings and masses when evaluating $\Delta_{h^{0}}$. Note that typically the QCD term wins and the correction is positive near $Q=M_{Z}$.

Though formally of two-loop order, the residual $Q$ dependence of the EPM $m_{h^{0}}$ is (in our case) of order $10-20 \%$. The ambiguity in $m_{h^{0}}$ is reduced for $m_{h^{0}}^{T} \gg 0$. Also, for values of $\tan \beta$ outside the allowed region $\Delta_{\theta_{t}}$ is diminished (e.g., in the notation of Ref. [45] only $\Delta_{22}$ is important), $h_{t}<1$ (which also diminishes the wave-function renormalization), and thus, there is a smaller ambiguity not only in $m_{h^{0}}$ but also in $\Delta_{h^{0}}$. Nevertheless, one should be aware of such ambiguities, which are generically present in a calculation of that sort.

\section{The RGM: Diagonalize and run}

One could alternatively correct for thresholds by explicitly decoupling a particle below its threshold and redefining the effective field theory: the renormalization group method (RGM). Following Ref. [36,47], we diagonalize the Higgs mass matrices at scale $Q=m_{A^{0}}$, integrate out the heavy Higgs doublet, and define a one Higgs doublet model (1HDM) effective theory with a quartic coupling $\frac{\lambda}{2} h^{4}$. Note that $m_{A^{0}} \gg m_{\tilde{t}}$, so only the heavy Higgs doublet is integrated out at $Q=m_{A^{0}}$, and other heavy particles will be decoupled at lower thresholds. The boundary condition for $\lambda\left(m_{A^{0}}\right)$ is determined by the MSSM Higgs couplings at that scale and requires knowledge of $\tan \beta\left(m_{A^{0}}\right)$ (i.e., wave-function renormalization). Mixing effects are accounted for by a complicated set of matching conditions [48,47]. $\lambda$ is then evolved down to $M_{Z}$, where $m_{h^{0}}=\sqrt{\lambda} \nu=2 \sqrt{\lambda} M_{W} / g_{2}$; i.e., a "diagonalize and run" algorithm.

One can solve the RGE's iteratively to get a semi-analytic approximation [36,47]. This is not an exact procedure, and at one-loop is accurate to leading logarithms (i.e., the accuracy of the EPM). Alternatively, if the RGM calculation is done by exact (or numerical) integration, then the leading logarithms are summed to all orders. In agreement with Ref. [36] we find that $\lambda\left(M_{Z}\right)$ (and thus $m_{h^{0}}$ ) is diminished when integrating the RGE's numerically. However, the effect of the numerical integration (Fig. 5) is more important than anticipated 
in Ref. [36] since $A^{0}$ is heavier than the mass-scale used there and because of the large couplings. The difference between the numerical treatment and the approximate formula is of order of two-loop (leading) logarithms. The summation of the leading logarithms modifies the scale dependence of the calculated mass.

A different scale dependence is via the details of the decoupling of heavy states. To ease the calculation, we do not diagonalize scalar-quark mass-squared matrices at an intermediate scale. Instead, we put in the appropriate matching conditions [48,47] by hand. These conditions are derived by expanding the EP assuming $\left|m_{t} \mu\right| \lesssim m_{\tilde{t_{I}}}^{2}$, etc., which still holds in our case $\left[m_{t}\left(m_{A^{0}}\right)<m_{t}\left(M_{Z}\right)\right]$. Nevertheless, we repeated the 1HDM RGM calculation but using the EPM with $Q \approx m_{A^{0}}$ to determine $m_{h^{0}}\left(m_{A^{0}}\right)$, and thus [using $\nu\left(m_{A^{0}}\right)$ ] the boundary condition for $\lambda\left(m_{A^{0}}\right)$. The agreement with the calculation using the boundary conditions of Ref. $[48,47]$ is good.

\section{Validity of the comparison between the EPM and RGM}

Whether "running and diagonalizing" or vice versa leads to a slightly different answer. However, it was already noted in Ref. [36] that the difference between the two algorithms is small and of higher order, and can be ignored for the purpose of our discussion here. However, the inclusion of the appropriate RGM matching conditions is essential for the comparison between the two methods.

More importantly, the RGM approximation of 1HDM breaks down if $|\mu|$ is not sufficiently large. (In such cases the more conventional 2HDM RGM is appropriate.) Too small scalarquark (diagonal) masses break down the expansion of Ref. [48,47] and too large scalar threshold-corrections can break down the EPM approximation. Indeed, one has to ensure that the scalar quarks are not heavier than about $1 \mathrm{TeV}$ in order not to invalidate the loop expansion which underlies the EP, as these large logarithms would be multiplied by large couplings ${ }^{14}$ (see, for example, Ref. [57]). We checked that the points in the parameter space where the RGM and EPM are in sharp disagreement (e.g., in Figs. 5 and 6) correspond to either one of these cases.

\section{Two-loop calculations}

In this work we do not carry out a two-loop calculation of $\Delta_{h^{0}}$. Such a calculation is an elaborate task and attention should be paid to various issues, e.g., wave-function renormalization and the coupled running of the masses and couplings; pole masses vs. running masses; the multi-scale structure of the low-energy theory; the correct choice of matching conditions (logarithmic and non-logarithmic) given a choice of decoupling scales; one-loop finite pieces (not included in the EP) that may become important in certain regions of the parameter space; in addition to correct counting of powers of the couplings. In fact, an hybrid algorithm between the EPM and RGM is required.

\footnotetext{
${ }^{14}$ This requirement is consistent with our choice of ranges for the soft parameters.
} 
We received recently two interesting papers where (partial) two-loop analyses were presented $[58,59]$. In this section we compare those results with the residual two-loop terms we identified above. We will take a somewhat critical point of view emphasizing that though the above studies (as well as the discussion here) pave the way to understanding $\Delta_{h^{0}}$ beyond one-loop order, they are not complete. We conclude that the one-loop leading-logarithm result is most probably an upper bound on the two-loop result, but we doubt whether any stronger conclusions can be drawn at the present.

Above, we presented the leading-logarithm result. In the EPM this is given by $\Delta V^{\text {one-loop }}$ and in the RGM one solves the RGE's to that order. We found no significant difference between the two methods. Next, we proceeded to calculate beyond the leading order and integrated the RGM RGE's numerically, summing the leading logarithms to all orders in perturbation theory, and, in particular, to two-loop order. We then found a $\sim-10-20 \%$ correction to $\Delta_{h^{0}}$. On the other hand, we identified in the EPM expressions next-to-leading two-loop logarithmic terms. Those terms come about from the scale dependence of the running parameters, and introduce a similar (but positive) correction to $\Delta_{h^{0}}$. Also, we noted the important $(\sim 10 \%)$ effect of $m_{t}^{\text {pole }} / m_{t}^{\text {running }} \approx\left[1+\left(4 \alpha_{s} / 3 \pi\right)\right]$ [e.g., $\left(m_{t}^{\text {running }}\right)^{4} \approx$ $\left.0.8\left(m_{t}^{\text {pole }}\right)^{4}\right]$. It is then suggestive that the two-loop leading logarithms and $m_{t}$ corrections would diminish the one-loop (leading-logarithm) result, while the next-to-leading logarithms would partially counter-balance that effect. Thus, we reach the conclusion that the one-loop leading-logarithm prediction for $m_{h^{0}}$ (which contain some next-to-leading logarithms) is an upper bound on the two-loop prediction. Any stronger conclusion would require a detailed consideration of the issues listed above. Furthermore, the significance of a detailed twoloop study in the context of SGUT's is not clear unless complemented by similar studies of threshold effects near the high-scale boundary.

Let us proceed with the issue of the orders in perturbation theory. It was already pointed out by Chankowski [57] that the inclusion of $\Delta V^{\text {one-loop }}$ in (7) with only one-loop RGE's for the (RG-improved) potential parameters is an inconsistent procedure when counting powers of couplings. These issues have recently received great attention in the context of theories with a single scalar field (i.e., $\phi^{4}$ or the SM) [60,61]. It was explicitly shown that a $\mathrm{RG}$ improved L-loop effective potential is consistent when the parameters are calculated using (L+1)-loop RGE's [61].

The observations of Ref. [60,61] were recently applied to the MSSM by Kodaira et al. [58] who extended a previous work of Espinosa and Quiros [62]. Indeed, they find that the two-loop RGM with one-loop EP slightly increase the one-loop RGM result. However, they impose the naive assumption of only one relevant scale $\left(M_{S U S Y}\right)$ below which the SM RGE's are in effect (and the EP contain only a $m_{t}$ correction). Thus, the MSSM parameters are not treated on the same footing as the SM ones, and one expects some modification of their numerical results. However, we note that the correction is positive: recall that the difference between the two RGM approximations is the next-to-leading logarithms, which we found to be positive by examining the scale dependence of the result.

Hempfling and Hoang [59] explicitly calculate the two-loop RGM correction (using the methods of Ref. [47]), finding

$$
\delta \Delta_{h^{0}}^{2} \approx \frac{3}{\left(16 \pi^{2}\right)^{2}} 8 \sqrt{2} G_{\mu} m_{t}^{4} \ln \frac{M_{S U S Y}^{2}}{m_{t}^{2}}\left[\frac{16}{3} g_{s}^{2}-5 h_{t}^{2}\right],
$$

which roughly agrees with our Eq. (C1). 
Those authors go further to derive a diagramatic two-loop result (in a simplified model) which diminishes $\Delta_{h^{0}}$ below its two-loop RGM prediction. They then show that, e.g., if the difference between the pole and running t-quark mass is accounted for in the RGM calculation by appropriate matching conditions, the two methods agree. This again stresses the need for adequate choice of matching conditions. However, it is not clear that there are no other effects of that order of magnitude that can slightly increase $\Delta_{h^{0}}$. [Also, we showed that scalar mixings which are neglected in the above two-loop calculations and in our Eq. (C1), are not apriori negligible.]

In short, all analyses support the conclusion that two-loop logarithms will diminish the one-loop leading logarithm result (in particular, if one properly distinguishes the $t$-quark pole mass from the running mass). However, it is not clear that the prediction of a complete two-loop analysis lies as low as suggested in Ref. [59] (or by our Fig. 10). (If indeed this is the case our upper bounds will be significantly strengthened.)

\section{APPENDIX D: EXTENDED HIGGS SECTORS}

If there is an extended heavy Higgs sector (as is assumed by some authors when trying to explain the light fermion spectrum) with large representations coupling to the third family (i.e., there is no flavor symmetry that forbids such couplings) then (2) does not hold and our constraints are evaded. Alternatively, some models extend the light Higgs sector by adding a SM singlet superfield, $S$, to the spectrum. This is the only addition to the Higgs sector which is consistent (i.e., without fine-tuned cancellations) with coupling constant unification.

The new singlet(s) would mix with the other neutral Higgs bosons and would thus contribute to $m_{h^{0}}^{T}$; e.g., [63],

$$
m_{h^{0}}^{T^{2}} \leq M_{Z}^{2} \cos ^{2} 2 \beta+\lambda_{s}^{2} \frac{\nu^{2}}{2} \sin ^{2} 2 \beta
$$

where $\lambda_{s}\left(\kappa_{s}\right)$ is a new Yukawa coupling in the superpotential: $\lambda_{s} H_{1} H_{2} S\left(\kappa_{s} S^{3}\right)$. (We do not distinguish here our notation of superfields from that of their scalar components.) The MSSM limit with $m_{h^{0}}^{T}=0$ is recovered if $\lambda_{s} \rightarrow 0$, which is indeed the case if $h_{t} \approx h_{t}^{\text {fixed }}$ [64]. However, the new Yukawa couplings (i.e., $\lambda_{s} \neq 0$ ) enable one to realize (2) for somewhat

smaller values of $h_{t}<h_{t}^{\text {fixed }}$ (and $\tan \beta>1$ ). The shift in the allowed region is shown in Fig. 11 for $\lambda_{s}=0.5$ and $\kappa_{s}=0$. Thus, $m_{h^{0}}^{T}$ can be large and $m_{h^{0}}$ can be heavy $\sim 150 \mathrm{GeV}$ $[19,20,64]$ when loop corrections are added. (See also Ref. [65].) 


\section{REFERENCES}

[1] H. Georgi and S. L. Glashow, Phys. Rev. Lett. 32, 438 (1974); H. Georgi, H. R. Quinn, and S. Weinberg, Phys. Rev. Lett. 33451 (1974); E. Witten, Nucl. Phys. B188, 513 (1981); S. Dimopoulos and H. Georgi, ibid. 193, 150 (1981); N. Sakai, Z. Phys. C 11, 153 (1981); S. Dimopoulos, S. Raby, and F. Wilczek, Phys. Rev. D 24, 1681 (1981); W. J. Marciano and G. Senjanovic, ibid. 25, 3092 (1982); L. E. Ibanez and G. G. Ross, Phys. Lett. 105B, 439 (1982); M. B. Einhorn and D. R. T. Jones, Nucl. Phys. B196, 475 (1982).

[2] For reviews, see P. Langacker, Phys. Rep. 72, 185 (1981); in Proceedings of the Ninth Workshop on Grand Unification, Aix les Bains, France, 1988, ed. R. Barloutaud (World Scientific, Singapore, 1988) p.3; H. P. Nilles, Phys. Rep. 110, 1 (1984); in Testing The Standard Model, ed. M. Cvetič and P. Langacker (World Scientific, Singapore, 1991) p. 633; H. E. Haber and G. L. Kane, Phys. Rep. 117, 75 (1985). See also P. Nath et al., Applied N=1 Supergravity (World Scientific, Singapore, 1984); G. G. Ross, Grand Unified Theories (Benjamin, New York, 1984); R. N. Mohapatra, Unification and Supersymmetry (Springer, New York, 1986, 1992). For recent reviews, see, for example, H. E. Haber, Santa Cruz Report No. SCIPP 92/33 (1993); J. L. Lopez, Texas A\&M Report No. CTP-TAMU-42/93 (1993); R. Arnowitt and P. Nath, Texas A\&M Report No. CTP-TAMU-52/93 (1993).

[3] M. S. Chanowitz, J. Ellis, and M. Gaillard, Nucl. Phys. B128, 506 (1977); A. J. Buras, J. Ellis, M. K. Gaillard, and D. V. Nanopoulos, ibid. 135, 66 (1978).

[4] P. Langacker and M. Luo, Phys. Rev. D 44, 817 (1991); J. Ellis, S. Kelley and D. V. Nanopoulos, Phys. Lett. B249, 441 (1990); U. Amaldi, W. de Boer and H. Fürstenau, ibid. 260, 447 (1991); F. Anselmo, L. Cifarelli, A. Peterman and A. Zichichi, Nouvo Cimento 104A, 1817 (1991).

[5] B. Ananthanarayan, G. Lazarides and Q. Shafi, Phys. Rev. D 44, 1613 (1991); M. Bando, T. Kugo, N. Maekawa and H. Nakano, Mod. Phys. Lett. A 7, 3379 (1992); H. Arason et al., Phys. Rev. Lett. 67, 2933 (1991); A. Giveon, L. J. Hall and U. Sarid, Phys. Lett. B271, 138 (1991); S. Kelley, J. L. Lopez, and D. V. Nanopoulos, ibid. 274, 387 (1992).

[6] P. Langacker and N. Polonsky, Phys. Rev. D 47, 4028 (1993).

[7] G. Degrassi, S. Fanchiotti, and A. Sirlin, Nucl. Phys. B351, 49 (1991).

[8] P. Langacker and N. Polonsky, Phys. Rev. D 49, 1454 (1994).

[9] M. Dine, R. Leigh, and A. Kagan, Phys. Rev. D 48, 4269 (1993); Y. Nir and N. Seiberg, Phys. Lett. B309, 337 (1993); P. Pouliot and N. Seiberg, ibid. 318, 169 (1993).

[10] V. Barger, M. S. Berger, and P. Ohmann, Phys. Rev. D 47, 1093 (1993).

[11] M. Carena, S. Pokorski, and C. E. M. Wagner, Nucl. Phys. B406, 59 (1993); W. A. Bardeen, M. Carena, S. Pokorski, and C. E. M. Wagner, Max Planck Report No. MPIPH-93-58 (1993).

[12] V. Barger, M. S. Berger, P. Ohmann, and R. J. Phillips, Phys. Lett. B314, 351 (1993).

[13] L. J. Hall, R. Rattazzi, and U. Sarid, LBL Report No. LBL-33997 (1993).

[14] G. L. Kane, C. Kolda, L. Roszkowski, and J. D. Wells, private communications and Michigan Report No. UM-TH-93-24 (1993).

[15] N. Polonsky, in proceedings of the International Workshop on Supersymmetry and Unification of Fundamental Interactions, Boston, MA, 1993, ed. P. Nath (World Scientific, 
Singapore, 1993) p. 398.

[16] N. Polonsky, Pennsylvania Report No. UPR-0588T (1993).

[17] P. Langacker, in Recent Directions in Particle Theory, ed. J. Harvey and J. Polchinski (World Scientific, Singapore, 1993) p. 141.

[18] For reviews, see M. Sher, Phys. Rep. 179, 273 (1989); J. F. Gunion, H. E. Haber, G. Kane, and S. Dawson, The Higgs Hunter's Guide (Addison-Wesley, Reading, 1990).

[19] J. R. Espinosa and M. Quiros, Phys. Lett. B302, 51 (1993).

[20] G. L. Kane, C. Kolda, and J. D. Wells, Phys. Rev. Lett. 70, 2686 (1993).

[21] M. Bando, T. Kugo, N. Maekawa and H. Nakano, Mod. Phys. Lett. A 7, 3379 (1992);

M. Bando, N. Maekawa, H. Nakano, and J. Sato, ibid. 8, 2729 (1993).

[22] See, for example, J. E. Björkman and D. R. T. Jones, Nucl. Phys. B259 533 (1985); N. K. Flack, Z. Phys. C 30, 247 (1986); M. Drees and M. M. Nojiri, Nucl. Phys. B369, 54 (1992). See also Ref. [10,38,57,47], as well as D. J. Castano, E. J. Piard, and P. Ramond, Florida Report No. UFIFT-HEP-93-18.

[23] K. Inoue, A. Kakuto, H. Komatsu, and S. Takeshita, Prog. Theor. Phys. 67, 1889 (1982).

[24] S. Coleman and E. Weinberg, Phys. Rev. D 7, 1888 (1973); S. Weinberg, ibid. 2887 (1973).

[25] R. Arnowitt and P. Nath, Phys. Rev. D 46, 3981 (1992).

[26] S. Weinberg, Phys. Rev. D 19, 1277 (1979); L. Susskind, ibid. 20, 2619 (1979); P. Sikivie et al., Nucl. Phys. B173, 189 (1980).

[27] R. Barbieri and L. Maiani, Nucl. Phys. B244, 32 (1983).

[28] H. E. Haber and A. Pomarol, Phys. Lett. B302, 435 (1993).

[29] M. Olechowski and S. Pokorski, Phys. Lett. B214, 393 (1988); Nucl. Phys. B404, 590 (1993).

[30] B. Ananthanarayan, G. Lazarides, and Q. Shafi, Phys. Lett. B300, 245 (1993); B. Ananthanarayan and Q. Shafi, Bartol Report No. BA-93-25 (1993).

[31] M. Carena, M. Olechowski, S. Pokorski, and C. E. M. Wagner, CERN Report No. CERN-TH-7163-94 (1994).

[32] A. E. Nelson and L. Randall, Phys. Lett. B316, 516 (1993).

[33] M. Boulware and D. Finnel, Phys. Rev. D 442054 (1991).

[34] C. T. Hill, Phys. Rev. D 24, 691 (1981). See also M. Cvetič and C. R. Preitschopf, Nucl. Phys. B272, 490 (1986).

[35] E. Franco and A. Morelli, Nouvo Cimento 96A, 257 (1986).

[36] M. A. Diaz and H. E. Haber, Phys. Rev. D 46, 3086 (1992).

[37] M. Carena, M. Olechowski, S. Pokorski, and C. E. M. Wagner, CERN Report No. CERN-TH-7060-93 (1993).

[38] V. Barger, M. S. Berger, and P. Ohmann, Madison Report No. MAD/PH/801 (1993).

[39] Similar issues were also studied by B. Ananthanarayan, K. S. Babu, and Q. Shafi, private communications. [After completion of this work we received their paper B. Ananthanarayan et al., Bartol Report No. BA-94-03 (1994).]

[40] We thank J. Erler for focusing our attention on that point.

[41] T. Kon and T. Nonaka, Seikei Report No. ITP-SU-93/05 (1993), and references therein.

[42] J. F. Gunion and H. Pois, UC Davis Report No. UCD-94-01 (1994).

[43] M. Drees, M. Glück, and K. Grassie, Phys. Lett. 157B, 164 (1985). 
[44] J. L. Lopez and D. V. Nanopoulos, Phys. Lett. B266, 397 (1991).

[45] J. Ellis, G. Ridolfi, and F. Zwirner, Phys. Lett B257, 83 (1991); ibid. 262, 477 (1991).

[46] A. Brignole, Phys. Lett. B281, 284 (1992).

[47] H. E. Haber and R. Hempfling, Phys. Rev. D 48, 4280 (1993).

[48] R. Hempfling, DESY Report No. DESY-93-012 (1993).

[49] G. Gamberini, G. Ridolfi, and F. Zwirner, Nucl. Phys. B331, 331 (1990).

[50] M. Claudson, L. J. Hall, and I. Hinchliffe, Nucl. Phys. B228, 501 (1983);

[51] A. Stange, W. Marciano, and S. Willenbrock, Phys. Rev. D 49, 1354 (1994).

[52] G. F. Giudice and E. Roulet, Phys. Lett. B315, 107 (1993), and references therein.

[53] J. F. Gunion, H. E. Haber, and M. Sher, Nucl. Phys. B306, 1 (1988).

[54] J. M. Frere, D. R. T. Jones, and S. Raby, Nucl. Phys. B222, 11 (1983); L. AlvarezGaume, J. Polchinski, and M. B. Wise, ibid. 221, 495 (1983); C. Kounnas, A. B. Lahanas, D. V. Nanopoulos, and M. Quiros, ibid. 236, 438 (1984); J. P. Derendinger and C. A. Savoy, ibid. 237, 307 (1984).

[55] H. Komatsu, Phys. Lett. B215, 323 (1988).

[56] S. Kelley, J. L. Lopez, D. V. Nanopoulos, H. Pois, and K. Yuan, Nucl. Phys. B398, 3 (1993).

[57] P. H. Chankowski, Phys. Rev. D 41, 2877 (1990).

[58] J. Kodaira, Y. Yasui, and K. Sasaki, Hiroshima Report No. HUPD-9316 (1993).

[59] R. Hempfling and A. H. Hoang, DESY Report No. DESY-93-162 (1993).

[60] B. Kastening, Phys. Lett. B283, 287 (1992); C. Ford, D. R. T. Jones, and P. W. Stephenson, Nucl. Phys. B395, 17 (1993).

[61] M. Bando, T. Kugo, N. Maekawa, and H. Nakano, Phys. Lett. B301, 83 (1993).

[62] J. R. Espinosa and M. Quiros, Phys. Lett. B266, 389 (1991).

[63] L. Durand and J. Lopez, Phys. Lett. B217, 463 (1989); M. Drees, Int. J. Mod. Phys. A 4, 3635 (1989).

[64] T. Elliott, S. F. King, and P. L. White, Southampton Report No. SHEP-92-93-21 (1993); and references therein.

[65] After completion of this work we received a paper by B. C. Allanach and S. F. King, Southampton Report No. SHEP-93-94-15 (1994), where similar issues are discussed. 


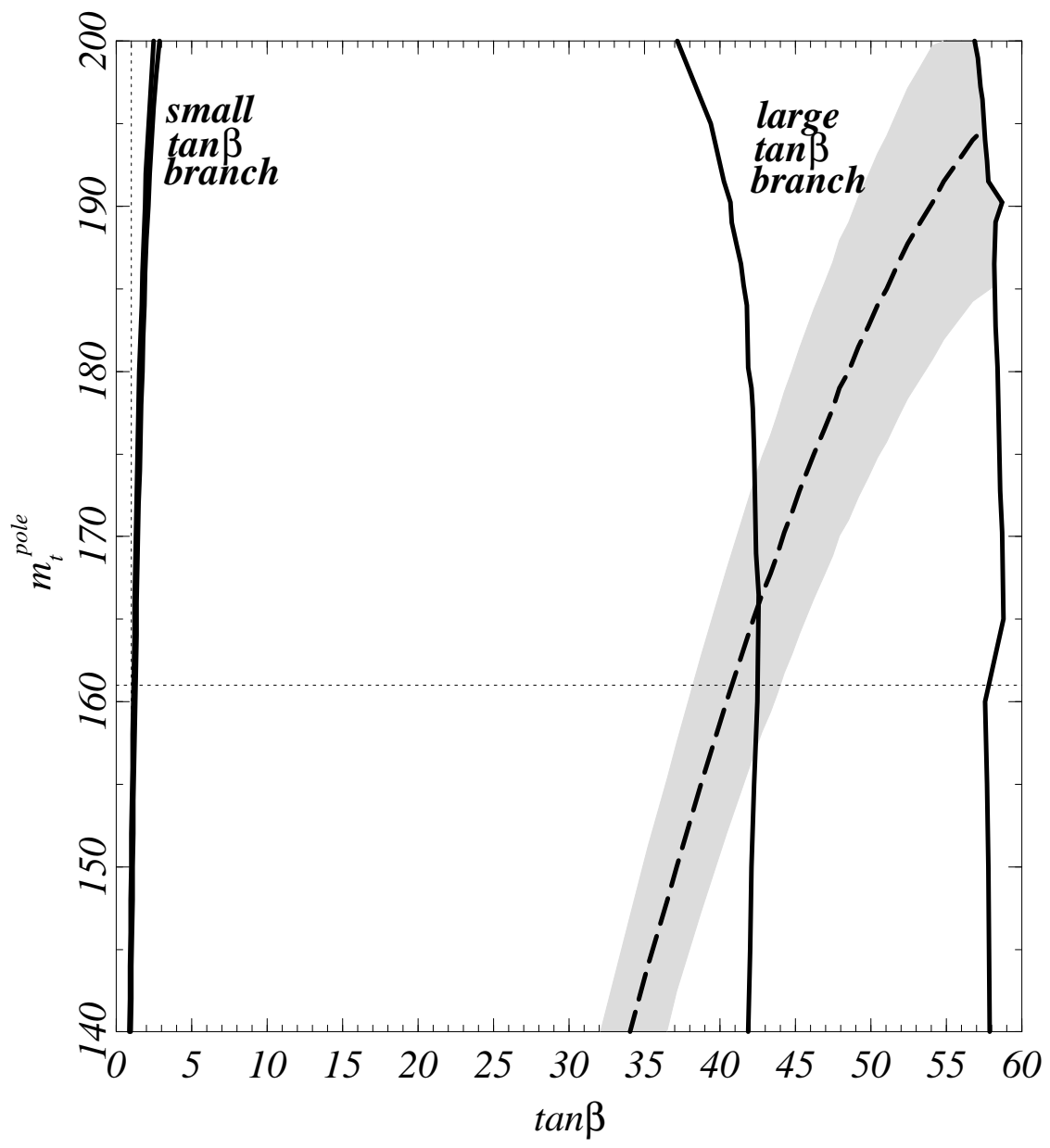

FIG. 1. The $t$-quark pole mass (in $\mathrm{GeV}$ ) - $\tan \beta$ plane is divided into five different regions. Two areas (low- and high-tan $\beta$ branches) are consistent with perturbative two-Yukawa ( $b$ and $\tau$ ) unification and with an upper bound of $\frac{4.45}{0.85} \mathrm{GeV}$ for the $b$-quark current mass. The 0.85 factor takes into account $\mathrm{a} \sim \pm 15 \%$ theoretical uncertainty in the numerical calculation due to the matching conditions. Between the two branches the $b$-quark mass is too high. For a too low (high) $\tan \beta$, the $t$-quark ( $b$-quark) Yukawa coupling diverges. The region where all three (third-family) Yukawa couplings unify (dashed line) intersects the allowed high-tan $\beta$ branch. [The three-Yukawa region is calculated without imposing any constraints on $m_{b}$, and is assigned a $\sim 5 \%$ uncertainty (shaded area) from corrections to the $h_{t} / h_{b}$ ratio.] The $t$-quark mass range suggested by the electroweak data and the $\tan \beta=1$ line are indicated (dotted lines) for comparison. 

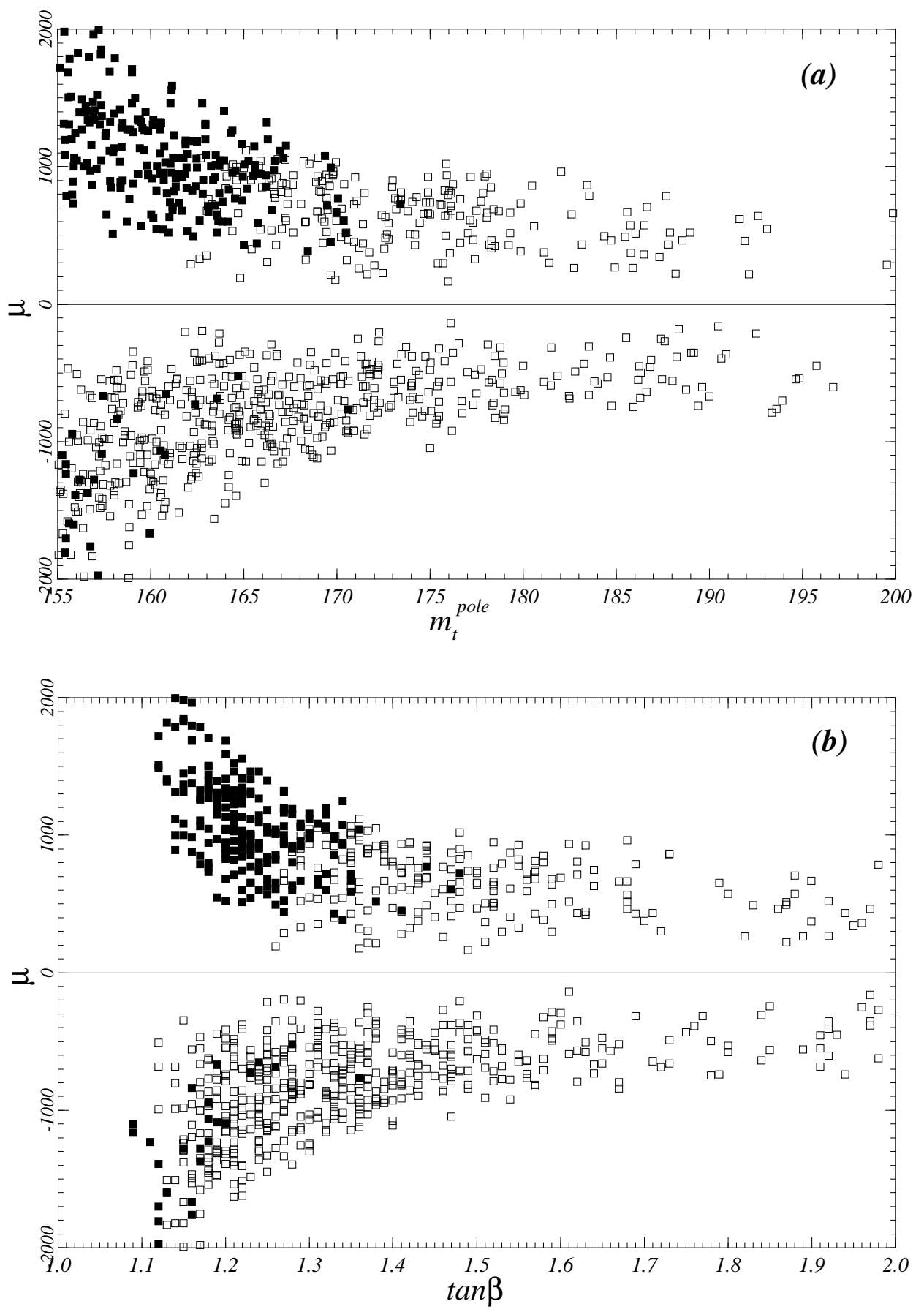

FIG. 2. The prediction for the Higgsino mass parameter $\mu$ (after absorbing $\Delta V$ in the redefined tree-level parameters) as a function of $(a)$ the $t$-quark pole mass and of $(b) \tan \beta$. (The $t$-quark mass and $\tan \beta$ are strongly correlated.) Filled squares indicate (probably unacceptable) points for which the SM minimum is only a local minimum. All masses are in $\mathrm{GeV}$ 


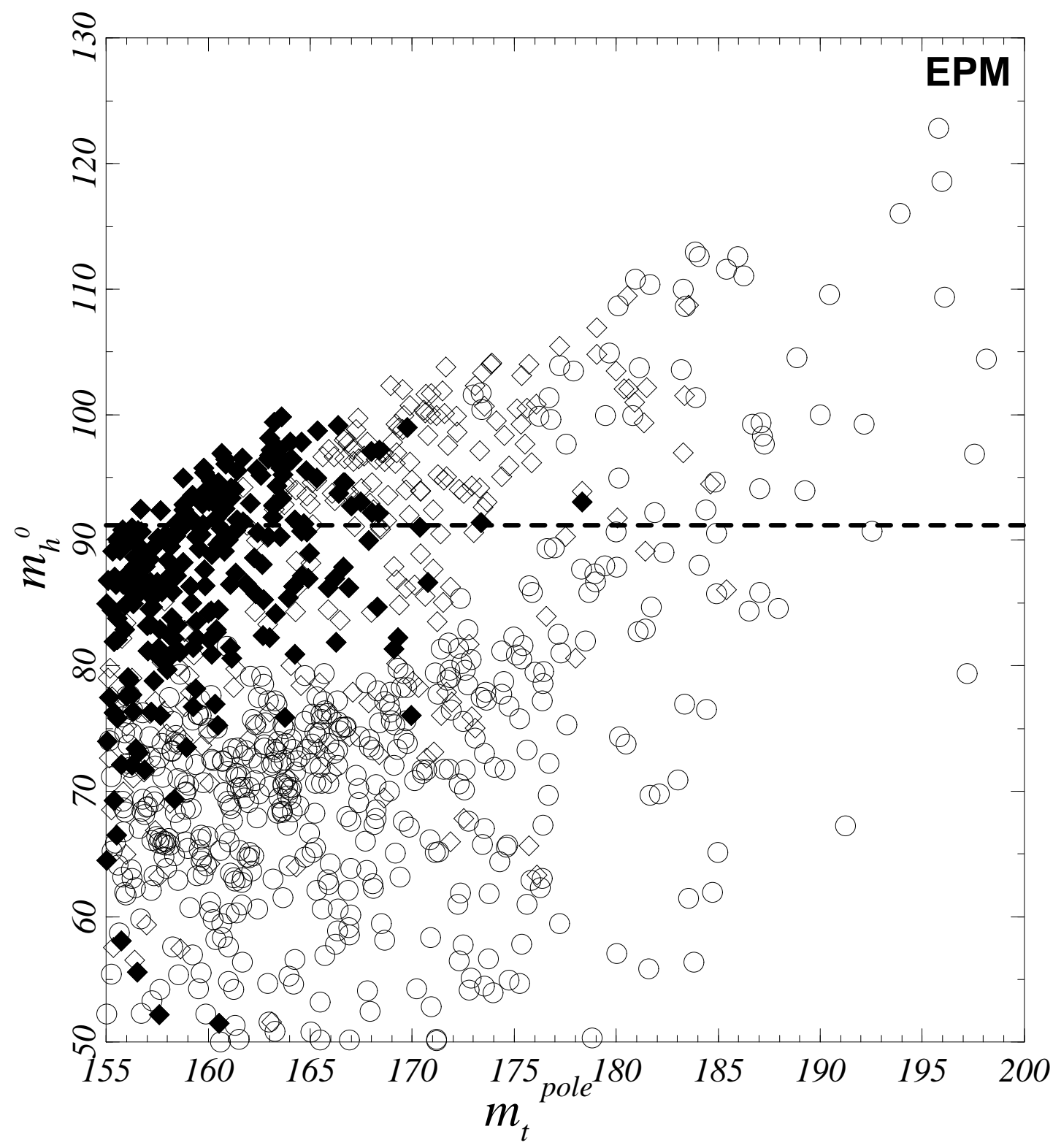

FIG. 3. A scatter plot of the Higgs boson mass (calculated in the EPM with the subtraction scale $Q$ at the $Z$-pole) vs. the $t$-quark pole mass. The $o$ indicate points which have no (potentially dangerous) negative-energy color and/or charge breaking minimum. The $\diamond$ have such a minimum but it is a (safe) local one, i.e., it lies above the standard-model minimum. Points indicated by a filled diamond have an unacceptable CCB global minimum. The $Z$ mass is indicated for comparison (dashed line). All masses are in GeV. 


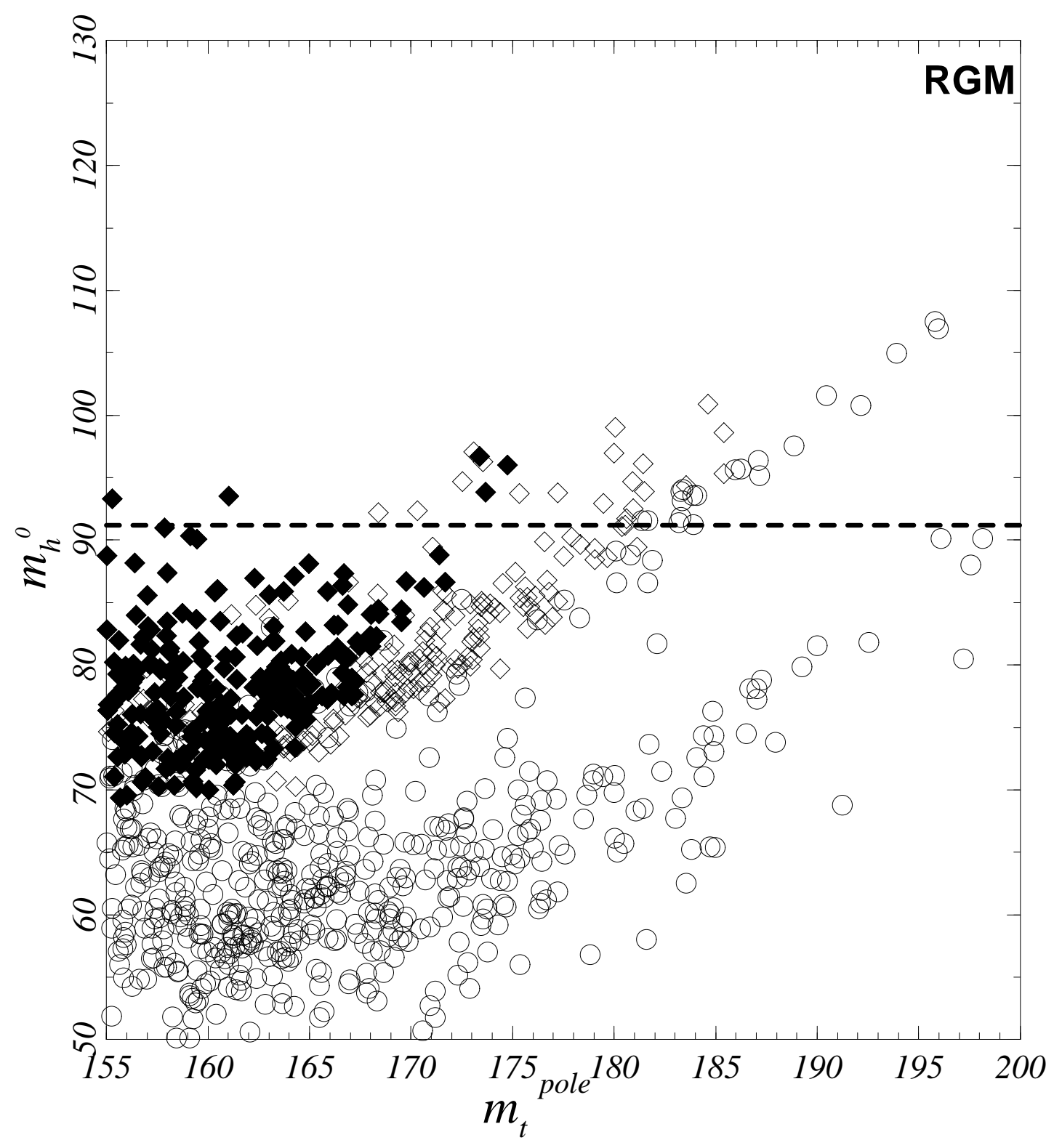

FIG. 4. Same as in Fig. 3 except the mass is calculated using the RGM (numerically). 


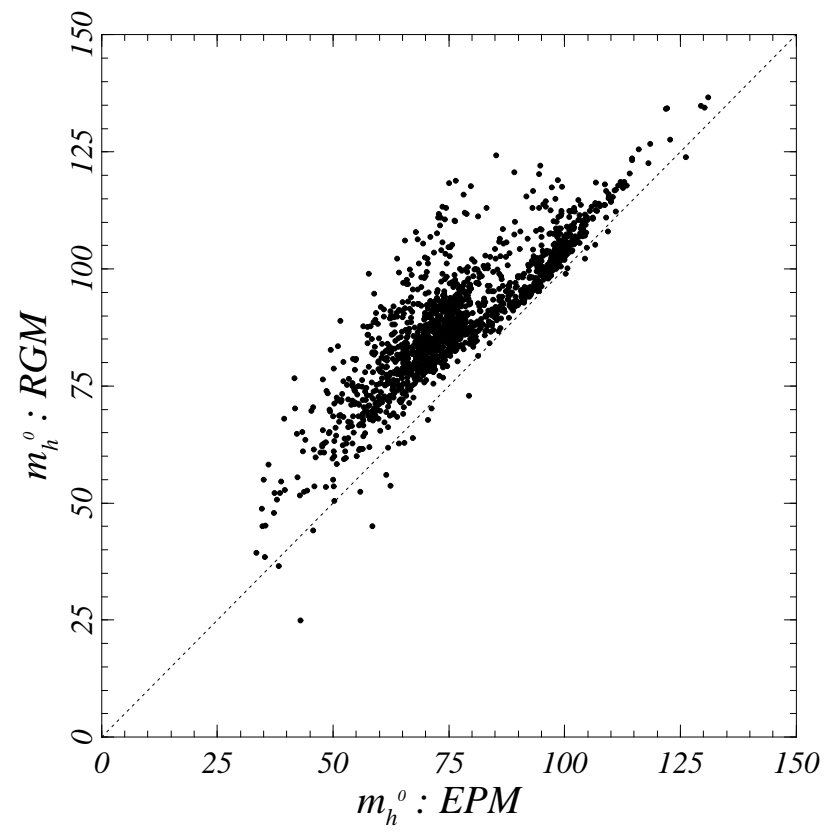

FIG. 5. The EPM (with the subtraction scale $Q$ at the $Z$-pole) calculation of the Higgs boson mass is compared with the mass calculated in the RGM but using the Haber and Hempfling leading logarithm formula. All masses are in GeV. Points corresponding to global GCCB minima (indicated by a filled diamond in Figs. 3 and 4 ) are omitted.

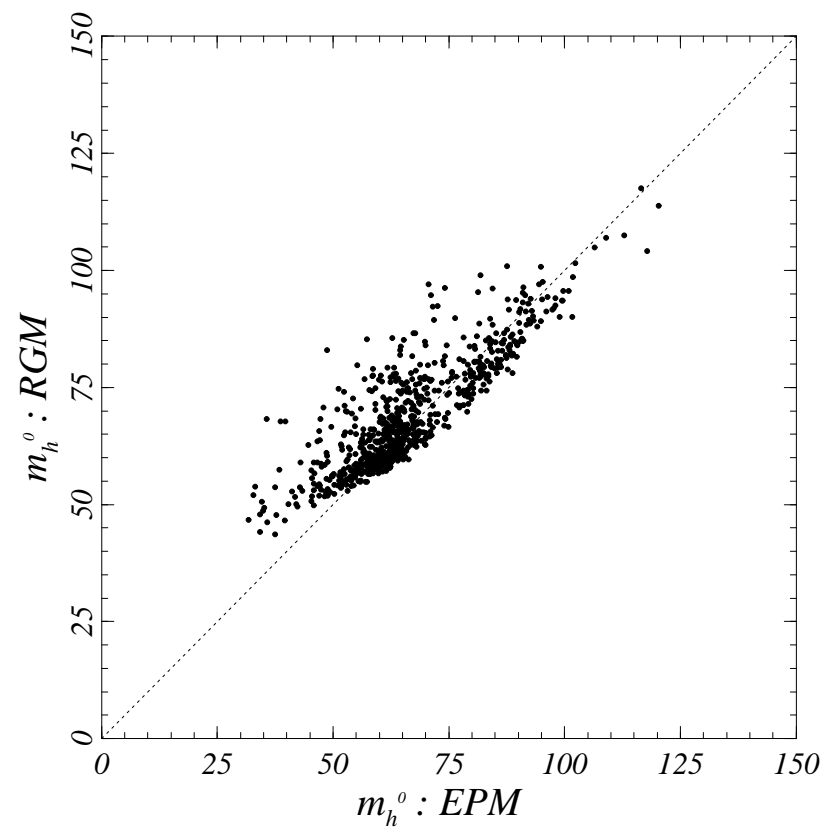

FIG. 6. Same as in Fig. 5, except $Q=600 \mathrm{GeV}$ and the RGM calculation is carried out numerically. 

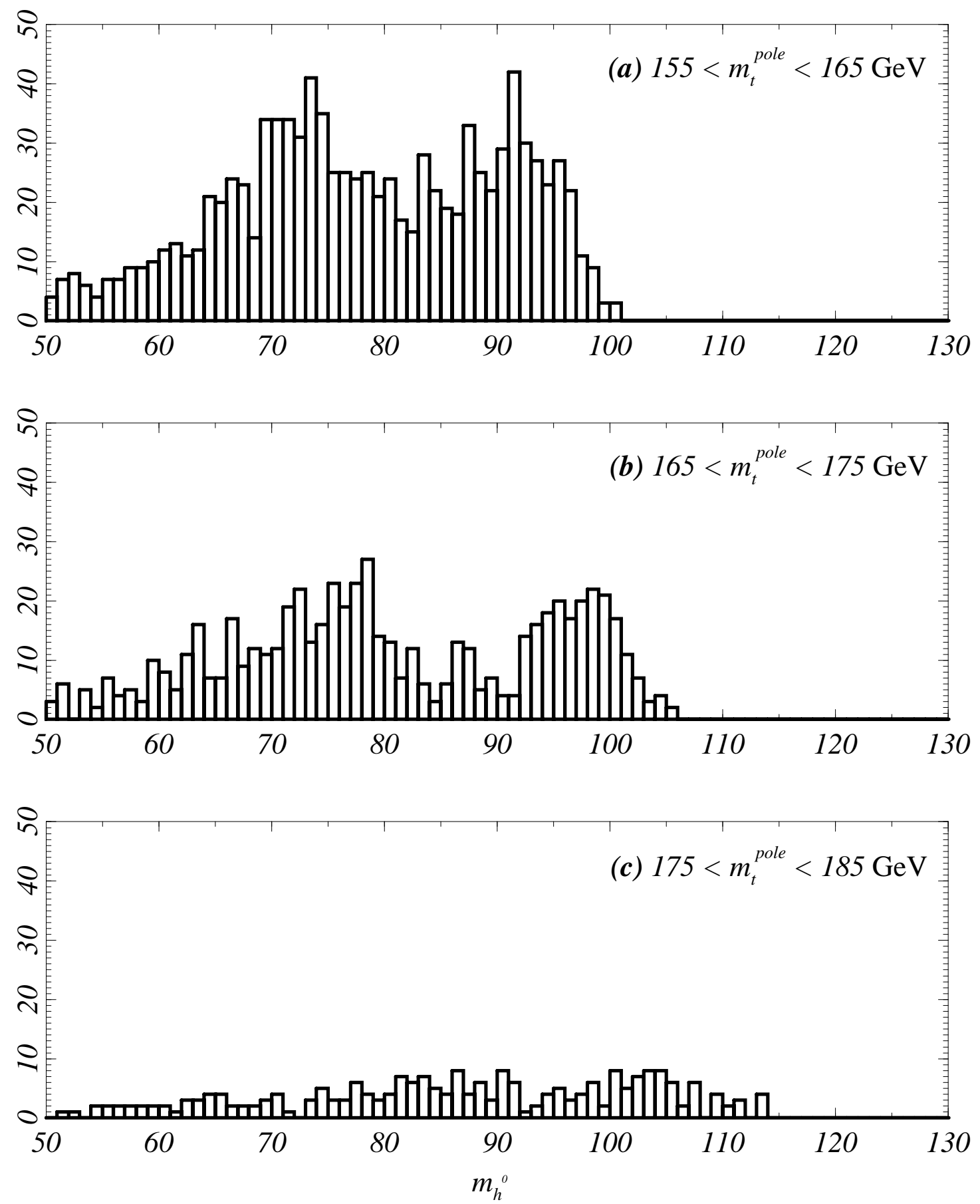

FIG. 7. The Higgs boson mass (in $\mathrm{GeV}$ ) distribution in a sample monte-carlo calculation (using the EPM with the subtraction scale $Q$ at the $Z$-pole) for the $t$-quark pole mass in the range $(a)$ $[155,165],(b)[165,175],(c)[175,185] \mathrm{GeV}$. 

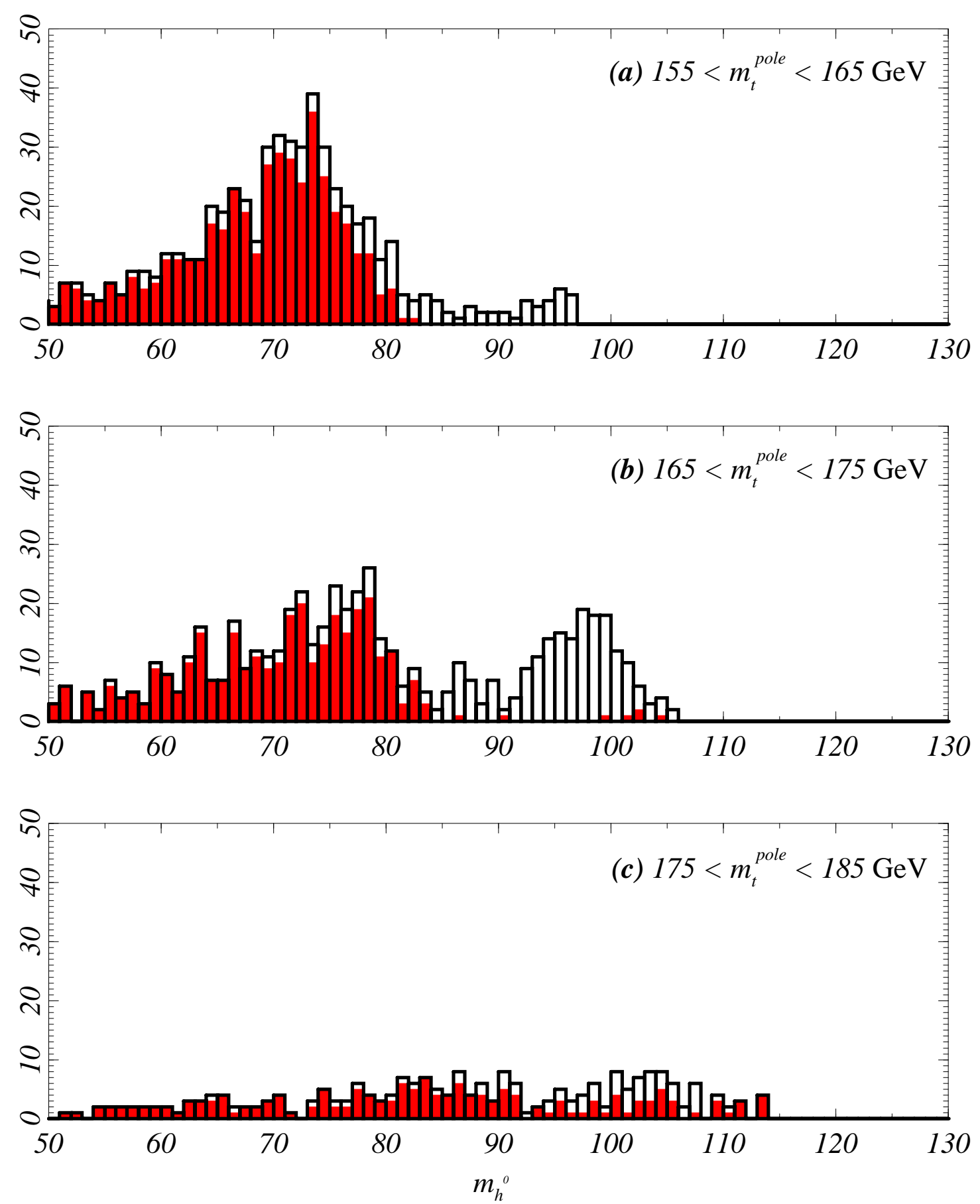

FIG. 8. Same as in Fig. 7 except for the omission of points which correspond to a global color and/or charge breaking minimum (marked by a filled diamond in Fig. 3). For comparison, the shaded areas indicate points which are consistent with the constraint (13) (marked by a circle in Fig. 3). The difference between the two distributions is points with only a local CCB minimum unnecessarily excluded by (13) (marked by open diamonds in Fig. 3). 

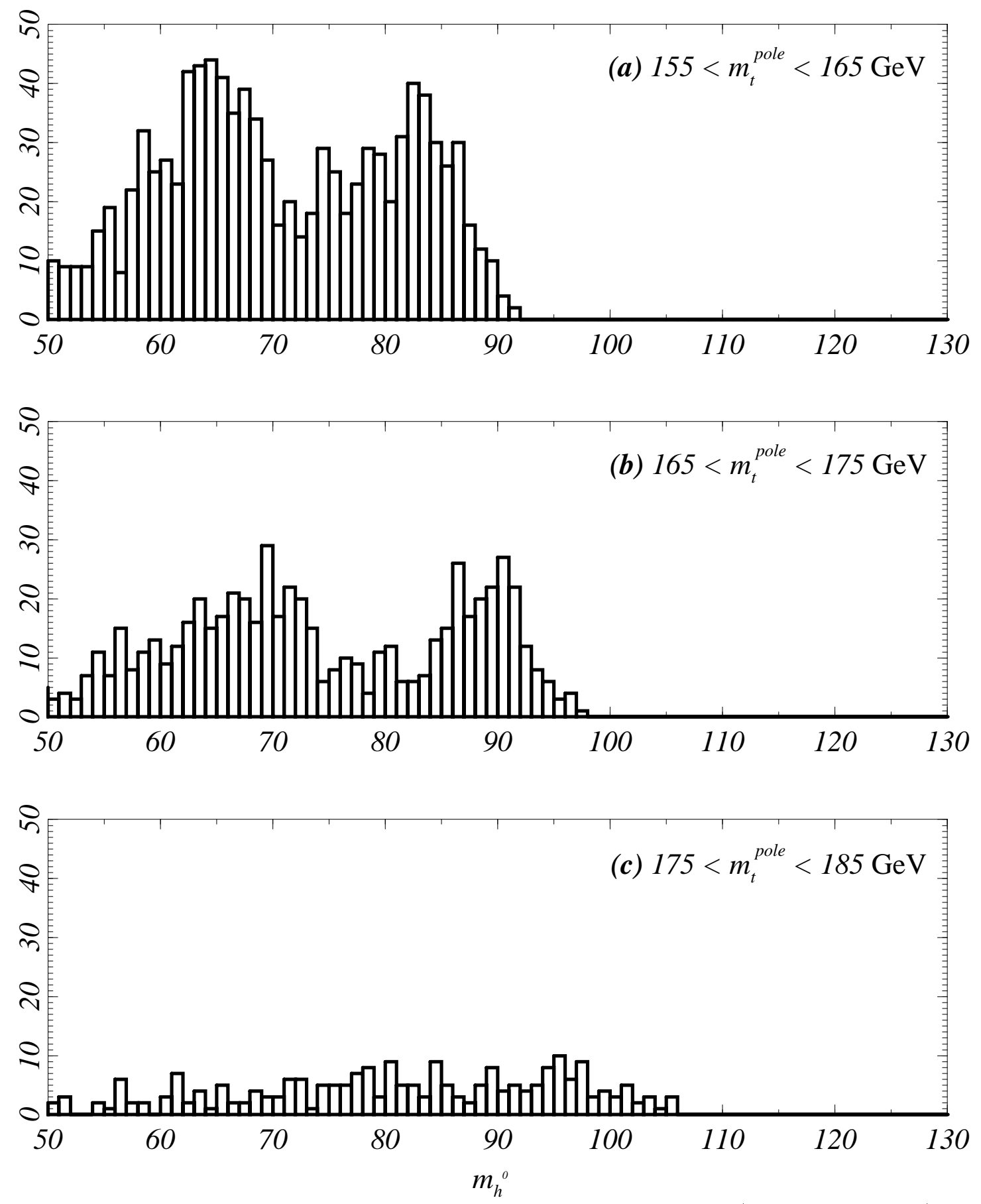

FIG. 9. Same as Fig. 7 except for substituting the $t$-quark running (rather than pole) mass in the mass expressions for the scalars. 

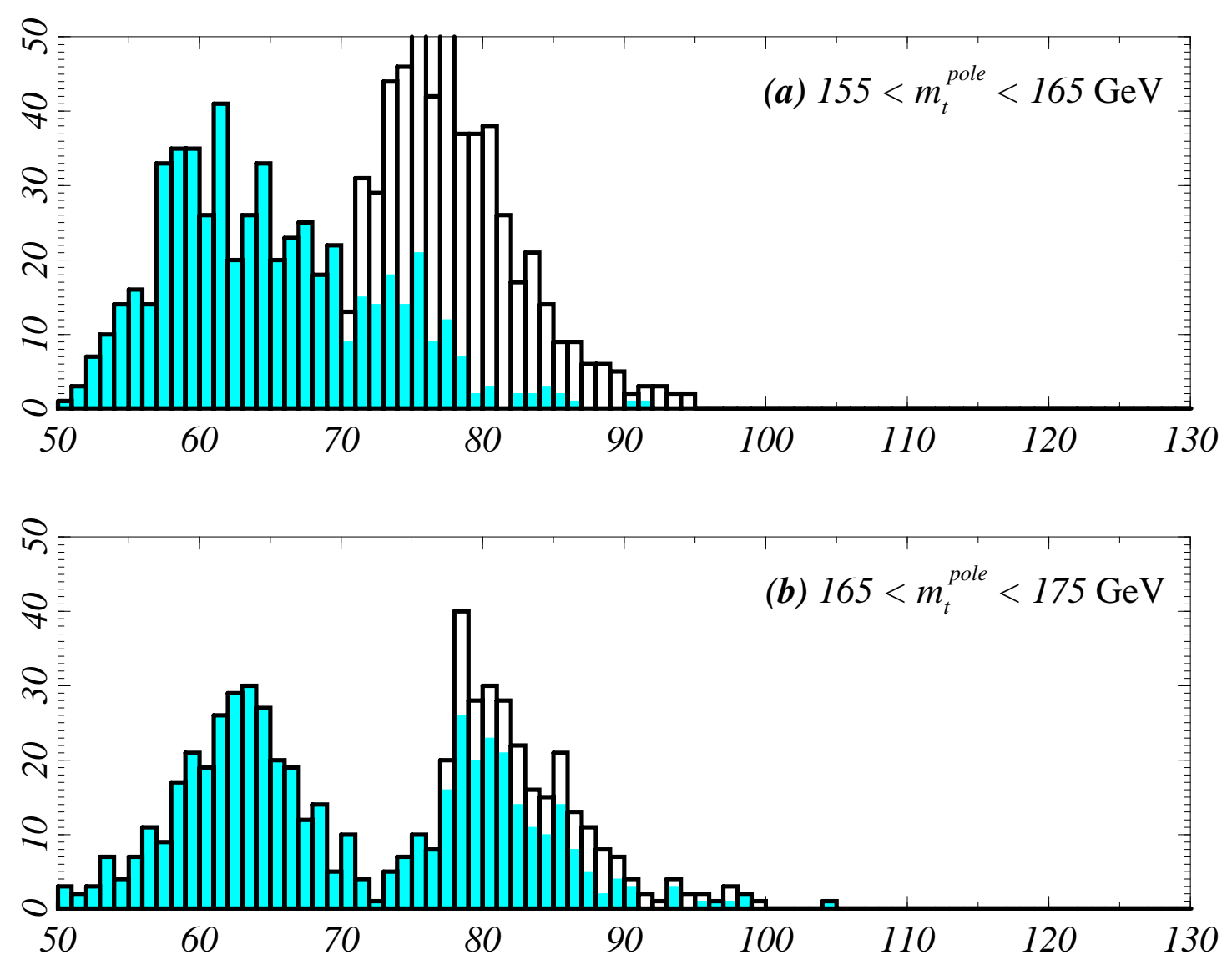

FIG. 10. Same as in Fig. 7a-b except using the RGM (with numerical integration). The distributions omitting points which correspond to a global color and/or charge breaking minimum (marked by a filled diamond in Fig. 4) (lightly shaded areas) are also indicated, and should be compared with the total (shaded and unshaded) areas in Fig. 8. (For a $t$-quark heavier than about 180 GeV the 1HDM assumption that underlies the calculation is not always accurate - see Appendix C.) 


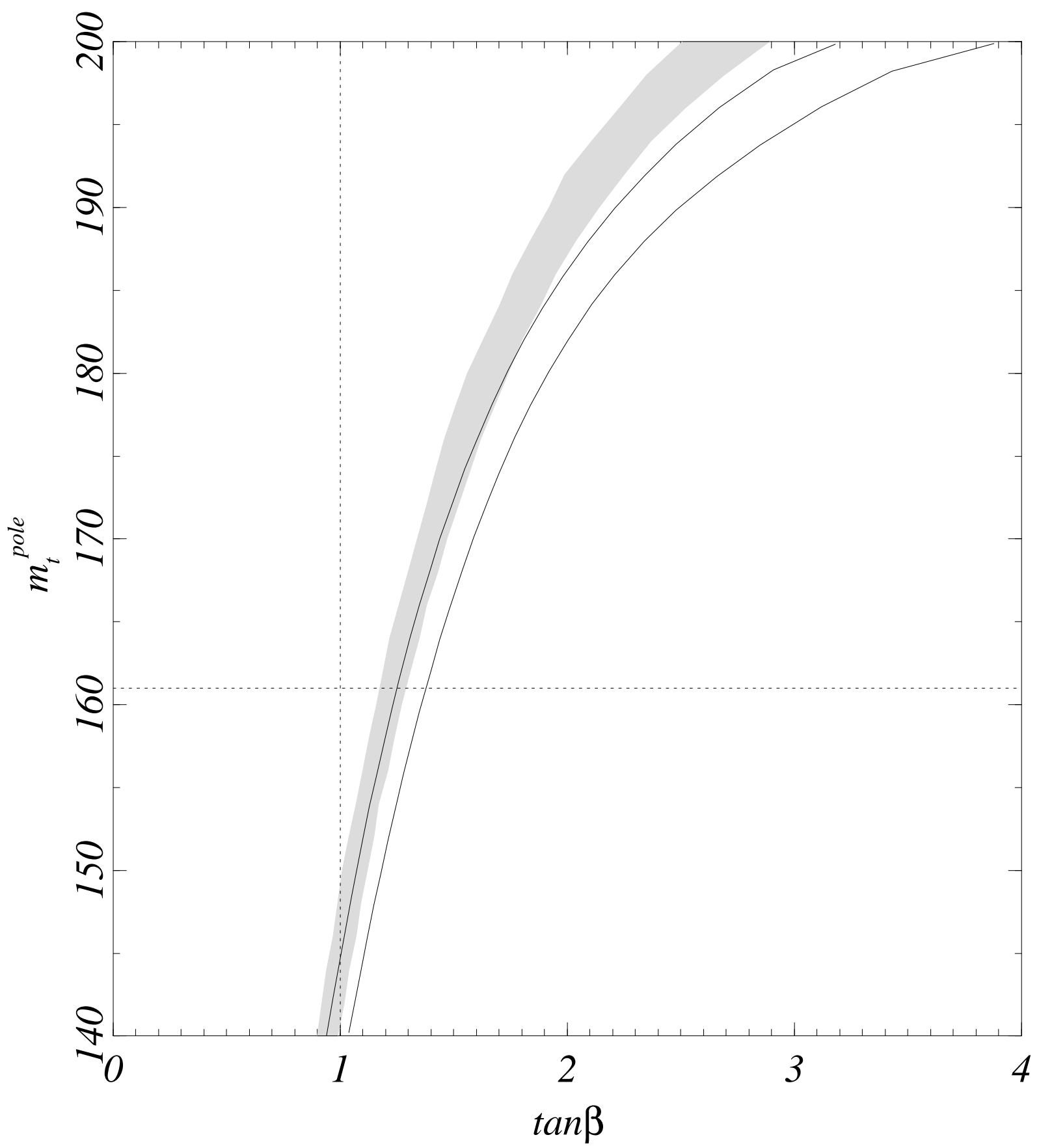

FIG. 11. The low $\tan \beta$ branch in the presence of an additional Higgs field with $\lambda_{s}=0.5$ and $\kappa_{s}=0$. The allowed area when there is no singlet field $\left(\lambda_{s}=0\right)$ is shown for comparison (shaded region). the $t$-quark pole mass (given in $\mathrm{GeV}$ ) range suggested by precision data and $\tan \beta=1$ are indicated (dotted lines). 